\title{
Magnetic hysteresis in two model spin systems
}

\author{
Madan Rao,* H. R. Krishnamurthy, and Rahul Pandit \\ Department of Physics, Indian Institute of Science, Bangalore 560012, India
}

\begin{abstract}
A systematic study of hysteresis in model continuum and lattice spin systems is undertaken by constructing a statistical-mechanical theory wherein spatial fluctuations of the order parameter are incorporated. The theory is used to study the shapes and areas of the hysteresis loops as functions of the amplitude $\left(H_{0}\right)$ and frequency $(\Omega)$ of the magnetic field. The response of the spin systems to a pulsed magnetic field is also studied. The continuum model that we study is a three-dimensional $\left(\Phi^{2}\right)^{2}$ model with $O(N)$ symmetry in the large- $N$ limit. The dynamics of this model are specified by a Langevin equation. We find that the area $A$ of the hysteresis loop scales as $A-H_{0}^{0.66} \Omega^{0.33}$ for low values of the amplitude and frequency of the magnetic field. The hysteretic response of a twodimensional, nearest-neighbor, ferromagnetic Ising model is studied by a Monte Carlo simulation on $10 \times 10,20 \times 20$, and $50 \times 50$ lattices. The framework that we develop is compared with other theories of hysteresis. The relevance of these results to hysteresis in real magnets is discussed.
\end{abstract}

\section{INTRODUCTION}

Nonequilibrium effects associated with first-order phase transitions have been the subject of a fair amount of experimental and theoretical study. Examples include the growth of domains following a quench from a onephase region to a $q$-phase region $(q \geq 2)$-both early stages' (nucleation and spinodal decomposition) and late stages' of growth. However, hysteresis, the most commonly observed nonequilibrium phenomenon at a firstorder phase transition, has not received as much attention. In this paper, we study hysteresis in a variety of spin systems (both continuous and lattice systems).

There is both a formal and an experimental motivation for our study. We discuss both of these below before presenting the principal results of our study.

Consider first the formal motivation. Figure 1 shows a schematic phase diagram of a ferromagnet in the magnetic field $H$ and temperature $T$ plane. With $T$ fixed and below $T_{c}$, let us start with a magnetic field $+H_{0}$ and cycle the field to $-H_{0}$ and back across the first-order

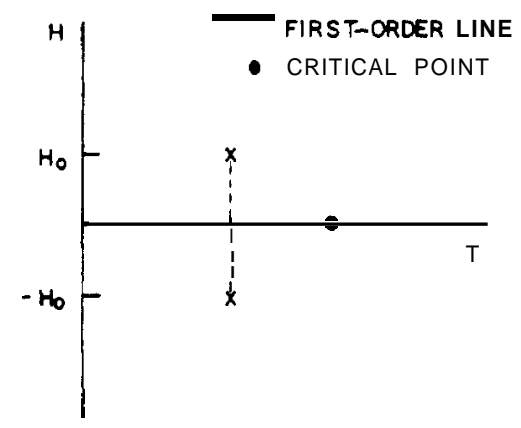

FIG. 1. Schematic phase diagram of a ferromagnet in the $H$ $T$ plane. The magnetic field is changed from $+H_{0}$ to $-H_{0}$ across the $H=0, T<T_{c}$ first-order phase boundary. boundary $\boldsymbol{H}=0, T<T_{c}$. A plot of the magnetization $\mathbf{M}$ (or magnetic induction $\mathbf{B}$ ) versus the field $\mathbf{H}$ yields the familiar hysteresis loop (Fig. 2). We seek the response of a spin system (below its ordering temperature) to a timevarying magnetic field given by $H=H_{0} \sin (\Omega t)$, where $H_{0}$ is the amplitude and $\Omega$ the frequency. In the limit $\Omega \rightarrow 0$, we expect the magnetization $M$ to exhibit a discontinuity at $H=O$ given by $M=M_{\mathrm{eq}} \operatorname{sgn}(H)$ [Fig. 3(a)], where $M_{\mathrm{eq}}$ is the equilibrium magnetization as

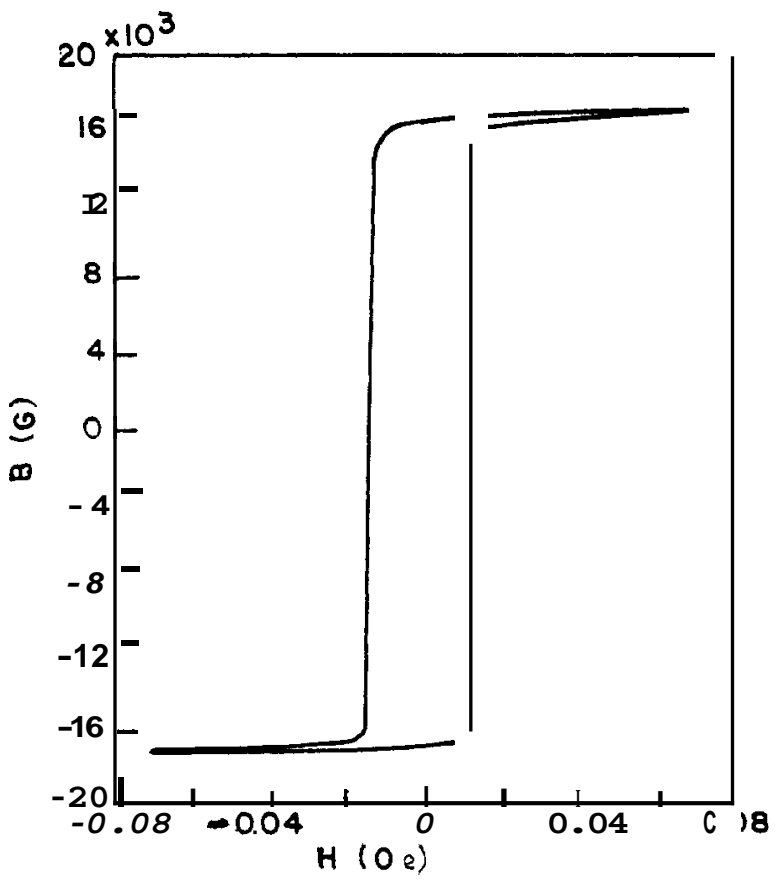

FIG. 2. Hysteresis loop of a single crystal of silicon iron. The $\boldsymbol{B}$ scale is only approximate. 


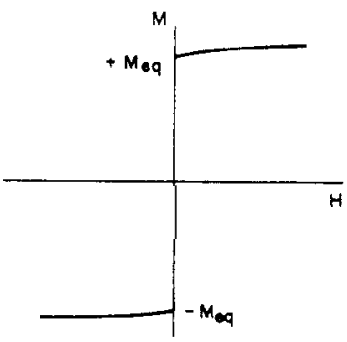

(a)

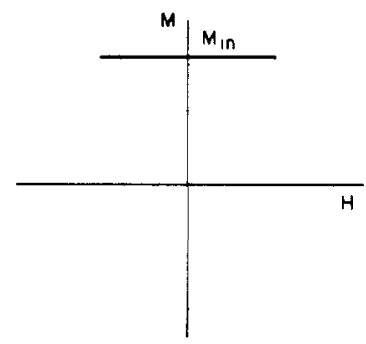

(b)
FIG. 3. (a) When $\Omega=0$, the magnetization jumps from its equilibrium value of $M_{\text {eq }}\left(H_{0}\right)$ to $M_{\text {eq }}\left(-H_{0}\right)$, (b) when $\Omega=\infty$, the magnetization does not respond to the magnetic field and remains at its initial value, $M_{1 n}$.

$H \rightarrow 0+$. In the limit $\Omega \rightarrow \infty$, the spin system cannot respond to the oscillating field, so we expect $M(t)=M_{\text {in }}$ [Fig. 3(b)] for all times. $M_{\text {in }}$ is the initial value of the magnetization of the spin system. The question we seek to answer is the following: How does the discontinuity in $M$ at $H=0$ as $\Omega \rightarrow 0$ evolve into $M(t)=M_{\text {in }}$ as $\Omega \rightarrow \infty$ (Fig. 3)? As $\Omega$ increases slightly away from zero, the discontinuous jump opens out into a loop enclosing a nonzero area. Further, as $\Omega$ decreases slightly away from infinity, the $M(t)=M_{\text {in }}$ line opens out into an ellipse. Does the area of the loop scale as a function of $H$, and $\Omega$ for small and large R? We show that the answer to these questions require a systematic study of the dependence of the shape of the hysteresis loop on the frequency $\Omega$ and the amplitude $H_{0}$ of the magnetic field.

We therefore undertake such an investigation of hysteresis in a variety of model spin systems. This paper contains a detailed analysis of hysteresis in the $N$ component $\left(\boldsymbol{\Phi}^{2}\right)^{2}$ model having $\mathrm{O}(N)$ symmetry in three dimensions. We study this model in the $N \rightarrow \infty$ limit. The dynamics of the order parameter of this model are given by a Langevin equation. We also study hysteresis in a two-dimensional Ising ferromagnet with nearestneighbor interactions. We perform a Monte Carlo simulation using the Metropolis algorithm for flipping spins.

Consider next the experimental motivation: Most experimental studies of hysteresis in magnets concentrate on the dependence of hysteresis loops on anisotropies, impurities, and magnetoelastic couplings, principally with a view to technological applications. However, there have been quite a few studies of the amplitude and frequency dependence of hysteresis loops, ${ }^{3,4}$ some dating as far back as the end of the past century. ${ }^{5-7}$ We summarize the findings of these studies below.

There has been a lot of work on the dependence of the hysteresis loop on the amplitude of the magnetic field. For small fields, the loop does not saturate and appears as an ellipse with sharp tips inclined at an angle to the $H$ axis. An increase in the amplitude of the field $H_{0}$ makes the loop larger and increases its angle of inclination. With a further increase in the field amplitude, the curvature at the tip changes sign showing the beginnings of saturation. For fields greater than or equal to the satura- tion field (which is material and frequency dependent), the loop saturates. This sequence of events is shown in Fig. 4 for a Permalloy magnet.

The area of the hysteresis loop is a measure of the energy dissipated into the system per cycle and is

$$
W_{H}=\oint B d H=\frac{1}{4 \pi} \oint H d M
$$

$W_{H}$ is expressed in erg $/ \mathrm{cm}^{3}$ per cycle when $M$ and $H$ are in $\mathrm{G}$ and Oe, respectively. In Fig. 5 the hysteresis losses are plotted against the maximum induction $B_{m}$ for several specimens. For low values of $B, W_{H} \approx B_{m}^{3}$ (see Sec. IV). For values of $B_{m}$ ranging from 500 to $15000 \mathrm{G}$ in iron, the area of the hysteresis loop is given quite accurately by the Steinmetz law ${ }^{7,5,3}$

$$
W_{H} \approx B_{m}^{1.6}
$$

represented by the dashed line in Fig. 5. It is seen that a wide variety of soft magnets satisfy the Steinmetz law. In some materials, the exponent of $B_{m}$ increases to 1.7 and then to 2 as the value of $B_{m}$ increases. $^{3}$

In contrast to the work done on the amplitude dependence of hysteresis loops, there has not been a systematic study of the frequency dependence of hysteresis loops. In fact, there exists no extensive experimental survey of the nature of hysteresis loops as a function of both the amplitude and frequency of the applied field. However, careful studies of the frequency dependence of hysteresis loops in ferrites have been made. ${ }^{4}$ In the early works of Ewing and co-workers, ${ }^{5.6}$ there are discussions of the frequency dependence of hysteresis loops in soft iron. These studies suggest that the frequency of the magnetic field affects

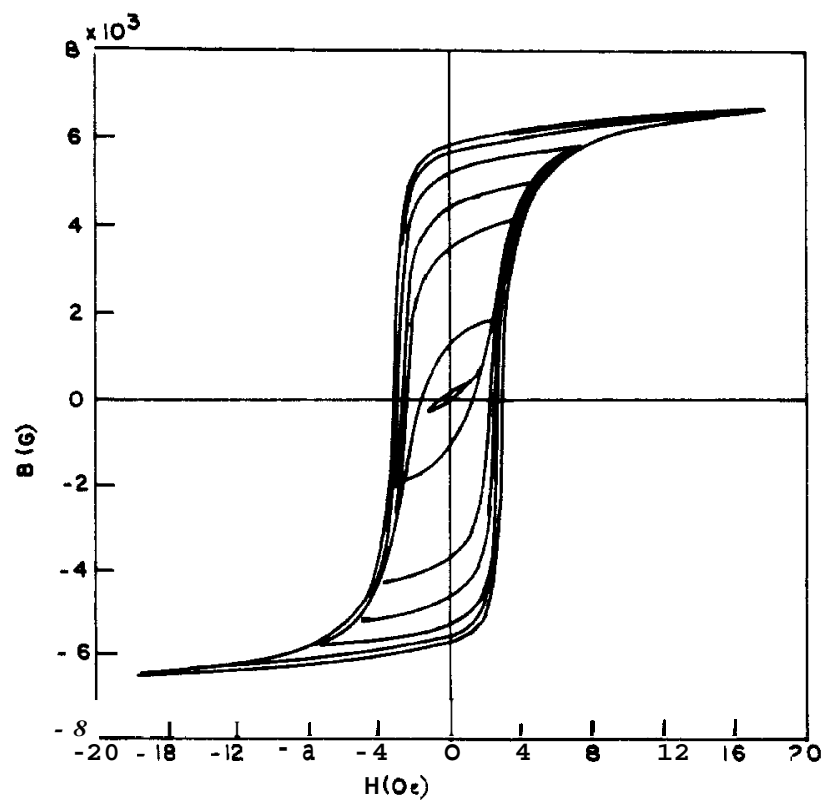

FIG. 4. Family of hysteresis loops for various field strengths in 4-79 Permalloy. The inner loops, which do not show signs of saturation are called minor loops (after Bozorth, 1956, Ref. 3). 


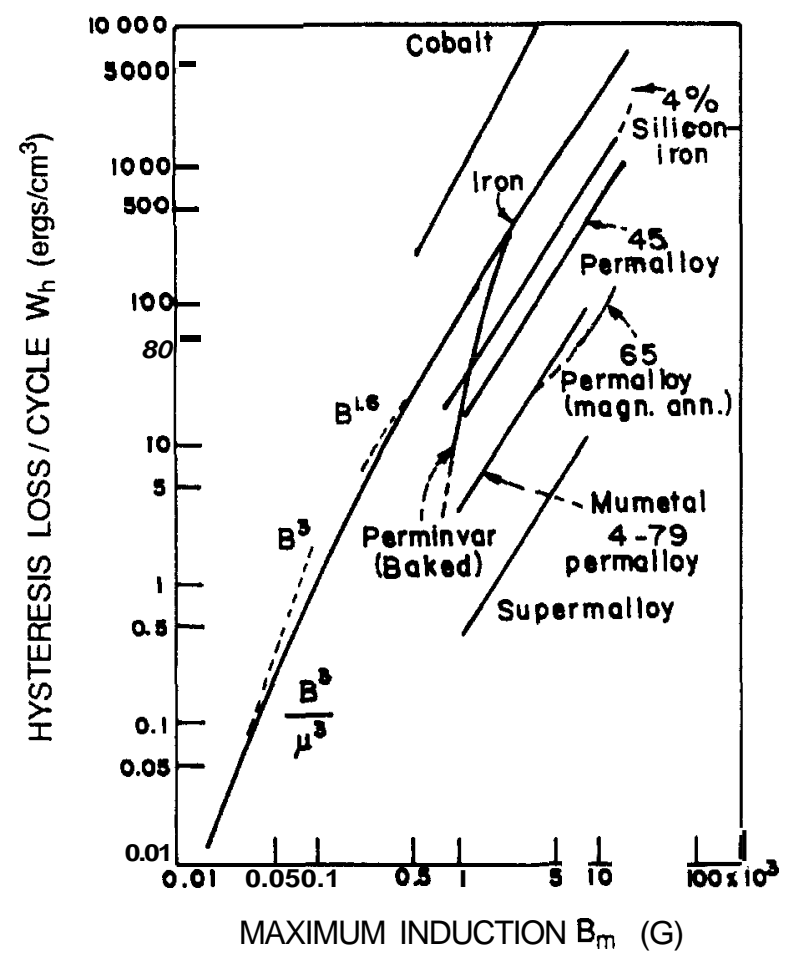

FIG. 5. The energy dissipated per cycle as a function of the maximum induction for several magnets. For small inductions, the energy dissipation goes as $\boldsymbol{B}_{m}^{3}$ (Rayleigh law) as shown by the dotted line. For larger values of $B_{m}$, the Steinmetz law holds for a wide variety of soft magnets (after Bozorth, 1956, Ref. 3).

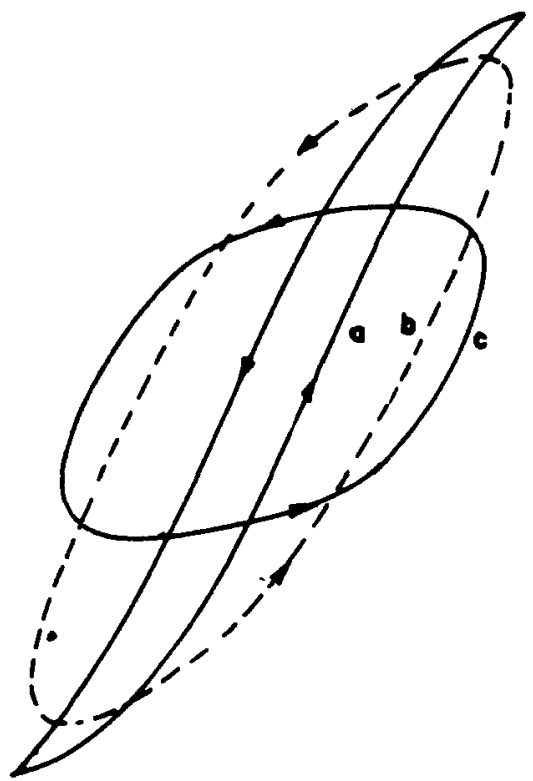

FIG. 6. Hysteresis loops obtained on a magnetic-curve tracer for soft iron bars for various frequencies of the applied field: (a) cycle performed slowly, (b) period of the cycle $=3 \mathrm{sec}$, (c) period of the cycle $=0.43 \mathrm{sec}$ (after Ewing and Klassen, 1893, Ref. 5). the hysteresis loops only for low fields. At high fields no effect of the frequency was noticed. Figure 6 shows the hysteresis loops of a soft iron bar when (a) the cycle was performed very slowly, (b) the period was $3 \mathrm{sec}$, and (c) the period was $\mathbf{0 . 4 3} \mathrm{sec}$. In these experiments the amplitude of the field was held fixed. It was also found that the area of the curve increased at first and finally decreased as the frequency was increased.

As mentioned before, there have been fairly extensive studies of the frequency dependence of hysteresis loops in ferrites. ${ }^{4}$ It is found that ferrites with a high initial permeability $\left(\mu_{0}>400\right)$ have frequency-dependent magnetization curves for frequencies below the ferromagneticresonance (FMR) frequency. Figure 7 shows data for a manganese ferrous ferrite $\left(\mu_{0}=860\right.$, FMR frequency $\approx 5$ $\mathrm{MHz}$ ).

The distortion of a core material is defined as the ratio of the amplitudes of the third harmonic $V_{3}$ and the fundamental $V_{1}$ of the secondary voltage of an open transformer completely filled with this core material, when a sinusoidally varying voltage is applied across the primary coil. $^{4}$ The distortion measures roughly the deviation of the hysteresis loop from ellipticity. Figure 8 gives, for the manganese ferrous ferrite of Fig. 7, the results of the distortion measurements at four frequencies. It is seen that this quantity depends very much on frequency, being almost absent at $\mathbf{7 0 0} \mathrm{kHz}$. Thus, the shape of the hysteresis loop for this ferrite must likewise change with frequency: at low frequencies it is the well-known loop with sharp tips, whereas at high frequencies $\boldsymbol{B}$ is a linear function of $\boldsymbol{H}$, but phase shifted, giving rise to an elliptical loop.

Insulating magnets have a different frequency response from that of metallic magnets since there are eddy-

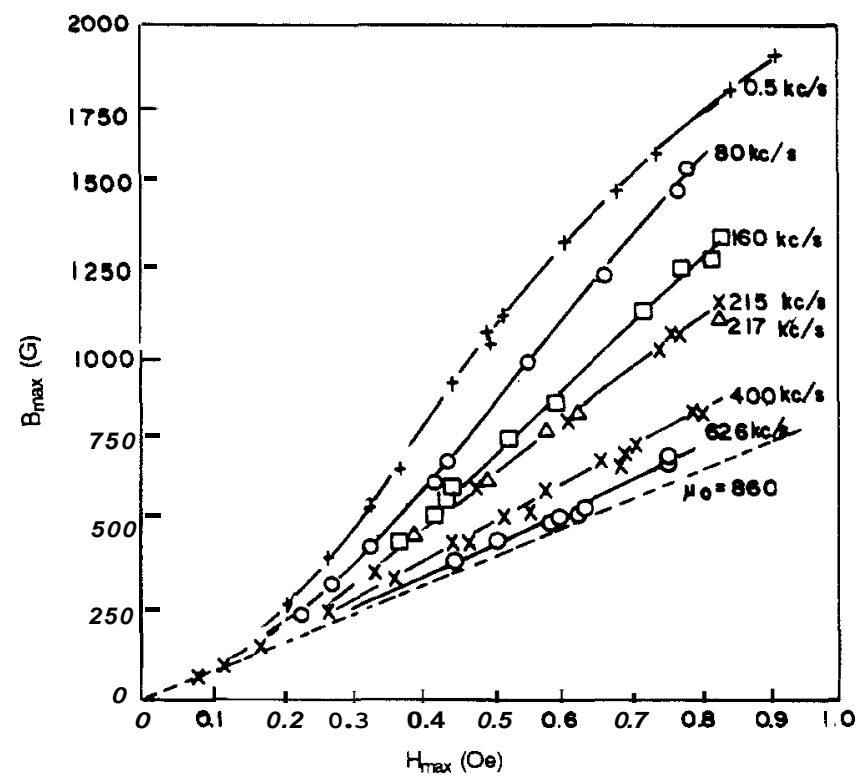

FIG. 7. Magnetization curves of a manganese-ferrous ferrite with a spinel structure $\left(43.5 \mathrm{~mol} \% \mathrm{MnO}\right.$ balance $\mathrm{Fe}_{2} \mathrm{O}_{3}+\mathrm{FeO}$ ) (after Smit and wijn, 1966, Ref. 4). 


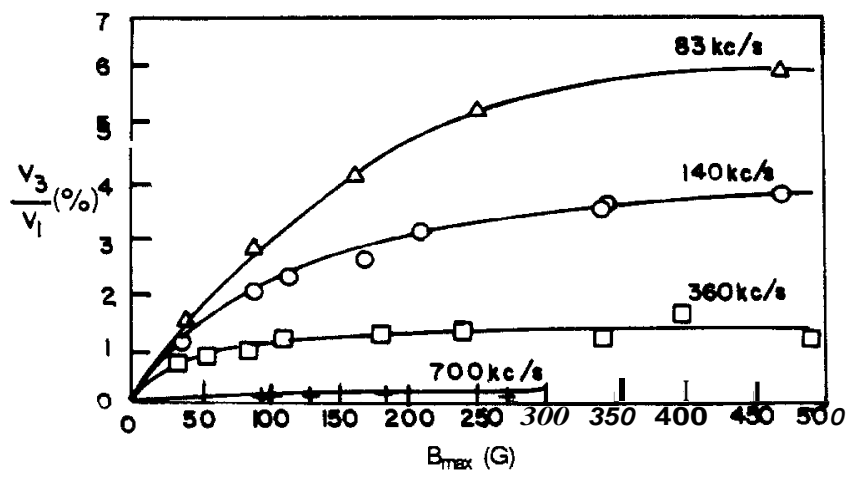

FIG. 8. The distortion $R=V_{3} / V_{1}$ vs the maximum value of the induction $B_{m}$ caused by a sinusoidally varying field strength in a core of the ferrite of Fig. 7 (after Smit and Wijn, 1966, Ref. 4).

current losses in the latter; indeed eddy-current losses are separated from hysteretic losses by plotting the energy dissipation per second as a function of the frequency of the field. Hysteretic losses per cycle are assumed to be frequency independent (hence an extrapolation of the energy dissipation curve to zero frequency gives the hysteretic loss); however, eddy-current losses are proportional to $\omega$ for low frequencies and $\omega^{1 / 2}$ for high frequencies.* One of the important predictions of our theory is that hysteretic losses are not frequency independent; this prediction is clearly of some experimental significance.

So far we have looked at the response of magnets to an oscillating field. We present some experimental data on the response of ferrites to a (rectangular)pulsed magnetic field.

The switching time $\tau$ is defined as the time taken for a half-reversal of the magnetization. Figure 9 shows the switching time as a function of the amplitude of the applied pulse. If the reversal takes place predominantly as a result of irreversible domain-wall displacements, one obtains the following empirical relation: ${ }^{4}$

$$
\left(H_{0}-H_{f}\right) \tau=s,
$$

where $H_{f}$ and $s$ (in msec Oe) are constants for a given ferrite. The threshold strength $H_{f}$ is usually slightly smaller than the coercive field of the ferrite. The rise in the curve of Fig. 9 for values of $H_{0}<H_{f}$ is attributed to the dominant mechanism for magnetization reversal being fast domain-rotation modes rather than irreversible domain. wall motion. ${ }^{4}$

We present our principal results based on studies of the three-dimensional, $\mathbf{O}(N \rightarrow \infty)\left(\boldsymbol{\Phi}^{2}\right)^{2}$ model and the twodimensional Ising model. We study the response of the $\left(\boldsymbol{\Phi}^{2}\right)^{2}$ model to a periodic magnetic field given by $H_{0} \sin (\Omega t)$, where $H_{0}$ and $\Omega$ are the amplitude and frequency of the magnetic field. The Ising model responds to a magnetic field which evolves periodically in a steplike linear fashion (Fig. 26). We shall now summarize our results for the $\left(\boldsymbol{\Phi}^{2}\right)^{2}$ model.

(i) Evolution of the asymptotic $(t \rightarrow \infty)$ shape of the

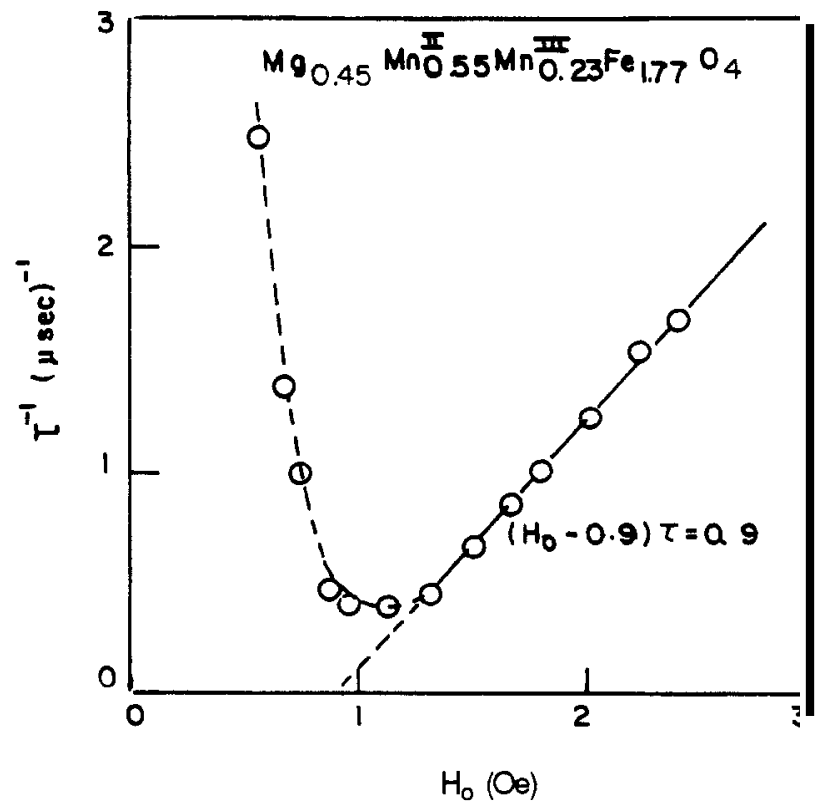

FIG. 9. The reciprocal of the decay time 7 as a function of the amplitude $H_{0}$ (after Smit and Wijn, 1966, Ref. 4).

hysteresis loop for fixed amplitude $H_{0}$ and increasing frequency $\Omega$ [Figs. 10(a)-10(e)]. As a function of $\Omega$ and fixed $H_{0}$, the asymptotic $M-H$ curve changes from the standard spindle-shaped hysteresis loop with saturation (type 1) to a roughly elliptical loop, rounded at the tips, where the major axis is inclined with respect to the $H$ axis (type 2). A subsequent increase in $\mathrm{R}$ rotates the axis of this ellipse until it is parallel to the $H$ axis (type 3 ). As $\Omega$ is increased further, the $M-H$ curve forms an ellipse in the upper half of the $M-H$ plane, which does not close but drifts slowly toward the $H$ axis (type 4). For very large $\Omega$, there is no drift - the loop is an ellipse in the upper half-plane (type 5). Finally as $\Omega \rightarrow \infty$, the area of the ellipse shrinks to zero and the ellipse collapses onto the straight line $\boldsymbol{M}=+M_{\mathrm{eq}}$ (where $M_{\text {eq }}$ is the equilibrium magnetization when $H \rightarrow 0+1$.

(ii) Asymptotic shape of the hysteresis loop in the entire $H_{0}-\Omega$ plane (Fig. 11). When $\Omega$ is held fixed and $H_{0}$ decreased, the shape of the hysteresis loop undergoes the same changes as in (i). We can thus divide the entire $H_{0}-\Omega$ plane into five regions corresponding to the five different types of hysteresis loops shown in Fig. 10. The boundaries between these regions are roughly given by power laws (seeSec. II F).

(iii) Scaling of the area of the hysteresis loop as a function of $H_{0}$, for low $H$, (Fig. 16). Consider only the loops of types 1,2 , and 3. For low values of $H_{0}$ (to be made precise later) and fixed $\mathrm{R}$, the area of the hysteresis loop goes as $\boldsymbol{A} \propto H_{0}^{\alpha}$ with $\boldsymbol{a}=0$. 66. This power law is in qualitative agreement with the Steinmetz law where $a=1.6$. The exponent $a$ appears to be independent of frequency and temperature (we have checked this for two temperatures).

(iv) Scaling of the area as a function of both $H_{0}$ and $\Omega$ 
(Fig. 18). For loops of types 1, 2, and 3 and for low values of $H_{0}$ and $\Omega$, the area of the hysteresis loop exhibits the following simple scaling behavior: $A \propto H_{0}^{\alpha} \Omega^{\beta}$, where $\alpha=0.66 \pm 0.05$ and $\beta=0.33 \pm 0.03$. The exponents $a$ and $\beta$ appear to be independent of temperature.

(v) Scaling of the area of the loop in the limit $\Omega \rightarrow \infty$.
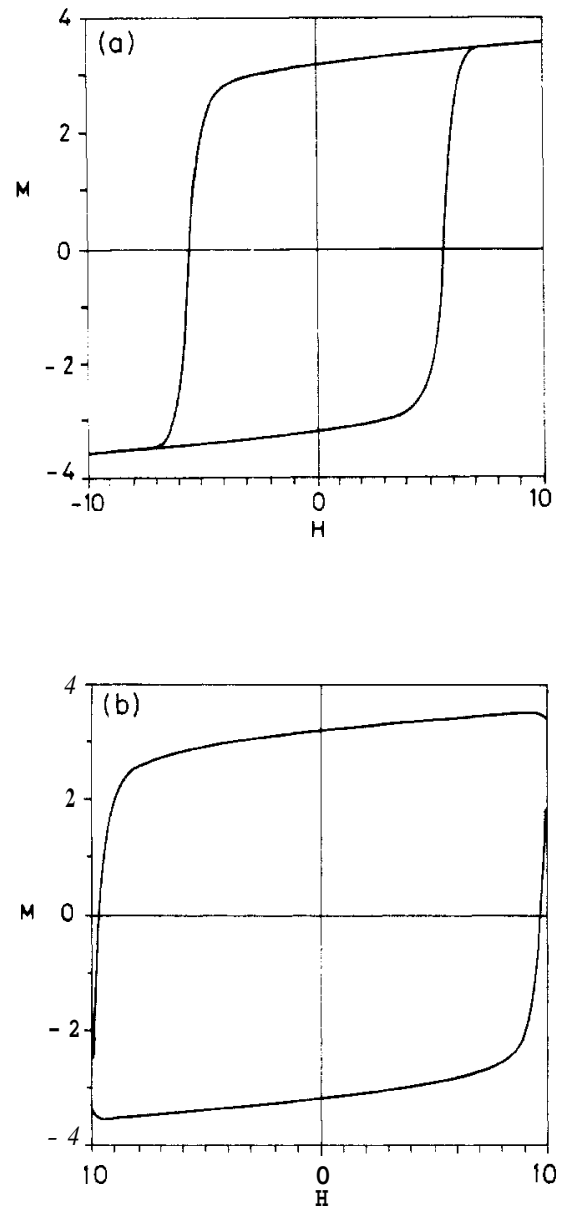

For loops of type 5, a simple analytic treatment yields the elliptical loop of Fig. 10(e) and the area scales as $A \approx H_{0}^{2} \Omega^{-1}$ as $\Omega \rightarrow \infty$ with $H_{0}$ fixed.

(vi) Distortion of the hysteresis loop as a function of $H_{0}$ for various $\Omega$ (Fig. 14). We find, in agreement with experiments, that $\tilde{M}(\omega)$, the Fourier transform of the
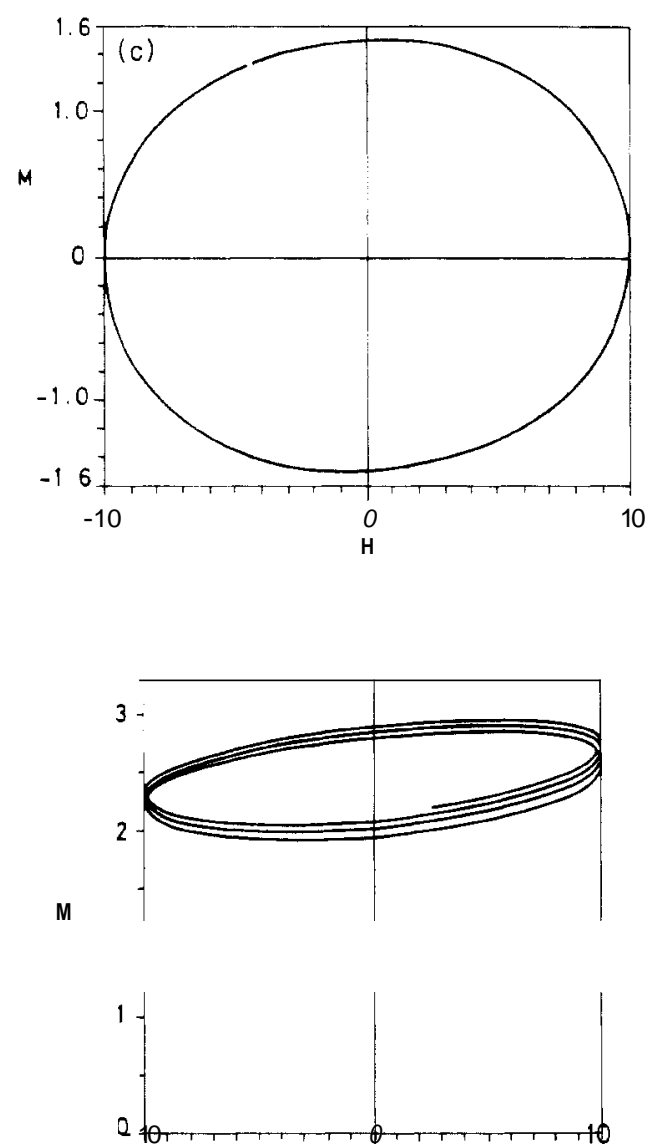

H

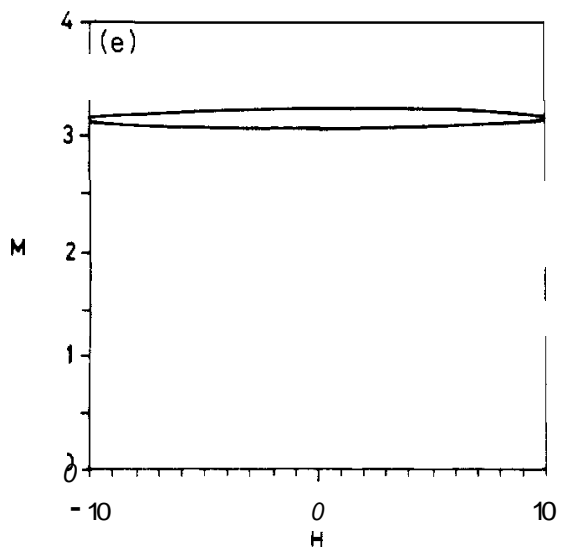

FIG. 10. Typical examples of the five qualitatively distinct hysteresis loops obtained in the large- $N$ approximation. $H_{0}$ is held fixed at 10. $\Omega$ has the following values: (a) $\Omega=0.01$, (b) $\Omega=0.05$, (c) $\Omega=0$. 1, (d) $\Omega=1.2$, and (e) $\Omega=10(r=-10, u=1$ ). 


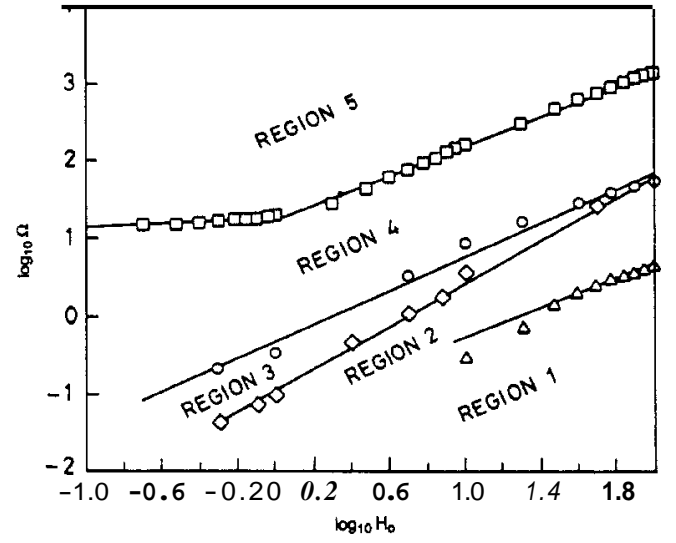

FIG. 11. The stability diagram in the $H$ - $\Omega$ plane showing regions 1-5 in which hysteresis loops of types 1-5 (see Fig. 10), respectively, are obtained. Approximate equations for the boundaries between these regions are given in the text. Lines are drawn to guide the eye through calculated points on these boundaries: squares denote the boundary between regions $\mathbf{4}$ and 5 , circles denote the boundary between regions 3 and $\mathbf{4}$, diamonds denote the boundary between regions 2 and 3, and triangles denote the boundary between regions 1 and 2. $\boldsymbol{r}$ is again held at -10 .

magnetization $M(t)$, has only the fundamental and odd harmonics (Fig. 13) in regions 1,2 , and 3 of the $H_{0} \cdot \Omega$ plane (Fig. 11). The ratio $R$ of the third harmonic $|\tilde{M}(3 \Omega)|$ to the fundamental $|\tilde{M}(\Omega)|$ for fixed $\Omega$ is called the distortion. The distortion increases as a function of $H_{0}$ and then saturates for large $H_{0}$. An increase in $\Omega$ produces a lower distortion. The qualitative behavior seen here is reproduced in experiments (see Fig. 8).

(vii) Mean-field approximation. In a mean-field approximation our model yields standard hysteresis loops only when $H_{0}$ is greater than the spinodal field. The mean-field hysteresis loop (for $\boldsymbol{H}>H_{\mathrm{sp}}$ ) shows a nontrivial frequency dependence; for $H<H_{\text {sp }}$ no hysteresis loop is obtained.

(viii) Response to pulsed magnetic fields. The response of the magnetization to a pulsed magnetic field shows the existence of three time scales associated with three distinct dynamical processes. These time scales have a power-law dependence on the magnitude of the pulsed field for small fields. We show that these time scales are related to the boundaries separating the regions of qualitatively distinct hysteretic behavior.

Our data for the Monte Carlo simulation of the hysteretic response of the two-dimensional Ising model are not as extensive as those of the N-component $\left(\boldsymbol{\Phi}^{2}\right)^{2}$ model. With our computing facilities, a detailed quantitative study of hysteresis in Ising systems is not feasible. Nevertheless we obtain the following qualitative results.

(i) Variation with the frequency of the field. Keeping the amplitude of the field, the temperature, and the size of the lattice fixed, an increase in the frequency of the field changes the shape of the hysteresis loop from types $1 \rightarrow 2 \rightarrow 3 \rightarrow 4 \rightarrow 5$ (the classification of the loops is the same as above). We find in loops of type 1 that the coercive field (and hence the area) increases with increasing frequency.

(ii) Variation with the amplitude of the field. Keeping the frequency of the magnetic field, the temperature, and the size of the lattice fixed, an increase in the amplitude of the field changes the shape of the hysteresis loops from types $5 \rightarrow 4 \rightarrow 3 \rightarrow 2 \rightarrow 1$. The type 1 loops are more squarish than those obtained in the $\left(\boldsymbol{\Phi}^{2}\right)^{2}$ model. The coercive fields are of the order of the exchange coupling $J$ for the frequency and amplitude ranges that we consider $\left(0.2 J \leq H_{0} \leq 2 J\right)$.

(iii) Variation with temperature. In loops of type 1, the coercive field (and hence the area) decreases with increasing temperature. For $T>T_{c}$, the hysteresis loop disappears - the magnetization simply follows the field. Keeping the amplitude and frequency of the field and the lattice size fixed, a decrease in temperature changes the loops from types $1 \rightarrow 2 \rightarrow 3 \rightarrow 4 \rightarrow 5$.

(iv) Variation with lattice size. In loops of type 1 , an increase in the lattice size decreases the coercivity (this size dependence of the coercivity is very weak). The remnant magnetization increases with increasing lattice size. Keeping the temperature, the amplitude, and the frequency of the field fixed, an increase in the lattice size changes the loops from types $5 \rightarrow 4 \rightarrow 3 \rightarrow 2 \rightarrow 1$.

The remaining part of this paper is organized as follows: In Sec. II we present the calculations for the $\mathrm{O}(N)$ symmetric $\left(\boldsymbol{\Phi}^{2}\right)^{2}$ model. In Sec. III we describe our Monte Carlo simulations of the Ising model in two dimensions. In Sec. IV we compare our study with earlier, phenomenological theories of hysteresis. In Sec. V we examine the experimental relevance of our study.

\section{HYSTERESIS IN THE O $(N)$-SYMMETRIC $\left(\Phi^{2}\right)^{2}$ THEORY}

We begin our study of hysteresis by investigating the response of an $\mathrm{N}$-component $\left(\boldsymbol{\Phi}^{2}\right)^{2}$ model with $O(N)$ symmetry in three dimensions to an external, periodic magnetic field. The order parameter, namely the magnetization, is not conserved and its evolution is described by model- $\boldsymbol{A}$ (nonconserved order parameter) dynamics. Our analysis is valid to first order in a $1 / N$ expansion - it is thus exact in the $N=\infty$ limit.

In Sec. II A we describe our model. In Sec. II $\mathbf{B}$ we use it to study hysteresis loops in the $N=\infty$ limit. Section IIE contains a similar study using a mean-field theory. Section II F is a study of the response of this model to a pulsed magnetic field.

\section{A. Description of the model}

The order parameter $\Phi$ obeys the following Langevin equation:

$$
\frac{\partial \Phi_{\alpha}}{\partial t}=-\Gamma \frac{\delta(\beta F)}{\delta \Phi_{\alpha}}+\eta_{\alpha},
$$

where $\eta_{\alpha}$ is a Gaussian white noise:

$$
\left\langle\eta_{\alpha}(\mathrm{x}, t)\right\rangle=0
$$




$$
\left\langle\eta_{\alpha}(\mathbf{x}, t) \eta_{\beta}\left(\mathbf{x}^{\prime}, t^{\prime}\right)\right\rangle=2 \Gamma_{1} \delta_{\alpha \beta} \delta\left(\mathbf{x}-\mathbf{x}^{\prime}\right) \delta\left(t-t^{\prime}\right),
$$

and $\beta F$ is the free-energy functional

$$
\begin{aligned}
\beta F=\int d^{3} x \mid & \frac{1}{2} J\left(\nabla \Phi_{\alpha}\right)\left(\nabla \Phi_{\alpha}\right)+\frac{r}{2}\left(\Phi_{\alpha} \Phi_{\alpha}\right) \\
& \left.+\frac{u}{4 N}\left(\Phi_{\alpha} \Phi_{\alpha}\right)^{2}-\sqrt{N} H_{\alpha} \Phi_{\alpha}\right) .
\end{aligned}
$$

Since $\boldsymbol{\Phi}$ is an N-component vector, $\boldsymbol{\Phi}_{\alpha} \boldsymbol{\Phi}_{\alpha}$ (sum over repeated indices) scales as $\mathrm{N}$. The $N$ dependence of the terms linear and quartic in $\Phi_{\alpha}$ thus ensures that the free energy scales as $\mathrm{N}$. The coefficient $r=T-T_{c}^{\mathrm{MF}}$, where $T$ is the temperature and $T_{c}^{M F}$ is the mean-field critical temperature. In all our subsequent analysis we take $J$ to be equal to 1. The magnetic field, constant and uniform, points along the $\alpha=1$ direction in spin space, i.e., $H_{\alpha}=H \delta_{\alpha, 1}$.

A sufficient condition for the attainment of equilibrium is $\Gamma=\Gamma_{1}$. The stochastic differential equation (3) is equivalent to an infinite hierarchy of differential equations for the cumulants of $\Phi_{\alpha^{\prime}}$ In the $N=\infty$ limit, this infinite hierarchy of differential equations is truncated leading to the following coupled integrodifferential equation $\sim$ !e

$$
\begin{aligned}
& \frac{d M(t)}{d t}=\frac{1}{2}\left[M(t) A(t)+H_{0} \sin (\Omega t)\right], \\
& A(t)=-\left[r+u M^{2}(t)+u S(t)\right], \\
& S(t)=\frac{1}{2 \pi^{2}} \int_{0}^{1} q^{2} C_{1}(q, t) d q,
\end{aligned}
$$

and

$$
\frac{d C_{\perp}}{d t}(q, t)=-\left[q^{2}-A(t)\right] C_{\perp}(q, t)+1 .
$$

$\boldsymbol{M}(t)$ is the magnetization and is given by $\left\langle\Phi_{1}(\mathbf{q}, t)\right\rangle$ while $C_{1}(q, t)$ is the transverse correlation function $\left\langle\Phi_{\alpha}(\mathbf{q}, t) \Phi_{\alpha}(-\mathbf{q}, t)\right\rangle$ with $\alpha \neq 1$. Higher-order (order $>2$ ) cumulants of the order-parameter distribution go to zero as $N \rightarrow \infty$. The longitudinal correlation function $\left\langle\Phi_{1}(\mathbf{q}, t) \Phi_{1}(-\mathrm{q}, t)\right\rangle$ is dominated by the transverse correlation function by a factor of $1 / N$. It can be easily shown (see the Appendix) that if the initial magnetization is homogeneous then the dynamical equations (7a)-(7d) maintain the homogeneity of the magnetization. Since we are interested in the magnetic response of the spin system in its ferromagnetic phase, the initial conditions required to solve the above dynamical equations are the values of the magnetization and the transverse correlation function in equilibrium at a temperature $r<r_{c}=-u / 2 \pi^{2}$ and magnetic field $H_{\alpha} \rightarrow(0+) \delta_{\alpha, 1}$. The equilibrium magnetization is

$$
M \equiv\left\langle\Phi_{1}(q)\right\rangle=\left[\left(r_{c}-r\right) / u\right]^{1 / 2}
$$

while $\left\langle\Phi_{\alpha}(\mathbf{q})\right\rangle=0$, for a\# 1. The transverse correlation function

$$
C_{1}(q) \equiv\left\langle\Phi_{\alpha}(q) \Phi_{\alpha}(-\mathbf{q})\right\rangle=1 / q^{2} \text { for } \alpha \neq 1 .
$$

The transverse correlation function diverges at $q=0$ because of low-energy spin-wave excitations. The longitudinal correlation function"

$$
C_{\|}(q) \equiv\left\langle\Phi_{1}(\mathbf{q}) \Phi_{1}(-\mathbf{q})\right\rangle=1 / q \tan ^{-}(q / 2 b),
$$

where $b^{2}=H_{a} / 2 M$, also diverges at $q=0$.

These are the only nonzero cumulants in the $N=\infty$ theory. The off-diagonal components of the two-point correlation function

$$
C_{\alpha \beta}(q) \equiv\left\langle\Phi_{\alpha}(q) \Phi_{\beta}(-q)\right\rangle
$$

are zero by rotational invariance about the $\alpha=1$ axis. Higher-order cumulants are smaller by factors of $N^{-1}$ and therefore can be ignored when $N=\infty$.

The equilibrium quantities displayed here will be used as initial data for the dynamical equations (7).

Equations 7(a) - 7(d) tell us that the magnetic field $H(t)$ drives the $\alpha=1$ component of $\left\langle\Phi_{\alpha}(\mathbf{q}, t)\right\rangle$ (the magnetization). $\left\langle\Phi_{1}(\mathbf{q}, t)\right\rangle$ is coupled to other components of $\left\langle\Phi_{\alpha}(\mathbf{q}, t)\right\rangle$ through the transverse correlation function, i.e., it dissipates into the transverse modes. When $M(t)=0, C_{1}$ is a maximum and vice versa. When $H_{0}=0$, the asymptotic solutions of the above equations reduce to Eqs. (8) and (9). As $t \rightarrow \infty$ (stationary solutions), the magnetization and correlation function attain their equilibrium values. In the above equations we have redefined time to incorporate the $\Gamma$ term. We measure time in units of $(2 \Gamma)^{-1}$ (the spin-lattice relaxation time, typically of the order of $10^{-8} \mathrm{sec}$ ).

\section{B. Results for the $N=\infty$ limit}

Equations (7) are a set of nonlinear integrodifferential equations. These equations cannot be solved analytically for all $H_{0}$ and $\Omega$. Therefore, we solve these equations numerically - we use 20- or 24-point Gauss quadrature routines for evaluating the integrals, and a finitedifference Euler and an adaptive-size Runge-Kutta scheme to solve the differential equations. At high frequencies, the time step for the differential equation should be small. Such small time steps make the CPU time required for solving the differential equations up to a time $t$ very large. We thus use the Gear method, ${ }^{12}$ especially suited for tackling such stiff differential equations, for solving the differential equations (7) at high frequencies $\boldsymbol{\Omega}$. These equations are solved to obtain $\boldsymbol{M}$ as a function of $t$. We can thus plot $M(t)$ versus $H(t)$ for various $r, u, H_{0}$, and $\Omega$ and study the evolution of the shapes of hysteresis loops as these parameters are varied.

The numerical solution of Eqs. (7) gives the time evolution of the magnetization for various values of the parameters $\Omega, H_{0}, \boldsymbol{r}$, and $\boldsymbol{u}$. The magnetization settles onto a periodic orbit only after a time $\tau_{t}$ larger than the time taken for all the transients to die down; $\tau_{t}$ depends on the frequency and amplitude of the magnetic field-it increases as $\Omega$ increases and decreases as $H_{0}$ increases. This is true for all $\Omega$ and $H_{0}$ except those that lie in region 5 of Fig. 11. All our results are for this asymptotic loop. 


\section{Variation of the shape of the loop with frequency}

In the Introduction we discussed the behavior of the hysteresis loop when $\Omega=0$ and $\infty$. Here we show how the shape of the hysteresis loop evolves from its $\Omega=0$ to its $\Omega=\infty$ shape for our $\left(\boldsymbol{\Phi}^{2}\right)^{2}$ model.

For fixed $H_{0}$ and varying $\Omega$, the hysteresis loops have five qualitatively different asymptotic shapes [Figs. 10(a)-10(e)], which interpolate naturally between the $\Omega \rightarrow 0$ and $\Omega \rightarrow \infty$ behaviors discussed above. If we fix $H_{0}$, then, at low il, we obtain the commonly observed squarish loop of Fig. 10(a) (type 1), which shows $\boldsymbol{M}$ saturating at high fields. ${ }^{13}$ As il increases, this loop does not show a saturation of $\boldsymbol{M}$ and is rounded at its corners [Fig. 10(b), type 2]. A further increase in il makes this loop turn until the semimajor axis is $M=O$ [Fig. 10(c), type 3]. At even higher values of $\Omega$, the loop lies in the upper part of the $M-H$ plane since we use the initial condition $M(t=0)=+M_{\text {eq }}$; this loop does not close but drifts downwards very slowly [Fig. 10(d), type 4]. As far as we can tell, the loops do not converge geometrically onto an asymptotic loop that is closed. At very large values of $\Omega$, this drift ceases to be visible on the scale of Fig. 10(e) (type 5); the loop becomes more and more elliptical and narrower as $\Omega$ increases and, as $\Omega \rightarrow \infty$, the loop collapses onto the straight line $M(t)=+M_{\text {eq }}$ as expected.

\section{Stability diagrum}

We now keep the frequency $\Omega$ constant and analyze the change in the shapes of the hysteresis loops as a function of $H$,. We start with a large enough value of $H_{0}$ to saturate the magnetization. The hysteresis loop obtained is of type 1. For a lower value of $H_{0}$ the loop does not display saturation - the corresponding hysteresis loop is of type 2. A further decrease in $H_{0}$ rotates the major axis of the roughly elliptical loop towards the $H$ axis (type 3 ). As $H_{0}$ decreases, the loop changes to a type- 4 and then to a type-5 hysteresis loop.

We therefore see that the frequency ranges in which these five shapes obtain depend on $H_{0}$ : in the $H_{0}-\Omega$ plane we plot a stability diagram (Fig. 11) which shows the regions 1-5 where these five shapes are obtained asymptotically.

The boundaries separating the different regions of Fig. 11 should not be thought of as sharp boundaries; the changes in the shapes of the loops occur gradually. We have chosen the following criteria to determine the boundaries between the five regions given above: (a) Regions 1 and 2. As we traverse the loop in the first quadrant of the $M-H$ plane, $d^{2} M / d H^{2}$ changes sign (does not change sign) if the point $\left(\Omega, H_{0}\right)$ lies in region 1 (region 2 ) of Fig. 11. (b) Regions 2 and 3. In region 2 (region 3), $M$ does not change sign (changes sign) as $H(t)$ passes through its maximum value $H_{0}$. (c) Regions 3 and 4. In region 3 (region 4), the lower value of $M$ at $H=0$ is negative (positive), after the field $H$ has gone through 100 cycles. (d) Regions 4 and 5. In region 4 (region 5), the ratio

$$
[M(t=0)-M(t=T)] / M(t=0)
$$

has a value greater than (less than) 0.01 , where the ratio is evaluated at $H=0$ and $T$ is the time'required for 100 cycles of the field $H$. The boundaries between the regions obey approximate power laws with exponents that depend on the range of $H_{0}$. The boundary between regions 1 and 2 is given by $\Omega \approx H_{0}^{0.95}$ for $10 \leq H_{0} \square 00$. The boundary between regions 2 and 3 is $\Omega \approx H_{0}^{1.35}$ for $10 \leq H_{0} \mathbf{I} 100$ and $\Omega \approx H_{0}^{1.5}$ for $0.1 \leq H_{0} \leq 10$. Regions 3 and 4 are divided by the boundary whose equation is $\Omega \approx H_{0}^{1.08}$ for $0.1 \leq H_{0} \square 00$. The region 4-region 5 boundary is given by $\Omega \approx H_{0}^{0.97}$ for $1 \leq H_{0} \leq 100$ and $\Omega \approx H_{0}^{0.13}$ for $0.1 \leq H_{0}<1$.

To compare our results with those obtained experimentally for real magnets, we must specify the scales of $H_{0}$ and $t$. The scale of $H_{0}$ can be set by the molecular field, which is typically $10^{7}$ Oe. The scale of $\mathrm{t}$ is set by $(2 \Gamma)^{-1}$, which is related to a microscopic relaxation time such as the spin-lattice relaxation time. As we discuss in Sec. V, the simplest estimate for $2 \Gamma$ can be obtained from the width of a typical ferromagnetic-resonance line; this yields $2 \Gamma \approx 10^{8} \mathrm{~Hz}$. Thus, the loops of region 3 of Fig. 11 should be obtained at easily accessible frequencies only if $H_{0}$ is very small (an extrapolation of the stability boundaries of Fig. 11 yields, for $\Omega=100 \mathrm{~Hz}$, the following bounds for region 3: $10^{1.8} \leq H_{0}<10^{3.65} \mathrm{Oe}$ ). We are not aware of any experimental stability diagram such as the one we portray in Fig. 11.

\section{Time evolution of the mugnetizution}

Since the asymptotic hysteresis loops obtained are closed (except those of type 4), the magnetization must be a periodic function of time, with the same period as the magnetic field $2 \pi / \Omega$. To obtain a hysteresis loop, the magnetization should be phase shifted with respect to the magnetic field. Figures 12(a)-12(e) show the time evolution of the magnetization and the magnetic field in each of the five regions of Fig. 11. Note the shift in phase of the magnetization with respect to the magnetic field. The evolution of the magnetization shown in Figs. 12(a)-12(c) has two parts - one is the slow variation of the magnetization near the extrema and the other is the fast jump of the magnetization in the region of the curve where the magnetization changes sign. As we will argue in Sec. II E, these slow and fast variations of the magnetization can be understood roughly by using the mechanical analogue of the $\Phi^{4}$ theory: The slow variation is an indication of the time taken for the magnetization to decay from the metastable minimum. The fast variation is a reflection of the fast relaxation towards the stable minimum. The amplitude of the magnetization curve increases with $H_{0}$ while the phase difference between the magnetization and the magnetic field increases with $\Omega$ (for values of $\Omega$ in regions 1-3). The magnetization curve of Fig. 12(d) (belonging to type-4 hysteresis) is not periodic. The difference between the successive maxima, namely $M(t,)-,M\left(t_{n-1}\right)$ [where $M\left(t_{n}\right)$ is the magnetization at the $n$th maximum] decreases arithmetically. The oscillations do not cross the $M=0$ axis. In Fig. 12(e), $\boldsymbol{M}(t)$ is periodic. It resembles a sinusoidal curve which is 
symmetric not about the origin but about $M=M_{\text {in }}$ (initial magnetization).

In Figs. 13(a)-13(e) we show Fourier transforms $\tilde{M}(\omega)$ of $M(t)$ shown in Figs. 12(a)-12(e), respectively. Figures $12(a)-12$ (c) resemble periodic square waves. The Fourier transform of a square wave of frequency $\Omega$ is nonzero only for the fundamental frequency $\Omega$ and its odd harmonics. We see that the Fourier transform of $\boldsymbol{M}(t)$ in regions $1-3$ is also appreciable only for the fundamental $\Omega$ and its odd harmonics. The amplitudes of the higher Fourier components $(3 \Omega, 5 \Omega$, etc.) decrease exponentially. The appearance of only odd harmonics can be under- stood from Eq. (13) which is the integral equation corresponding to the differential equation (7). This equation contains a driving term $\delta(\omega-\Omega)$ and a convolution of three Fourier components of the magnetization. An iterative solution of Eq. (13) reveals that only $\tilde{\boldsymbol{M}}(\Omega)$ and its odd Fourier components pick up nonzero values. The Fourier transform of Fig. 12(d) has all Fourier components (including the even ones) and a large $\tilde{M}(\omega=0)$ component [Fig. 13(d)]. The reason for the appearance of all Fourier components is that $M(t)$ is not periodic. $\tilde{M}(\omega=0) \neq 0$ since the magnetization is not symmetric about the $H$ axis. Figure 13(e) is the Fourier transform of
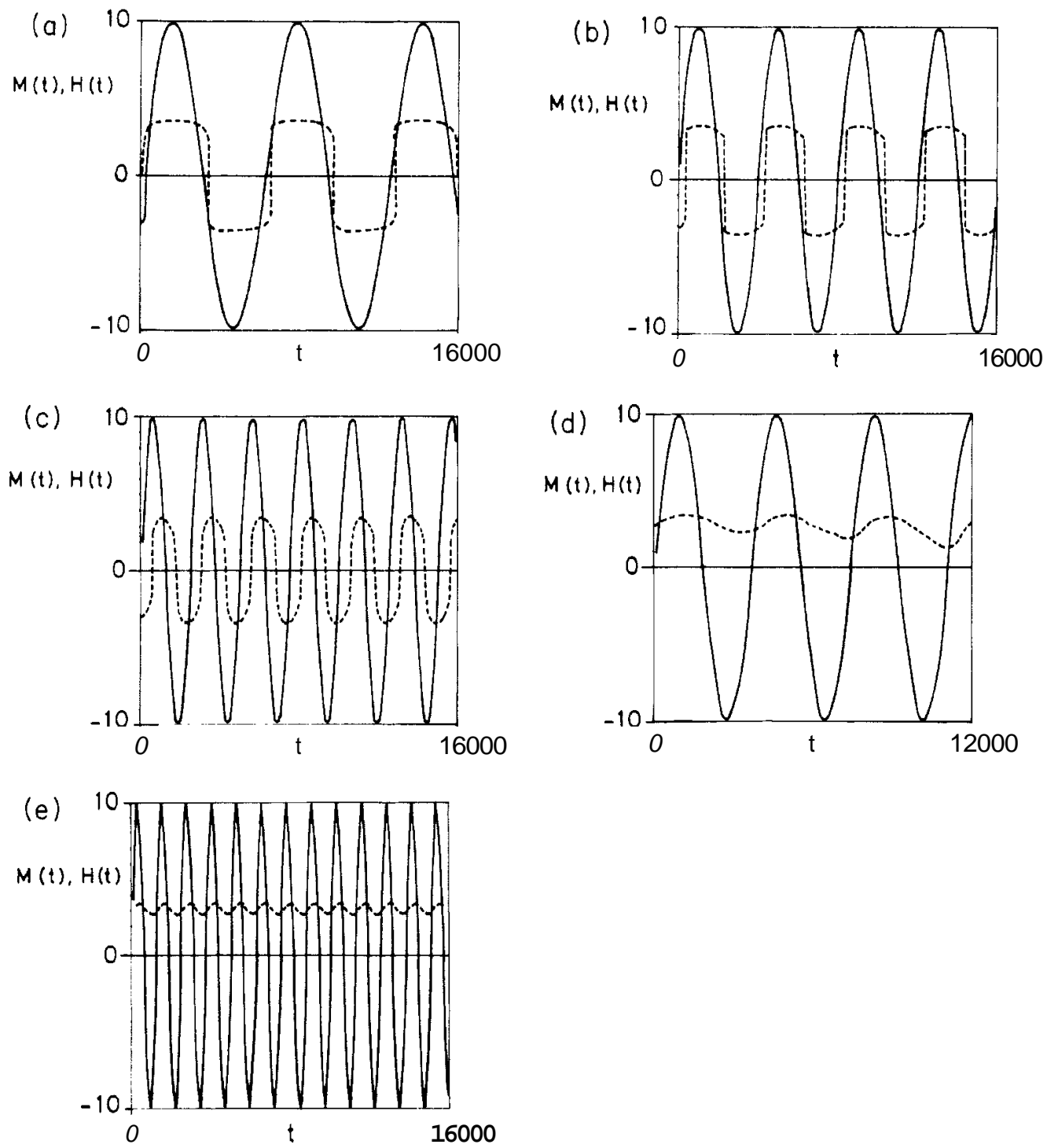

FIG. 12. Time evolution of the magnetization (dotted line) and the magnetic field (solid line) in the five regions of Fig. 11. The frequency is varied from region $1(\mathrm{a})$ to region $5(\mathrm{e})$ keeping the amplitude fixed $\left(H_{0}=10, r=-10\right.$, and $\left.u=1\right)$. 
the loop of type 5 . It possesses large $\tilde{M}(\omega=0)$ and $\tilde{M}(\omega=\Omega)$ Fourier amplitudes. The higher harmonics go to zero as $\Omega$ increases. This indicates that the hysteresis loop is an ellipse about $M=M_{\text {in }}[=\tilde{M}(\omega=0)]$.

We define the distortion $R$ as

$$
R \equiv|\tilde{M}(3 \Omega)| /|\tilde{M}(\Omega)|
$$

(see the Introduction). This quantity is a measure of the distortion of the hysteresis loop from ellipticity or the de. viation of the extrema of $M(t)$ from those of a pure sine curve. $R$ is therefore small in region 5 and increases monotonically as we go from region 5 to region 1 of Fig. 11. This increase shows clearly in Fig. 14 where, at fixed $\Omega$, we go from region 5 to region 1 by increasing $\boldsymbol{H}$, The saturation of $R$ at large values of $H$, is in qualitative agreement with experiments on real magnets (see Fig. 8). Keeping $H_{0}$ fixed, an increase in $\Omega$ makes the hysteresis loop more elliptical. Therefore, for fixed $H, \boldsymbol{R}$ decreases as $\Omega$ increases [compare Figs. 14(a) and 14(b)].

$|\bar{M}(\omega)|$

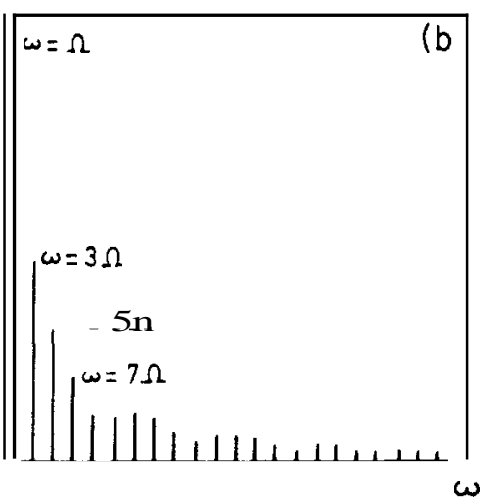

$|\tilde{M}(\omega)|$

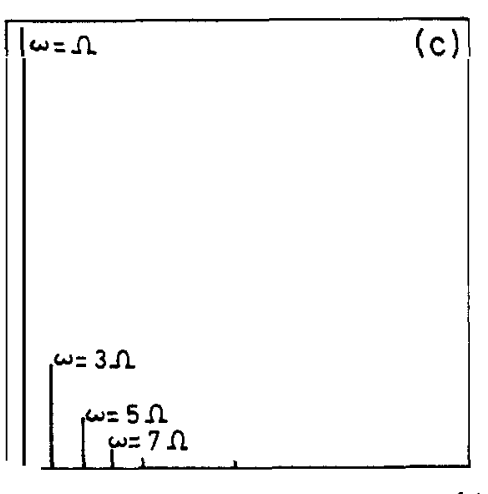

$|\tilde{M}(\omega)|$

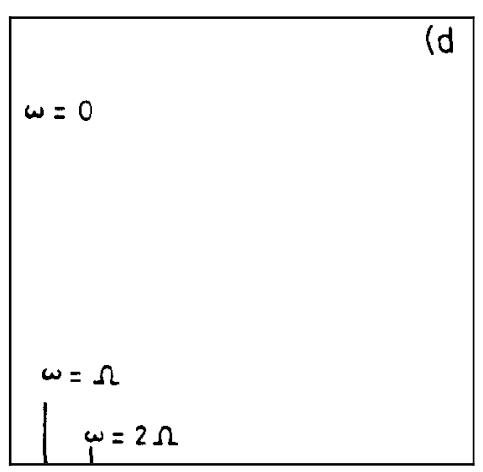

$\omega$

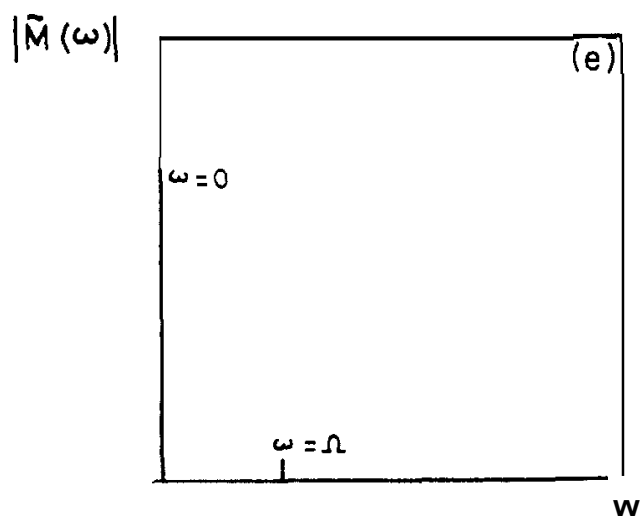

FIG. 13. Fourier transforms of the time evolution of the magnetization shown in Figs. 12(a)-12(e). 


\section{Variation of transverse correlation function}

The dynamical equations (7) reveal that the magnetization is driven by the external magnetic field and constantly dissipates by coupling to the transverse fluctuations of the order parameter. Thus, a decrease in the magnetization is accompanied by an increase in the transverse correlation function. From Eq. (7b) we see that the maximum value of $C_{1}$ at all times is at $q=0$.

From Eq. (7) we would expect $C_{\perp}$ to attain its maximum value (for a fixed $q$ ) whenever $M$ goes through zero. Moreover, $C_{\perp}$ should be independent of the sign of $M(t)$. From these arguments, $C_{1}$ should be periodic with frequency $2 \mathrm{R}\left(C_{1}\right.$ is, of course, always positive). This is seen in Fig. 15 for regions 1-3. It is also seen that, for smaller $q, C$, has a larger amplitude. When the frequency of the external magnetic field is large (region 5), the variation in the transverse correlation function takes place over a much slower time scale compared to the high-frequency oscillations of the magnetic field. Thus,
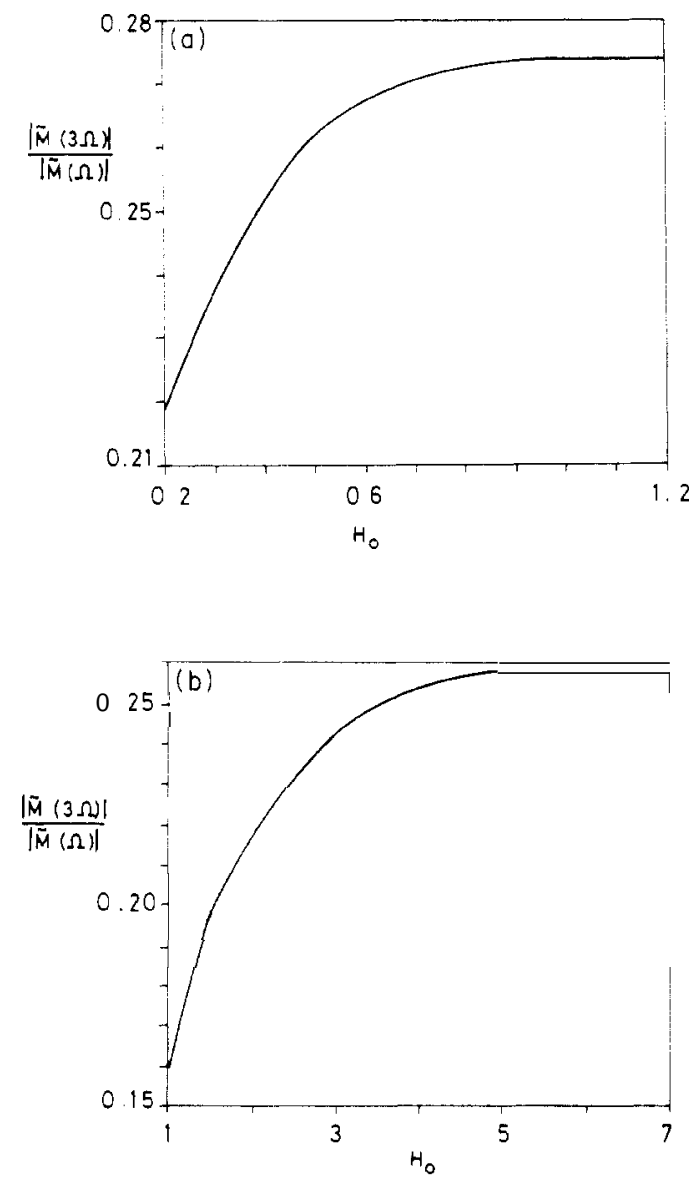

FIG. 14. The ratio $R \equiv|\overline{\boldsymbol{M}}(3 \Omega)| /|\overline{\boldsymbol{M}}(\Omega)|$, called the distortion, as a function of $H_{01}$ for two values of R: (a) $\Omega=0.001$ and (b) $\Omega=0.1$.
$C_{1}$ does not deviate very much from its equilibrium value of $1 / q^{2}$ for large $\Omega$. As $\Omega \rightarrow \infty, C_{\perp}(q, t)=c_{\perp}^{\text {in }}(q)$ [the initial value of $\left.C_{1}(q)\right], S(t)$, which is $\int C_{1}(q, t) d^{3} q$, is a measure of the "volume" under the $C_{1}$ surface. $S(t)$ is also periodic with half the period of $M(t)$ and reaches a maximum whenever $M(t)=0$ (in regions $1-3$ ).

\section{Variation of the area of the loop}

Given a hysteresis loop, we can easily determine its area. The area of the hysteresis loop is a measure of the energy dissipated in going through a whole cycle of the magnetic field. Let us fix the temperature $r$ and the frequency $\Omega$. The areas of loops of types $1-3$ should increase as a function of $H_{0}$. We shall restrict ourselves to small $H$, i.e., $H_{0} \ll H_{\text {sp }}$, where $H_{\text {sp }}$ is the mean-field spinodal magnetic field. We find that the area of the asymptotic hysteresis loops scales with $H_{0}$ as

$$
A \propto H_{0}^{\alpha}
$$

where $\alpha=0.66 \pm 0.05$. This dependence is true for a wide range of values of $\Omega$ (over three decades of frequency, Fig. 16). It also seems to be independent of temperature (we have checked it for $r=-10$ and -2 ). This powerlaw dependence on $H_{0}$ is in qualitative though not quantitative agreement with the Steinmetz law (see the Introduction) where $\alpha=1.6$ for a wide variety of soft magnets.

Let us now fix $H_{0}$ and $\boldsymbol{r}$ and compute the area of the loop as a function of the frequency R. As we have seen, an increase in $\Omega$ changes the hysteresis loops from those of type 1 to those of types $\mathbf{2 - 4}$ and eventually 5 . The area of the hysteresis loop for $\Omega \rightarrow \infty$ and $\Omega \rightarrow 0$ is zero. As $\Omega$ increases from zero the area of the hysteresis loop increases. The area thus goes through a maximum and then decreases as a function of $H_{0}$ (Fig. 17).

We now concentrate our attention on low $H_{0}\left(\ll H_{\mathrm{sp}}\right)$ and low $\Omega$ (we are thus in regions 1,2 , or 3 ). The dependence of the area on $H_{0}$ and $\Omega$ is interesting: We find that the area has a power-law dependence on the amplitude of the field and the frequency; the best fit to numerical data is given by the following scaling form:

$$
A \propto H_{0}^{\alpha} \Omega^{\beta}
$$

where $\alpha=0.66 \pm 0.05$ and $\beta=0.33 \pm 0.03$. (The square of the correlation coefficient for this fit is equal to 0.9967.) Figure 18(a) exhibits this scaling form plotted for a wide range of frequencies: $10^{-4} \leq \Omega \leq 10^{-1}$. The plot is for a temperature $r=-10$. The same power-law dependence is found for a higher temperature $r=-2$ [Fig. 18(b)]. We thus conclude that the exponents $\alpha$ and $\beta$ are temperature independent. The temperature dependence of the area $\boldsymbol{A}$ is contained in the amplitude

$$
A \approx \chi(r) H_{0}^{\alpha} \Omega^{\beta}
$$

$\chi(\boldsymbol{r})$ is a monotonically decreasing function of $\boldsymbol{r}$. This scaling form is valid for low $H_{0}$ and $\Omega$, i.e., in the regions $1-3$ of Fig. 11. 

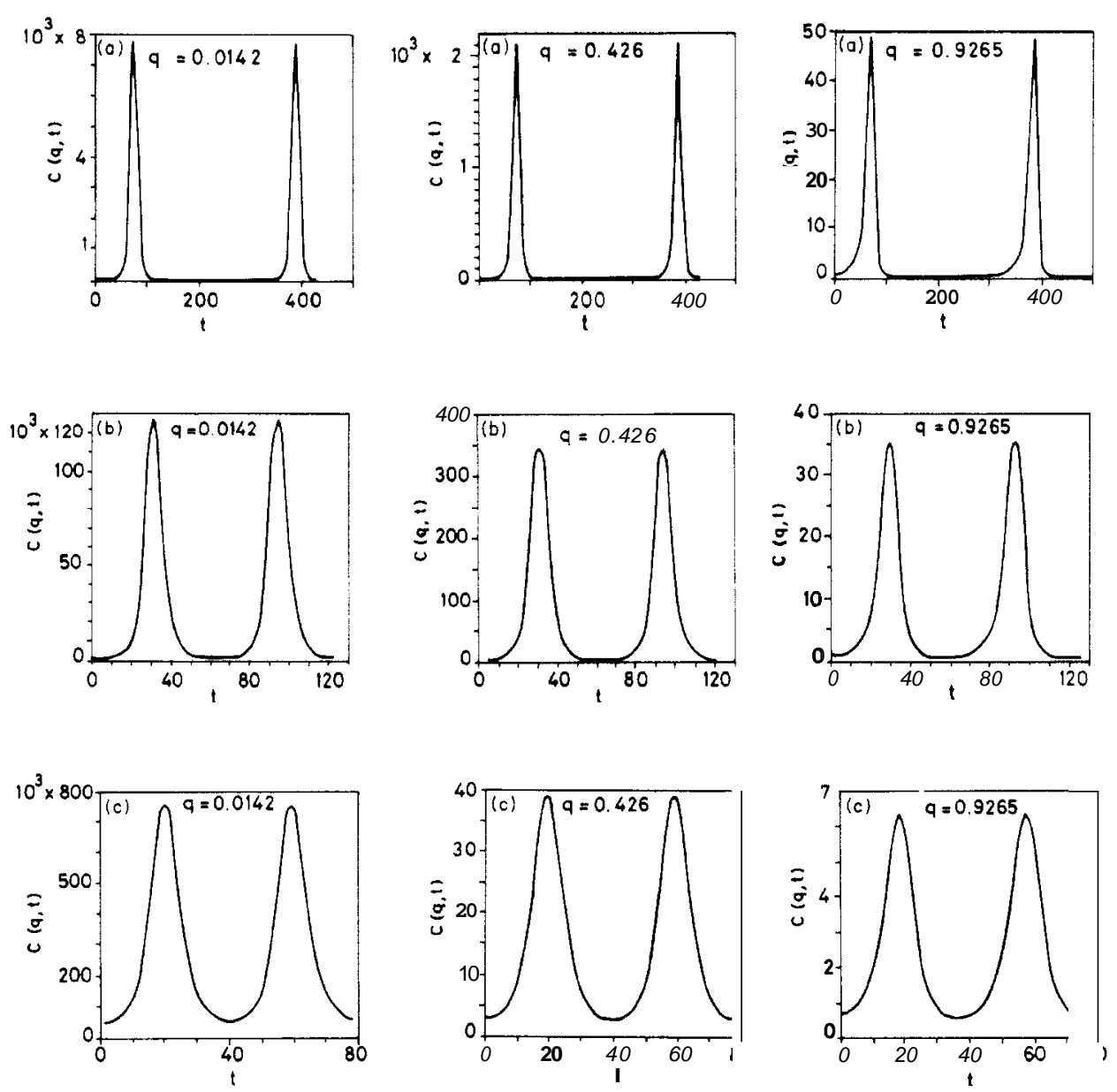

FIG. 15. The transverse correlation function vs time for three different values of the wave vector $q$ in (a) region 1 , (b) region 2 , and (c) region 3 of Fig. $11\left(H_{0}=10, \mathrm{r}=-10\right.$, and $\left.u=1\right)$.
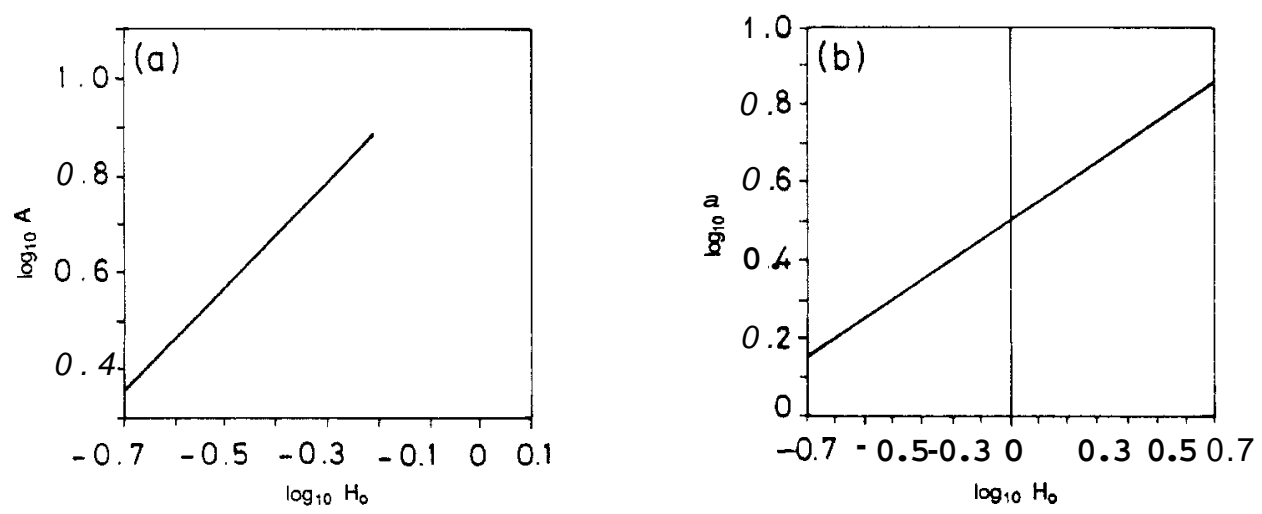

FIG. 16. Scaling of the area of the hysteresis loop with $H_{0}$, keeping $\Omega$ constant: (a) $\Omega=0.01$, (b) $\Omega=0.001$. The area scales as $H_{0}^{0.66}(r=-10$ and $u=1)$. 


\section{High-frequency results}

Equations (7) can be solved analytically in the highfrequency limit, i.e., for type-5 hysteresis loops. This can be seen by Fourier transforming Eqs. (7) with respect to $t$. This converts the differential equation into a nonlinear in- tegral equation. Define

$$
\tilde{M}(\omega)=\frac{1}{\sqrt{2} \pi} \int_{0}^{\infty} M(t) e^{i \omega t} d t
$$

and similarly $\widetilde{\boldsymbol{C}}_{1}(q, \omega)$. Then Eqs. (7) give

$$
\begin{aligned}
-i \omega \tilde{M}(\omega)= & -r \tilde{M}(\omega)+\left(H_{0} / 4 i\right)[\delta(\omega+\Omega)-\delta(\omega-\Omega)]-\frac{u}{2\left(2 \pi^{3 / 2}\right)} \int_{0}^{\infty} \int_{0}^{\infty} \tilde{M}\left(\omega_{1}\right) \tilde{M}\left(\omega_{2}-\omega_{1}\right) \tilde{M}\left(\omega-\omega_{2}\right) d \omega_{1} d \omega_{2} \\
& -\frac{u}{2\left(2 \pi^{3 / 2}\right)} \int_{0}^{\infty} \frac{1}{4 \pi^{2}} \int_{0}^{1} \tilde{C}_{1}\left(q, \omega_{1}\right) q^{2} d q \tilde{M}\left(\omega-\omega_{1}\right) d \omega_{1} .
\end{aligned}
$$

The integral equation for $\widetilde{\boldsymbol{C}}_{1}(q, \omega)$ can be written in the same way. These integral equations can be solved iteratively. The first iterates of $\tilde{M}(\omega)$ and $\widetilde{C}_{\perp}(q, \omega)$ are given by (ignoring the $\omega=0$ component)

$$
\tilde{M}_{1}(\omega)=\left(H_{0} / 4 \omega\right)[\delta(\omega+\Omega)-\delta(\omega-\Omega)],
$$

and

$$
\widetilde{C}_{1_{1}}(q, \omega)=0
$$

This is equivalent to a high-frequency approximation. This can be seen by equating the high-frequency terms on either side of Eq. (13). Therefore, in the first iteration $\tilde{M}(\omega)$ is nonzero only for $\omega=\Omega$; higher-order iterates are smaller than $\tilde{M}_{1}(\omega)$ in the $\Omega \rightarrow \infty$ limit. Therefore, in the large- $\Omega$ limit, $M(t)$ has the same frequency as $H(t)$ but is phase shifted. This gives an elliptic hysteresis loop in the upper half of the $M-H$ plane. The area of the hysteresis loop is given by

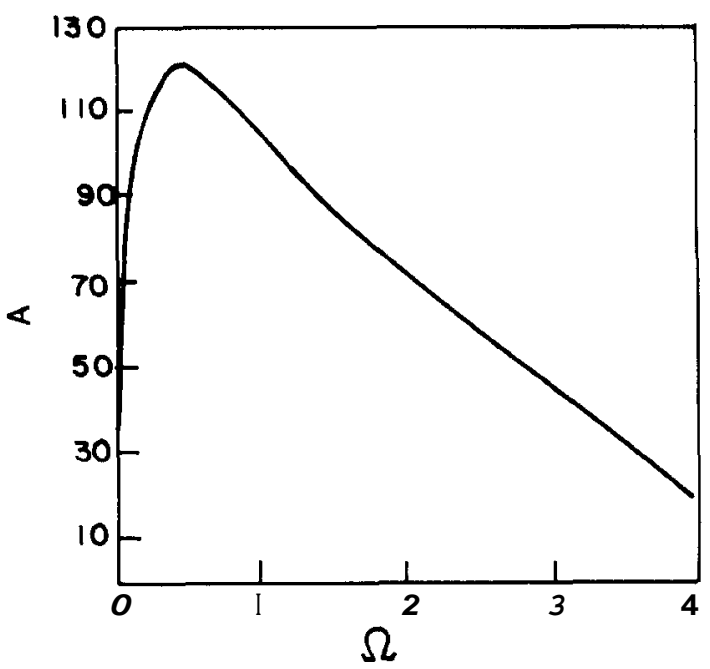

FIG. 17. Area of the hysteresis loop as a function of $\Omega . H_{0}$ is $10, \mathrm{r}=-0$, and $u=1$. The area of the hysteresis loop goes through a maximum as $\Omega$ increases. The initial rise follows $A \sim \Omega^{033}$

$$
\begin{aligned}
A & =\int_{0}^{2 \pi / \Omega} M(t) H_{0} \cos (\Omega t) d t \\
& =-\pi H_{0}^{2} / 8 \Omega .
\end{aligned}
$$

The negative sign denotes energy dissipation. A numerical evaluation of the area of the loop, for type-5 hysteresis loops, from Eqs. (7) show an $H_{0}^{2} / \Omega$ dependence (see Fig. 19).

\section{Temperaturedependence of the hysteresis loop}

The hysteresis loops of our model show a very strong dependence on the temperature. As the temperature decreases, the spatial fluctuations of the order parameter decrease. This makes the coercive field $H_{c}$ (and thus the area) larger. We restrict ourselves to type- 1 and type- 2 loops. Figure 20 shows the dependence of the area and the coercivity on the temperature, with $H, \Omega$, and $u$ fixed. We find the following fit to the data:

$$
\begin{aligned}
& A \propto r^{0.7}, \\
& H_{c} \propto r^{0.35} .
\end{aligned}
$$

Let us now keep $H_{0}$ fixed. As we increase R, we go from region 1 to 2 and so on. For higher temperatures, transitions from one type of loop to the next happen at higher values of $\Omega$. The loops get thinner (area decreases) as the temperature increases. Even when the temperature is larger than the critical temperature $\left(r>r_{c}\right.$ ) (so that the system is paramagnetic), hysteresis persists and the loops enclose a nonzero area. This is perhaps an artifact of the $N \rightarrow \infty$ limit. In a model with comparable fluctuations of the longitudinal and transverse components of the order parameter, we expect hysteresis loops to be absent for $r>r$. Our Monte Carlo results for the Ising model (Sec. III) support this view.

As we have seen earlier, the area of the hysteresis loop scales with $H_{0}$ and $\Omega$ for small $H_{0}$ and $\Omega$ in regions $1-3$ of the stability diagram Fig. 11. The exponents $\mathbf{a}$ and $\beta$ that characterize this scaling are independent of temperature. We have checked this for $r=-10$ and -2 .

\section{E. Mean-field theory}

In the mean-field approximation, all fluctuations of the order parameter are neglected. Therefore, any n-point correlation function can be written as a product of $n$ 

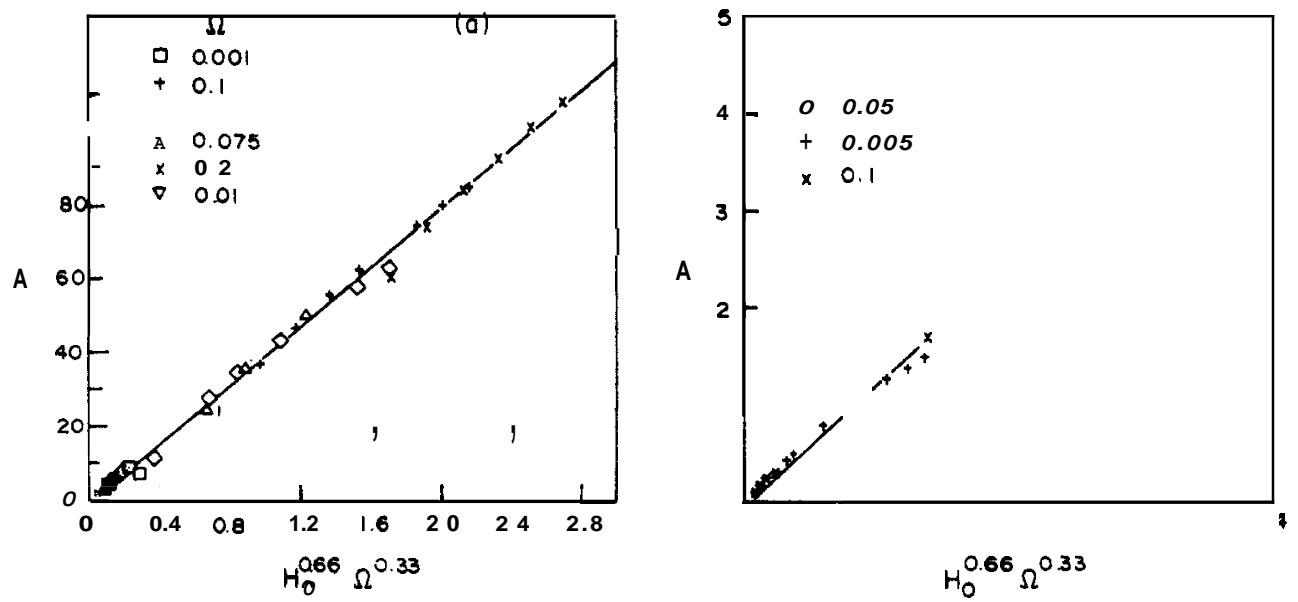

FIG. 18. A scaling plot which demonstrates that the area A of the hysteresis loop scales as A $\sim \boldsymbol{H}_{0}^{0.66} \Omega^{0.33}$. Data are given for the six values of $\Omega$ shown; the values of $\boldsymbol{H}_{0}$ are such that all points in this figure lie in regions 1,2 , or 3 of Fig. 11 . The exponents are independent of temperature. This is displayed in (a) $r=-10$ and (b) $\mathrm{r}=\mathbf{- 2}$.

one-point averages (the averages are taken over the probability distribution of the noise $\eta$ ). Under this approximation, the equation for the first moment $\left\langle\Phi_{a}\right\rangle$ is

$$
\begin{aligned}
\frac{d\left\langle\Phi_{\alpha}(\mathbf{x}, t)\right\rangle}{d t}=-\Gamma[ & \left(-\nabla^{2}+r\right)\left\langle\Phi_{\alpha}(\mathbf{x}, t)\right\rangle \\
& +u\left\langle\Phi_{\alpha}(\mathbf{x}, t)\right\rangle^{3} \\
& \left.-H_{0} \sin (\Omega t) \delta_{\alpha, 1}\right] .
\end{aligned}
$$

At $t=0$, the system is taken to be in equilibrium at

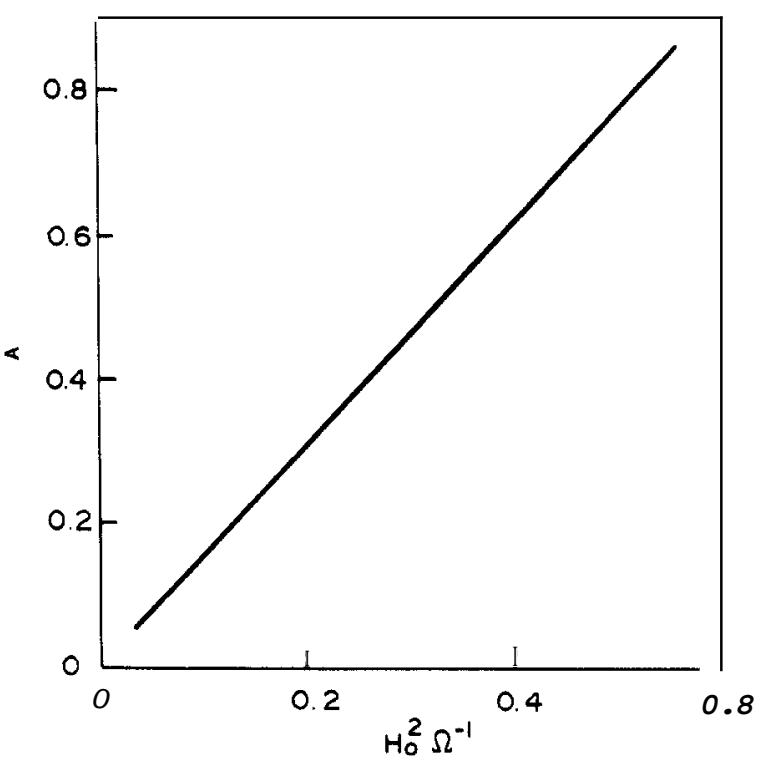

FIG. 19. Scaling of the area of the hysteresis loop for high frequencies as $\mathrm{A} \sim H_{0}^{2} \Omega^{-1}$ obtained from numerical integration of Eqs. (7) in agreement with Eq. (15).
$H_{\alpha}=(0+) \delta_{\alpha, 1}$ and $\mathrm{T}<T_{c}^{\mathrm{MF}}$ (ferromagnetic phase), i.e., $\mathrm{r}\left\langle 0\right.$. We therefore define $\left\langle\Phi_{(}(\mathbf{x})\right\rangle$ as the magnetization $M$ which is equal to $(-r / u)^{1 / 2}$. Moreover, $\left\langle\Phi_{a}(x)\right\rangle=0$ for all $\alpha \neq 1$. Since there are no spatial fluctuations of the order parameter and since the initial magnetization is homogeneous, the magnetization at subsequent times will also be homogeneous. Moreover, $\left\langle\Phi_{a}(\mathbf{x}, t)\right\rangle=0$ for all times when $\alpha \neq 1$. We measure time in units of $(2 \Gamma)^{-1}$. Therefore, the equation to be solved is

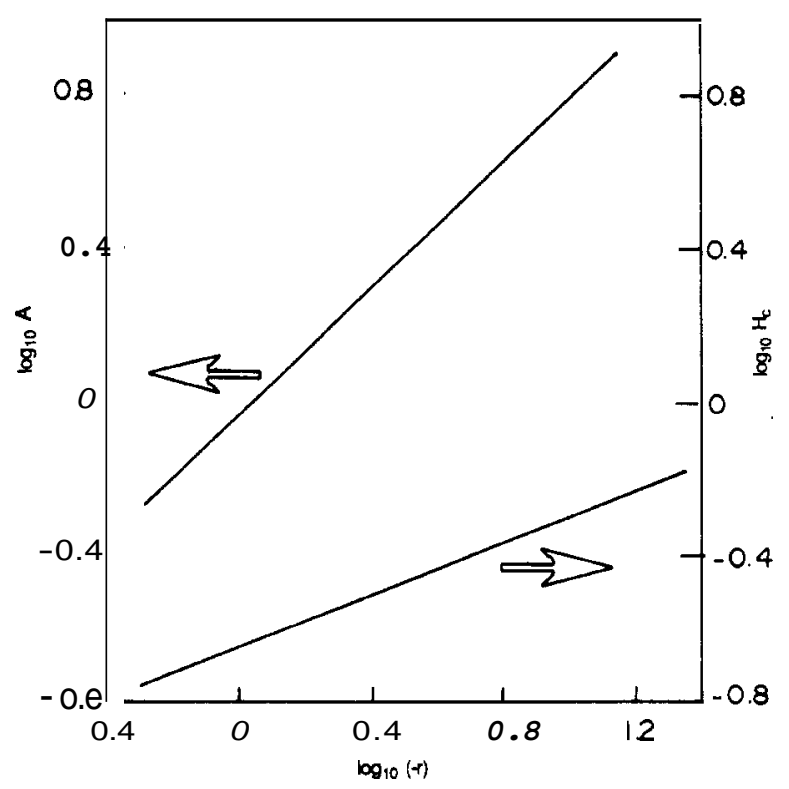

FIG. 20. Area and coercivity as a function of temperature keeping $H_{0}$ and $\Omega$ fixed (we restrict ourselves to regions 1,2 , and 3 of Fig. 11 , here $\Omega=0,01, H_{0}=1$ ). 


$$
\frac{d M(t)}{d t}=-\frac{1}{2}\left[r M(t)+u M^{3}(t)-H_{0} \sin (\Omega t)\right] .
$$

This nonlinear equation cannot be solved analytically for arbitrary $H$, and $\Omega$. We have to solve this equation for $M(t)$ using numerical methods. Accordingly we use an Euler finite-difference method to solve the differential equation. We also use a Runge-Kutta method to cross check the numerical results obtained by the Euler method. We plot the magnetization against the magnetic field for various $H_{0}$ and $\Omega$ to obtain hysteresis loops.

The main result of this section is the following: In the mean-field approximation there is no symmetric hysteresis $^{14}$ when the magnetic field amplitude is smaller than the spinodal field $H_{\text {sp }}$. Hysteretic behavior is seen only when the field amplitude $H_{0}$ reaches a value $H_{M}$ which is larger than $H_{\text {sp }} ; \boldsymbol{H}_{\boldsymbol{M}}$ is a monotonically increasing function of the frequency $\Omega$.

The evolution of hysteresis loops in the mean-field approximation is illustrated in Fig. 21. Here the frequency $\Omega$, the temperature $r$, and the coupling constant $u$ are kept fixed while the amplitude $H_{0}$ is increased. We see that for $H,<12.2=H_{M}$, the $M-H$ curve does not open out into a loop [Fig. 21(a)]. As soon as $H_{0}>12.2$, symmetric hysteresis loops are obtained [Fig. 21(b)]. These loops are squarish and the magnetization does not saturate. On a further increase of the amplitude, we observe that the loop saturates. If the amplitude is increased further, the area of the loop increases; however, no qualitatively new behavior is observed. ${ }^{13}$

As mentioned above, in the mean-field approximation symmetric hysteresis is obtained for $H_{0}>H_{M} ; H_{M}$ depends on the frequency of the applied field. For $\Omega=0.01$, $H_{M}$ is found to be equal to 12.2. When the frequency $\Omega=0$,

$$
H_{M}=H_{\mathrm{sp}}=\left(-4 r^{3} / 27 u\right)^{1 / 2}=12.17
$$

for $r=-10$ and $u=1$. The molecular field in this model can be calculated as follows:

$$
H_{\mathrm{mf}}=6 J M_{\text {eq }},
$$

where we have assumed a cubic lattice in three dimensions (the coordination number is 6). For $J=1, r=-10$, and $u=1$, we find that $H_{\mathrm{mi}}=18.954$. We thus see that $H_{M}$, the amplitude of the field required for magnetization

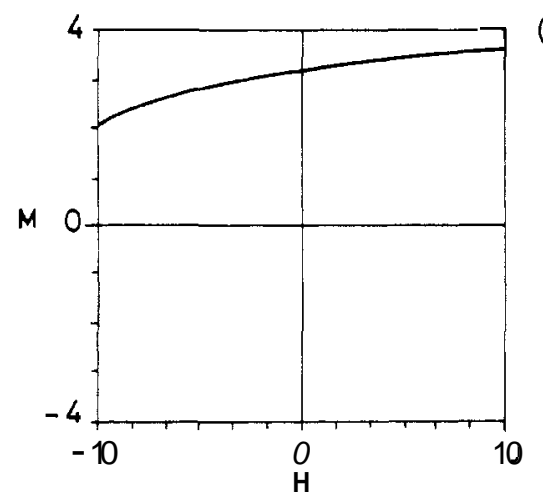

(a)
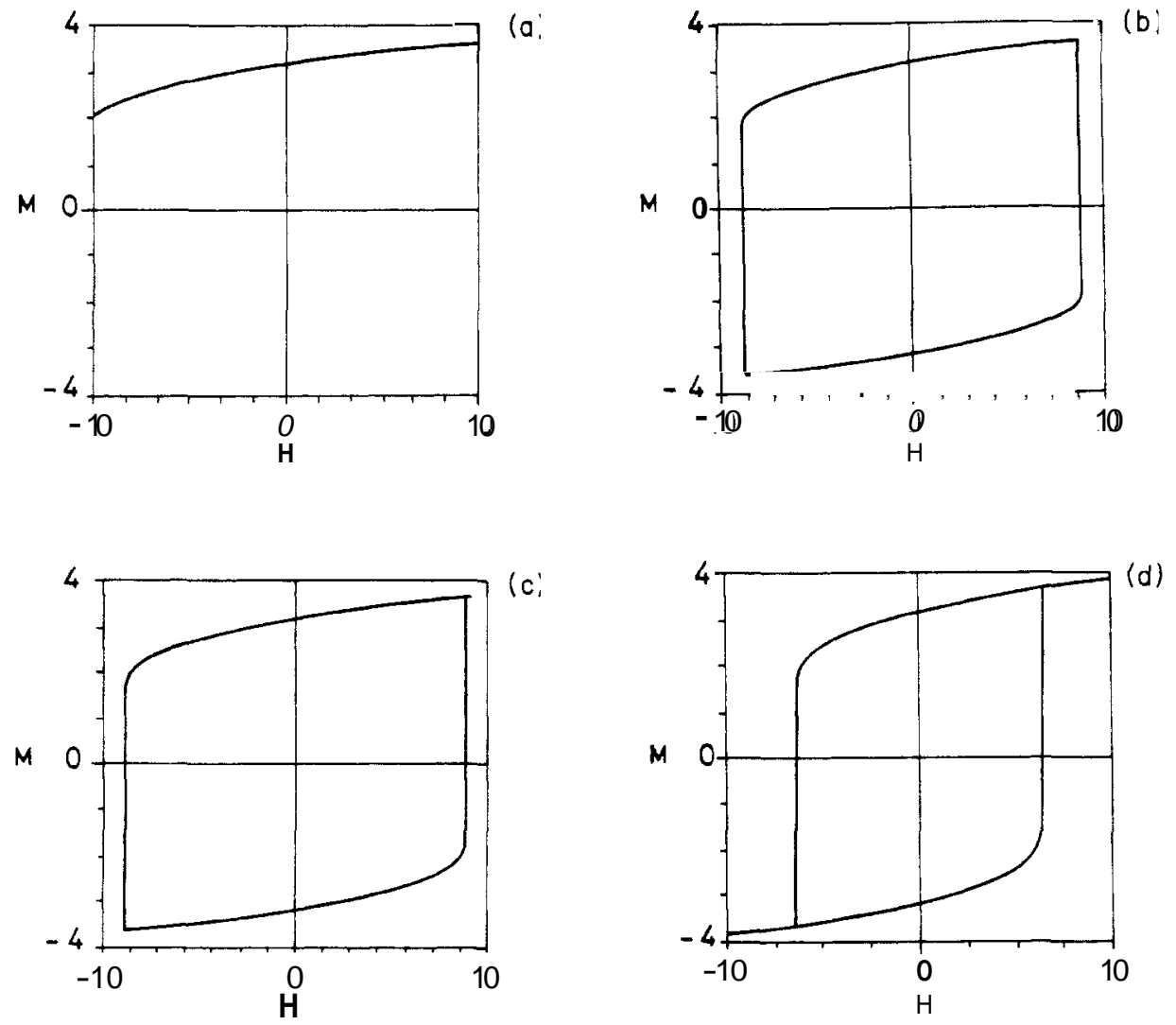

FIG. 21. Mean-field hysteresis loops obtained in the $\left(\Phi^{2}\right)^{2}$ theory. The amplitude of the field is different in each of these loops while the frequency is held constant at $\Omega=0.01$ : (a) $H_{0}=12$, (b) $H_{0}=12.25$, (c) $H_{0}=13$, and (d) $H_{0}=20$. For $H_{0}<12.2$, there is no symmetric hysteresis loop within the mean-field theory $(r=-10, \mathbf{u}=1)$. 
reversal is of the order of the molecular field. In real magnets this corresponds to a very large field $\left(H_{\mathrm{mf}} \approx 10^{7}\right.$ Oe). Therefore, we see that in a mean-field theory, symmetric hysteresis is observed only when $H_{0}$ is of the order of $H_{\mathrm{mr}}$. Such large fields are not attainable in laboratory experiments. Therefore, we conclude that a mean-field theory is an incorrect description of the hysteresis observed in laboratory magnets.

We can understand this mean-field behavior by a mechanical analogy. Consider a particle moving in a potential of the form

$$
V(x)=a x^{2}+b x^{4}
$$

where $a<0$ and $\boldsymbol{b}>0$. This potential has two symmetric minima at $x_{0}^{ \pm t}= \pm(-a / b)^{1 / 2}$. At $t=0$, let the particle be at $\boldsymbol{x}=\boldsymbol{x}_{0}^{+}$. This corresponds to a positive magnetization $M=+M_{\text {eq }}$ in the ferromagnet. Let us now apply a homogeneous periodic field which couples linearly to $\boldsymbol{x}$ :

$$
V(x, t)=a x^{2}+b x^{4}-x H_{0} \sin (\Omega t) .
$$

For small $H_{0}\left(<H_{\text {sp }}\right)$, the particle will remain in the $x_{0}^{+}$ minimum. This corresponds to an absence of hysteresis. When $H_{0} \geq H_{\mathrm{sp}}$, the $\boldsymbol{x}_{0}^{+}$minimum disappears. The particle rolls down the potential well into the $\boldsymbol{x}_{0}^{-}$minimum. This corresponds to symmetric hysteresis. However, the particle takes a time $\tau_{R}$ to roll down the potential well. If the frequency of the field is larger than the inverse roll-down time $1 / \tau_{R}$, then, by the time the particle has reached the bottom ( $x_{0}^{-}$minimum), the $x_{0}^{+}$minimum reappears and the particle remains at $\boldsymbol{x}=x_{0}^{+} \cdot \tau_{R}$ decreases as $H_{0}$ increases and, therefore, for larger frequencies, a larger amplitude is required to obtain symmetric hysteresis.

In our mean-field theory, the shapes of hysteresis loops have a nontrivial frequency dependence for $H_{0}>H_{M}(\Omega)$. Keeping $H_{0}$ fixed and greater than $H_{M}(\Omega)$, we monitor the shape of the loops as a function of $\Omega$. For small $\Omega$, the loops are symmetric and spindle-shaped with the magnetization having attained saturation (Fig. 22). As $\Omega$ increases, the loop does not saturate, but is still symmetric (the particle reaches the $x_{0}^{-}$minimum but does not settle down in it). Further increase in $\Omega$ rotates the symmetric loop until its major axis is along the $H$ axis. For very large $\Omega$, the loop is entirely in the upper half of the $M-H$ plane. It is no longer symmetric (the particle does not have enough time to reach the $x_{0}^{-}$minimum). The loop in the upper half plane drifts slowly downwards for high $\Omega$ and for very high $\Omega$, the loop forms a closed curve with very little enclosed area.

\section{F. Response to pulsed magnetic fields}

To understand the dynamical processes involved in the hysteresis problem, we study the response of the spin system to a pulsed magnetic field of the form

$$
H_{\alpha}(t)=H_{0} \Theta(t) \delta_{\alpha, j},
$$

where $\theta(t)=1$ for $t<0$ and -1 for $t>0$. The order parameter $\Phi_{\alpha}(\mathbf{x}, t)$ evolves in time as specified by the Langevin equation (3). The final dynamical equations for the magnetization and the transverse correlation function in the $N \rightarrow \infty$ limit' are obtained by replacing $H_{0} \sin (\Omega t)$ in Eq. (7) by $H_{0} \Theta(t)$. At $t=0$, the system is in equilibrium at $H=H_{0}$. Therefore, the initial conditions to be used in Eq. (7) are the equilibrium values of $\boldsymbol{M}$ and $C_{1}$ at $H=H_{0}$ and temperature $\boldsymbol{r}\left(<r_{c}\right)$.

The differential equations have to be solved numerically to obtain $M(t)$ versus $t$ for various values of $H_{0}$ (keeping $r$ and $\boldsymbol{u}$ fixed). The equations describe the decay of magnetization towards its equilibrium value for $\boldsymbol{H}=-H_{0}$. A typical magnetization decay curve is plotted in Fig. 23 for $H_{0}=1.0$. The time evolution of $S(t)$, the momentum integral of the transverse correlation function, for the same values of parameters is also shown in Fig. 23. Note that $S(t)$ has a maximum when the magnetization is zero. The magnetization curve has three distinct portions: (i) the initial exponential decay from $M(t=0)$ to some intermediate value, (ii) the plateau region, where $M(t)$ is nearly a constant, and (iii) the final exponential decay towards the equilibrium magnetization at $H=-H_{0}$, which is $-M(t=0)$. There are thus three distinct time scales $\tau_{1}, \tau_{2}$, and $\tau_{3}$ which can be extracted from the magnetization decay curve (see Fig. 23). These
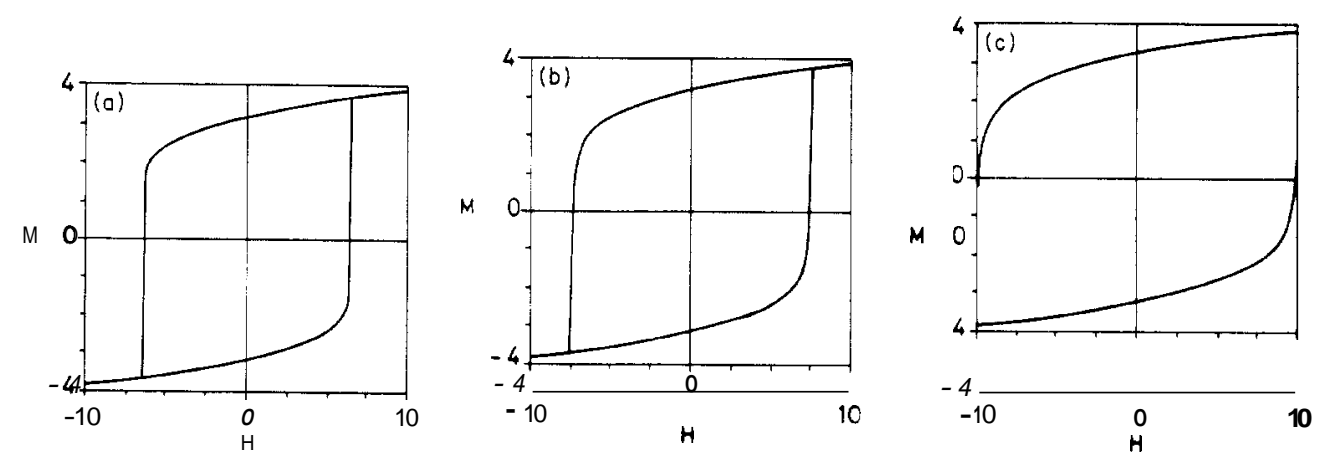

FIG. 22. Mean-field hysteresis loops. $H_{0}\left(>H_{M}\right)$ is fixed at 20 . The loops are plotted for various values of $\mathrm{R}:(\mathrm{a}) \Omega=0.01,(\mathrm{~b})$ $\Omega=0.1$, and $(\mathrm{c}) \Omega=1(r=-10, u=1)$. 


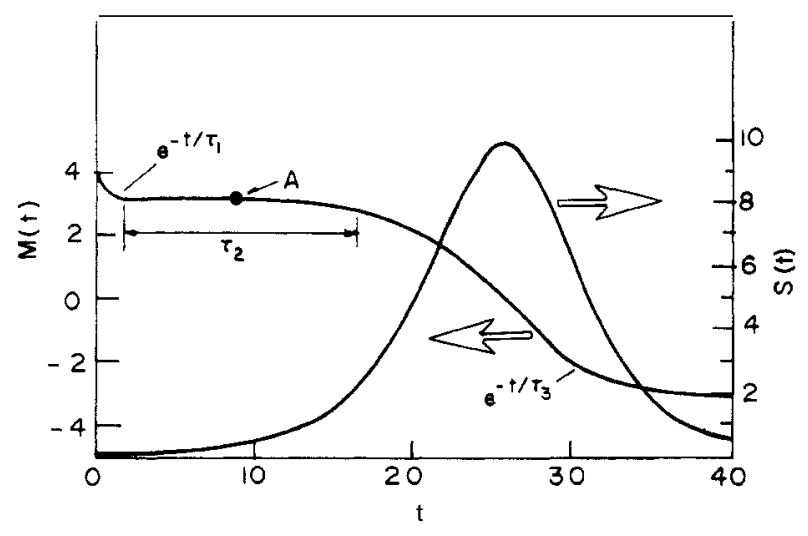

FIG. 23. Magnetization reversal in response to a pulsed magnetic field. Note the existence of three time scales: the initial exponential decay, the intermediate magnetization plateau, and the final exponential decay. The integrated transverse correlation function $S$ is drawn in the same graph.

are related to three distinct dynamical processes of the spin system in response to a time-varying magnetic field. We will use the mechanical analogy discussed in Sec. II E with a slight extension to illustrate this relation. The extension consists in subjecting the particle to a fluctuating force which allows it to escape over the barrier in between the two minima of the free-energy functions (Fig. 24). This mechanical picture resembles the singlecomponent $\Phi^{4}$ theory. This is not strictly true since there exist spatial fluctuations of the order parameter in the $\Phi^{4}$ theory which are absent in the mechanical analogue. At $t<0$, the situation is as shown in Fig. 24(a) - the field $H_{0}$ is positive and the particle is in the $x_{0}^{+}$minimum. At $t=0$, the field is suddenly flipped to $-H_{0}$. The particle does not respond at once, only the potential changes $\left(x_{0}^{-}\right.$ is now the stable minimum and the $x_{0}^{+}$metastable minimum has shifted towards the origin). The particle initially. relaxes towards the $\mathbf{x}_{0}^{+}$metastable minimum. This relaxation time is 7 ,. The particle has to decay to the $x_{0}^{-}$global minimum eventually. It does so under the action of the fluctuating force which kicks the particle over the barrier Fig. 24(b). The time scale associated with this hopping over the barrier is $\tau_{2}$. The particle now relaxes towards the $x_{0} \overrightarrow{ }$ minimum (after having hopped over the barrier), Fig. 24(c). This relaxation time is $\tau_{3}$. These are the dynamical processes associated with the decay of the particle from the metastable minimum to the stable minimum. The process described above is for a single-component order parameter with no spatial fluctuations. Let us, in the same spirit, extend the mechanical analogy to the $\mathrm{N}$-component order-parameter theory $(N=\infty)$. Here, the particle does not hop over the barrier. The potential in this case has an infinite set of degenerate minima when $H_{0}=0$. When the field is flipped from $+H_{0}$ to $-H_{0}$, the particle moves from the metastable minimum to the stable minimum along the intervening minima (transverse modes of decay). This roll-down time is $\tau_{2}$ (transverse motion along the intervening minima). Since the radius of the trajectory in coordinate space, $\Sigma_{\alpha} x_{\alpha}^{2}$ [corresponding to $\sum_{\alpha=1}^{N} \Phi_{\alpha} \Phi_{\alpha}$ in the orderparameter space] is infinite (in the $N \rightarrow \infty$ limit), the projection of the position along the $\alpha=1$ direction remains essentially constant for some time (analogous to the magnetization plateau in Fig. 23).

We now describe how we operationally define these three time scales $\tau_{1}, \tau_{2}$, and $\tau_{3}$. Consider point $A$ in Fig. 23. This point is defined as the point $\left(t_{1}, M\left(t_{1}\right)\right)$ at which $d^{2} M / d t^{2}=0 \quad\left[0<t_{1}<t_{0}\right.$, where $t_{0}$ is such that $\left.M\left(t_{0}\right)=0\right]$. In the interval $\left(0, t_{1}\right)$, we fit the magnetization-decay curve to an exponential $\mathrm{e}^{-t / \tau_{1}}$. We thus determine the time scale $\tau_{1} . \tau_{2}$ is merely given by $t_{0}-t_{1}$. In the interval $\left(t_{0}, t_{2}\right)$, where $t_{2}$ is such that $M\left(t_{2}\right)=-M(t=0)$ (to an accuracy of $10^{-8}$ ), we fit the magnetization curve to another exponential $\mathrm{e}^{-t / \tau_{3}}$. This determines the time scale $\tau_{3}$. The magnetization decay obviously depends on the value of $\mathrm{H}_{0}$ - the larger the magnitude of the magnetic-field flip, the faster is the decay. We thus ask for the dependence of the time scales $\tau_{1}, \tau_{2}$, and $\tau_{3}$ on $H_{0}$ (for a fixed $r$ and $u$ ). This is shown in Fig. 25 for $r=-10$ and $u=1$. The magnetic field $H_{0}$
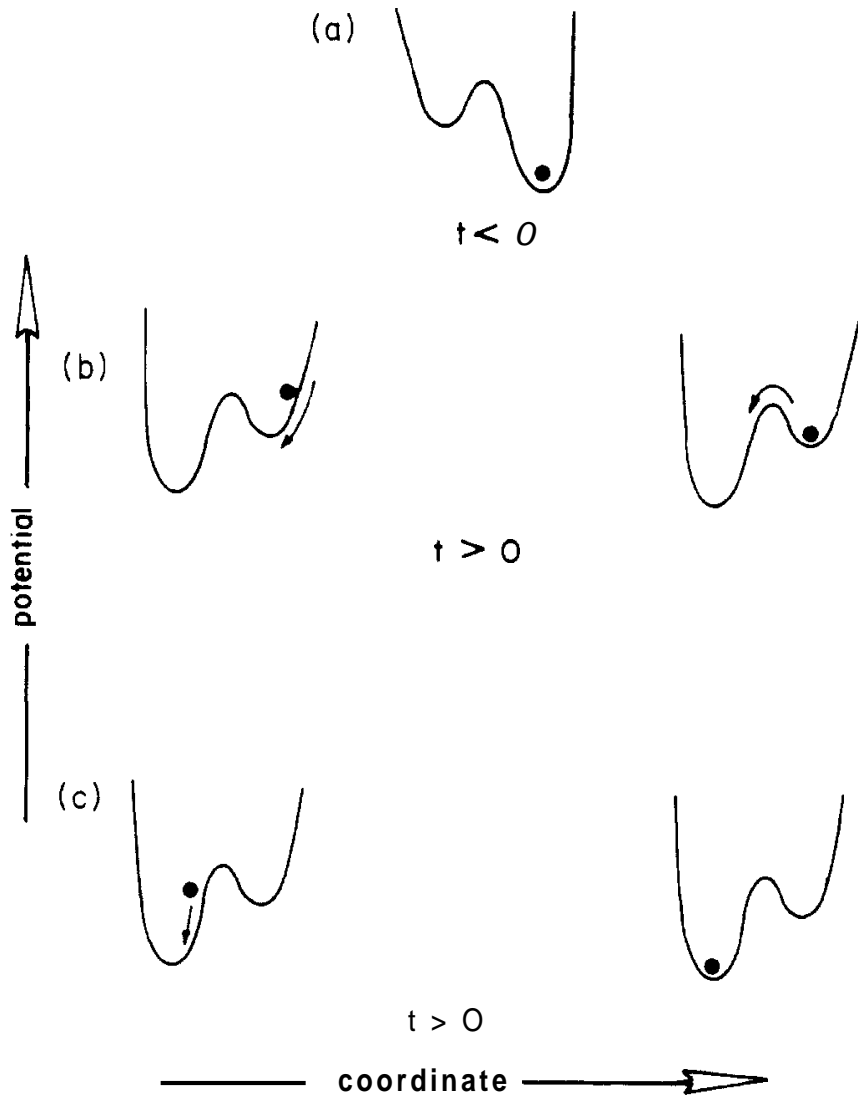

FIG. 24. Mechanical analogy to describe the three time scales associated with magnetization reversal. (a) $t<0$, particle is in the $x_{0}^{-}$minimum; (b) $t>0$, particle decays to the $x_{0}^{+}$ minimum of the potential in a time $\tau_{1}$ and then attempts to hop over the barrier; and (c) after a time $\tau_{2}$ it crosses the barrier and then decays to the $x_{n}{ }^{-}$minimum in a time $\tau_{3}$. 


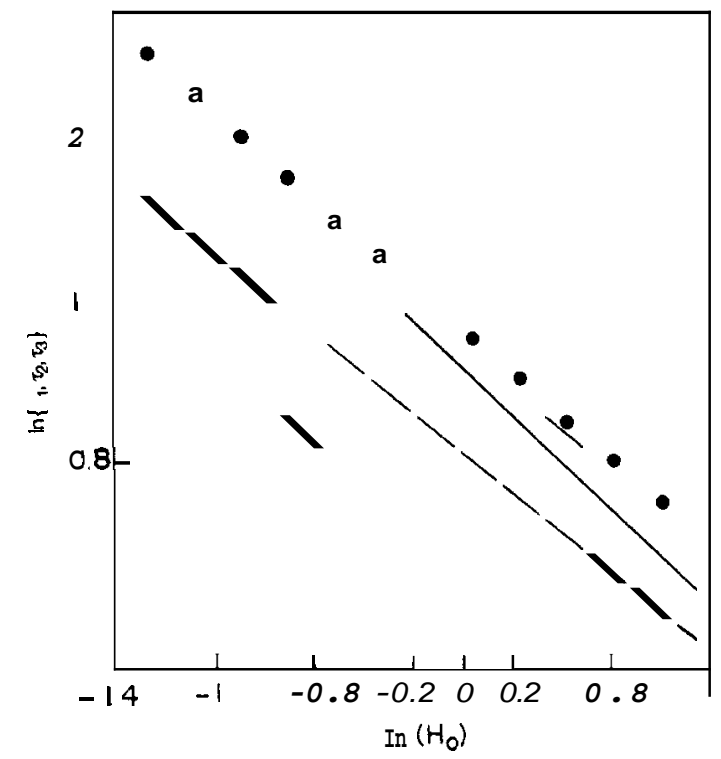

FIG. 25. The power-law dependence of the three time scales on the magnetic field $H_{0}$. The solid line denotes $\tau_{1}$, the dotted line denotes $\tau_{2}$, and the dashed line denotes $\tau_{3}$ (here $r=-10$, $u=1$ ).

ranges between 10 and 0.01 . From the figure we conclude that $\tau_{1}\left(H_{0}\right) \propto H_{0}^{-1.4 \pm 0.1}, \quad \tau_{2}\left(H_{0}\right) \propto H_{0}^{-1.1 \pm 0.1}, \quad$ and $\tau_{3}\left(H_{0}\right) \propto H_{0}^{-1.0 \pm 0004}$.

The mechanical analogy, generalized to $\mathrm{N}$ components, gives us a way of understanding the variation of $\tau_{2}$ on $H$, When the field is flipped, the particle rolls down the intervening minima with a velocity $v$. The kinetic energy $\frac{1}{2} v^{2}$ should balance the potential-energy difference, $2 x_{0}^{+} H_{0}$. For small $H_{0}, x_{0} \propto H_{0}$ (linear response). ${ }^{15}$ Therefore, $v \propto H_{0}$, i.e., the velocity of the particle is proportional to the potential $H_{0}$. Since $v \propto \tau^{-1}$, the inverse of a time scale in the mechanical problem, we see that $\tau \propto H_{0}^{-1}$. The time scale $\tau$ in this N-component mechanical picture is analogous to $\tau_{2}$ in the $\left(\boldsymbol{\Phi}^{2}\right)^{2}$ theory. The $H_{0}^{-1}$ dependence is consistent with the observations in ferrites (though the mode of magnetization decay is quite different).

Let us try to relate then time scales $\tau_{1}, \tau_{2}$, and $\tau_{3}$ to the hysteresis window of Shenoy and Agarwal. ${ }^{16}$ The Shenoy-Agarwal bounds for the hysteresis window entail two time scales: the local relaxation time in the metastable minimum and the first-passage time to go from the metastable minimum to the stable minimum. In our notations,

$$
\tau_{1}<\Omega^{-1}<\tau_{2}+\tau_{3} .
$$

According to Shenoy and Agarwal, for frequencies outside this window, "good" hysteresis (conventional squarish loops) is not observed. We see from our analysis that hysteresis is observed even for frequencies outside the window. Note, however, that the magnetic field in our analysis is sinusoidal ( $|d H / d t|$ is therefore not constant), while in the Shenoy-Agarwal treatment it is a periodic ramp function (where $|d H / d t|$ is a constant almost everywhere).

We compute $\ln \left(\tau_{i}^{-1}\right)$ versus $H_{0}$ (for $i=1,2,3$ ) and compare the resulting power-law dependences with the boundaries which separate the five qualitatively different hysteretic behavior. Such a comparison is not without ambiguity since these boundaries (especially those between regions 4 and 5 and regions 4 and 3) are computed using arbitrary criteria. Nevertheless, we note that the $\tau_{1}$ curve approximately follows the boundary between regions 2 and 3 , the $\tau_{2}$ curve approximately follows the boundary between regions 3 and $\mathbf{4}$, and the $\tau_{3}$ curve is nearly coincident with the boundary between regions 1 and 2 .

\section{HYSTERESIS IN THE ISING MODEL}

The last section dealt with magnetic hysteresis in a continuous spin system. In this section, we discuss magnetic hysteresis in a lattice model of a discrete spin system. In particular, we study the response of the twodimensional Ising model with nearest-neighbor ferromagnetic interactions to a time-varying magnetic field. This response is studied by a Monte Carlo simulation using the algorithm of Metropolis et al. ${ }^{17} \mathrm{We}$ obtain hysteresis loops for a wide range of amplitudes and frequencies of the applied field and temperature on a square lattice.

The Hamiltonian of the two-dimensional Ising model we study is

$$
\mathscr{H}=-J \sum_{\langle i j\rangle} S_{i} S_{j}-H(t) \sum_{i} S_{l},
$$

where the spins $S_{i}(= \pm 1)$ occupy the sites $i$ of a square lattice and $\langle i j\rangle$ are nearest-neighbor pairs of sites. We set the scale of the energy by choosing the exchange coupling $J=1$. The magnetic field $H(t)$ varies periodically in time. We use periodic boundary conditions for the spins. We have done simulations on lattices of size $10 \times 10,20 \times 20$, and $50 \times 50$.

We use the standard Metropolis Monte Carlo algorithm ${ }^{17,18}$ which does not conserve the order parameter. We update the spin variables $S_{i}$ by stepping sequentially through the lattice.

At time $t=0$, the spin system is assumed to be in equilibrium at a temperature $T$ below the ordering temperature $T_{c}$ and magnetic field $H_{0}, T_{c}$ is obtained from the exact Onsager result $^{19}$ for the two-dimensional Ising model on a square lattice

$$
T_{c}=-2 / \ln (1-\sqrt{2}) .
$$

The spin system is allowed to equilibrate at a temperature $T\left(<\boldsymbol{T}\right.$, ) and magnetic field $H_{0}$. We start with all spins $S_{i}=\operatorname{sgn}\left(H_{0}\right)$. We visit each site on the lattice sequentially and update the spins using the algorithm mentioned before. We thus "sweep" across the entire lattice. We go through several such "sweeps" and evaluate the magnetization and energy every fifth sweep. We go through the lattice 2000 times and check whether the magnetization and energy have equilibrated. 


\section{A. Monte Carlo simulation in the study of hysteresis}

Once the initial equilibrium configuration is established, we study the response of the Ising system to a time-varying magnetic field. For numerical convenience, the magnetic field evolves periodically in a steplike linear fashion (Fig. 26). From the figure we see that there are two time scales [measured in Monte Carlo steps (MCS)] associated with the magnetic field: one is $T$, , the interval of time over which the field is a constant, and the other is $\tau_{2}$, the period of the magnetic field. The magnetization is evaluated at every $\tau_{3}$ th pass over the lattice (where $\tau_{3}<\tau_{1}$ and $\tau_{1} / \tau_{3}$ is an integer). The magnetization evolves periodically under the influence of the periodic magnetic field. We allow the magnetization to evolve for 20 cycles of the magnetic field without collecting any data. This is to allow for enough time for the transients to settle down. We then collect data for the magnetization and plot the magnetization versus the magnetic field. Since the period $\tau_{2}$ is much smaller than the recurrence time of the random number generator (which for the VAX $11 / 730$ is $2^{31}-1$ ), the magnetization at time $t$ and $t+\tau_{2}$ are affected by different random numbers. Therefore, the hysteresis loops generated after each cycle are not identical to each other. We thus average over several periods (we have taken averages over up to 1000 periods). This produces an average hysteresis loop (Fig. 27). We ask for the deviation of the loop from its average. This is obtained by computing the standard deviation of the magnetization from its average value (taken over 1000 periods, say). To make these statements more explicit let us define $M_{a}(H(t))$ to be the magnetization as a function of the field $H(t)$ during the ath cycle ( $\mathrm{a}$ is the loop index). Then the average magnetization and the standard deviation are given by

$$
M_{L}(H(t))=\frac{1}{L} \sum_{a=1}^{L} M_{a}(H(t)),
$$

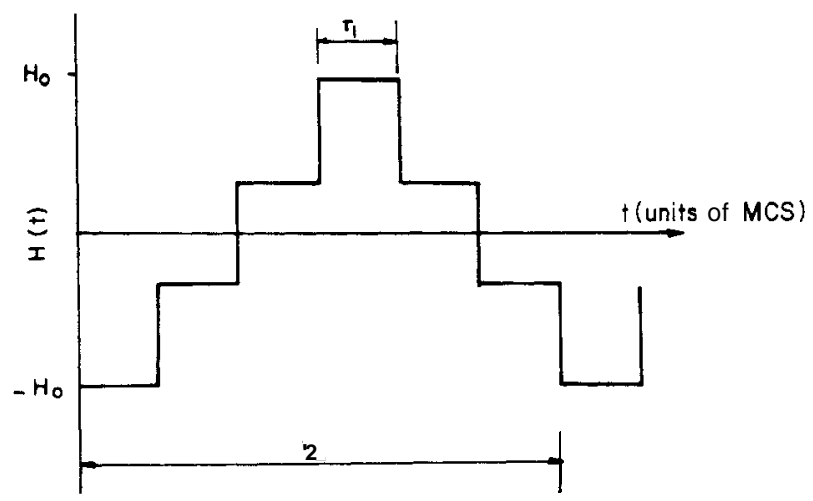

FIG. 26. Periodic applied magnetic field. Note the existence of the two time scales $\tau_{1}$ and $\tau_{2} . H_{0}$ is the amplitude of the magnetic field.

$$
\sigma_{L}(H(t))=\frac{1}{L} \sum_{a=1}^{L}\left[M_{a}(H(t))-M_{L}(H(t))\right]^{2},
$$

where $\boldsymbol{L}$ is the total number of loops over which we have averaged and $t$ is measured modulo $\tau_{2}, M_{L}$ and $\sigma_{L}$ are computed iteratively as the loops are traversed.

The standard deviations of the magnetization at different points on the average hysteresis loop are represented by error bars (Fig. 27). We see that the error bars are large (in some cases, they are of the order of the maximum value that the magnetization takes for a given frequency and amplitude of the field) in the regions where the magnetization jumps from a negative (positive) value to a positive (negative) value. The error bars do not seem to decrease with an increase in the number of loops over which we perform the average. We have checked this by computing the standard deviations when the number of loops over which we average range from 80 to 1000 . There is, however, a strong dependence on the size of the system - the errors decrease with increasing lattice size. For example, when the temperature is 1.8 , the amplitude $H_{0}=2$ and the frequency such that the loop is of type 1 , the standard deviation in the region of the loop where the magnetization changes sign is 0.025 for a $50 \times 50$ lattice and 0.48069 for a $10 \times 10$ lattice. Since the errors involved are so large, any quantitative measurement like the area of the hysteresis loop becomes meaningless for small lattice sizes. We now present the qualitative results obtained from our simulation studies in detail.

\section{B. Results of the Monte Carlo simulation}

\section{Variation with the frequency of the field}

We study the changes in the shape of the hysteresis loop as we vary the two time scales $\tau_{1}$ and $\tau_{2}$ (see Fig. 26), keeping the temperature $T$, the lattice size $N$, and the am-

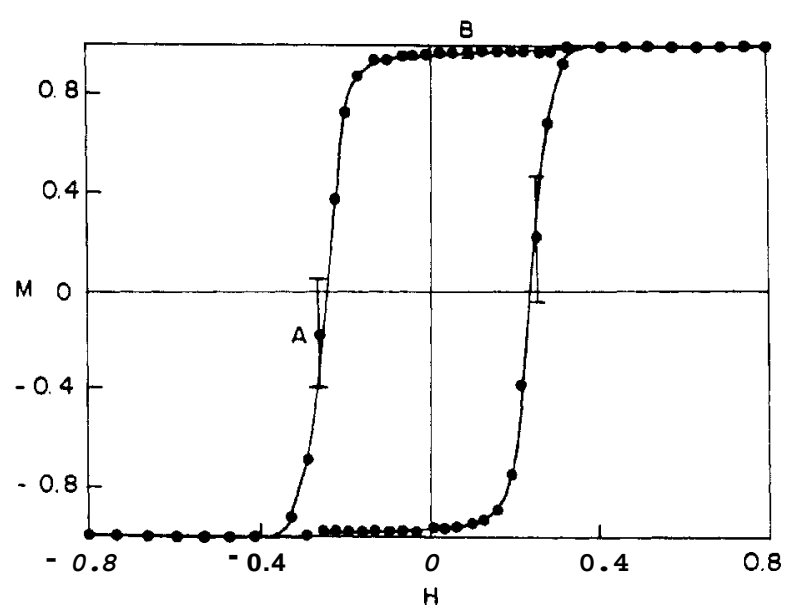

FIG. 27. Typical average hysteresis loop obtained on a $20 \times 20$ lattice. The average has been taken over 880 cycles of the magnetic field. The temperature is $1.8, \tau_{1}=15, \tau_{2}=1500$, and $H_{0}=0.8$. The error bars are large in region $A(\sigma=0.5713)$ and extremely small in region $B\left(\sigma=0.2 \times 10^{-4}\right)$. 
plitude of the field $\boldsymbol{H}$, constant. When $\tau_{1}$ and $\tau_{2}$ are small, the magnetic field changes rapidly. We call such a variation a high-frequency $(\Omega)$ variation. When $\tau_{1}$ and $\tau_{2}$ are large, the changes in the field are slow and we refer to low-frequency variations. For high frequencies (the precise values depend on $\boldsymbol{N}, T$, and $H_{0}$ ), the $\boldsymbol{M} \cdot \boldsymbol{H}$ curve is entirely in the upper half-plane ${ }^{20}$ [type 5, Fig. 28(a)]. The drift of the ellipse towards the $H$ axis (type 4) cannot be resolved in our Monte Carlo simulation because of the large errors involved. For lower frequencies, the loops are roughly elliptical with their major axes along the $M=0$ line [type 3, Fig. 28(b)]. A decrease in the frequency of the field rotates the major axis so that it is inclined to the $\boldsymbol{H}$ axis [type 2, Fig. 28(c)]. For lower frequencies, the hysteresis loop is squarish with a change in the sign of the curvature at the tips indicating saturation. A further decrease produces squarish hysteresis loops [type 1, Fig. $28(\mathrm{~d})]$ with the magnetization saturating at $M=1$. When $\Omega$ is very large $\left(\tau_{1}, \tau_{2}=2\right)$, the hysteresis loop collapses onto a straight line (Fig. 29) parallel to the $\boldsymbol{H}$ axis (this corresponds to the $\Omega \rightarrow \infty \boldsymbol{M} \cdot \boldsymbol{H}$ curve discussed in the Introduction). We compute the coercive field $\boldsymbol{H}$, by a naive interpolation routine, In loops of type 1 , an increase in $\Omega$ increases the coercivity [Figs. 30(a) and 30(b)] and hence the area. This is because the phase lag of the magnetization with respect to the magnetic field increases as a function of $\Omega$. Therefore, a larger field is required for magnetization reversal. The same behavior is observed in the $\left(\boldsymbol{\Phi}^{2}\right)^{2}$ model and in the experimental studies of Ewing and co-workers. ${ }^{5}$

Consider loops of type 1 , with fixed $H_{0}$ and $T$. From the Monte Carlo data we notice that the same coercive field $H_{c}$ (and hence the area) is obtained with a higher frequency $\Omega$ and larger lattice $N$ as with a lower frequency and smaller lattice [Figs. 31 (a) and 3(b)], i.e., to get the same $H_{c}$, one requires a smaller frequency for a smaller lattice size. This implies that $H_{c}$ !and the area) does not depend on $\Omega$ or $N$ separately but on some ratio of the two. ${ }^{21}$
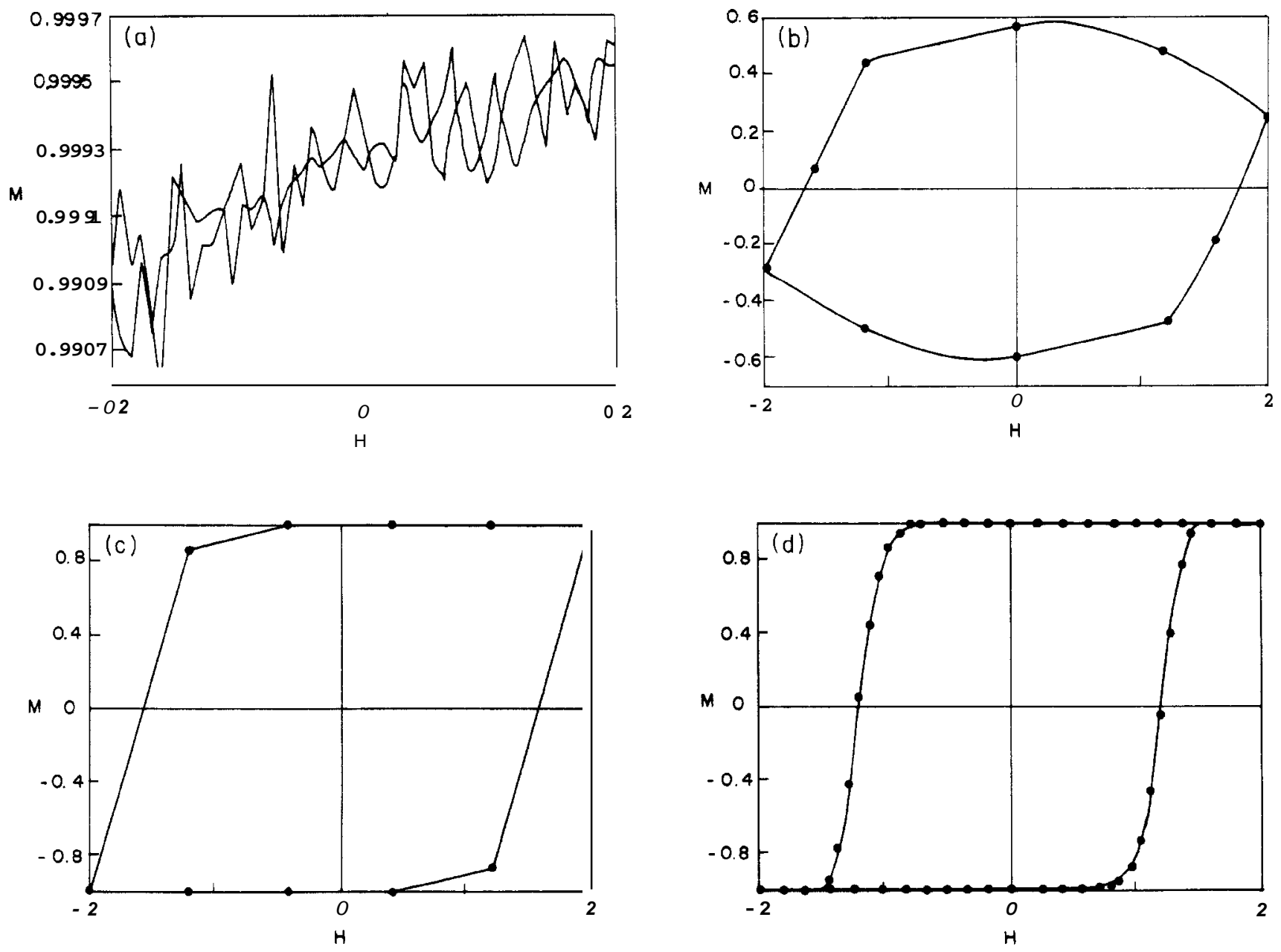

FIG. 28. The average hysteresis loop as a function of $\Omega(N=10$ and $T=1)$ : (a) $H_{0}=0.2, \tau_{1}=1, \tau_{2}=100$; (b) $H_{0}=2, \tau_{1}=11$, $\tau_{2}=10 ;$ (c) $H_{0}=2, \tau_{1}=6, \tau_{2}=60 ;$ (d) $H_{0}=2, \tau_{1}=6, \tau_{2}=600$. 

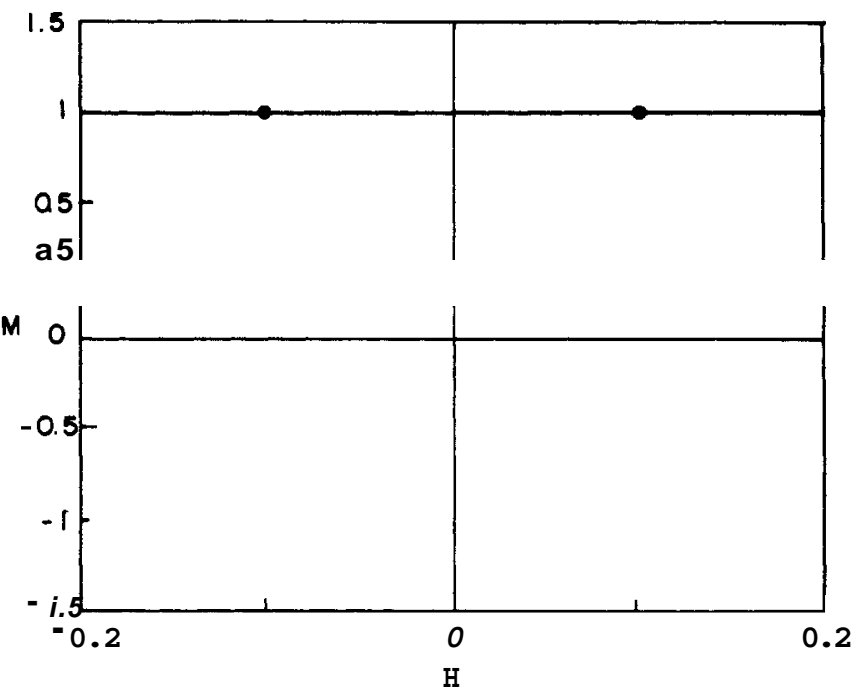

FIG. 29. M-H curve for $\tau=1, \tau=2$, and $\mathrm{H}=0.2, T=1$, and $N=10$. This corresponds to the $\Omega \rightarrow \infty$ limit of the hysteresis loop [see Fig. 3(b)].
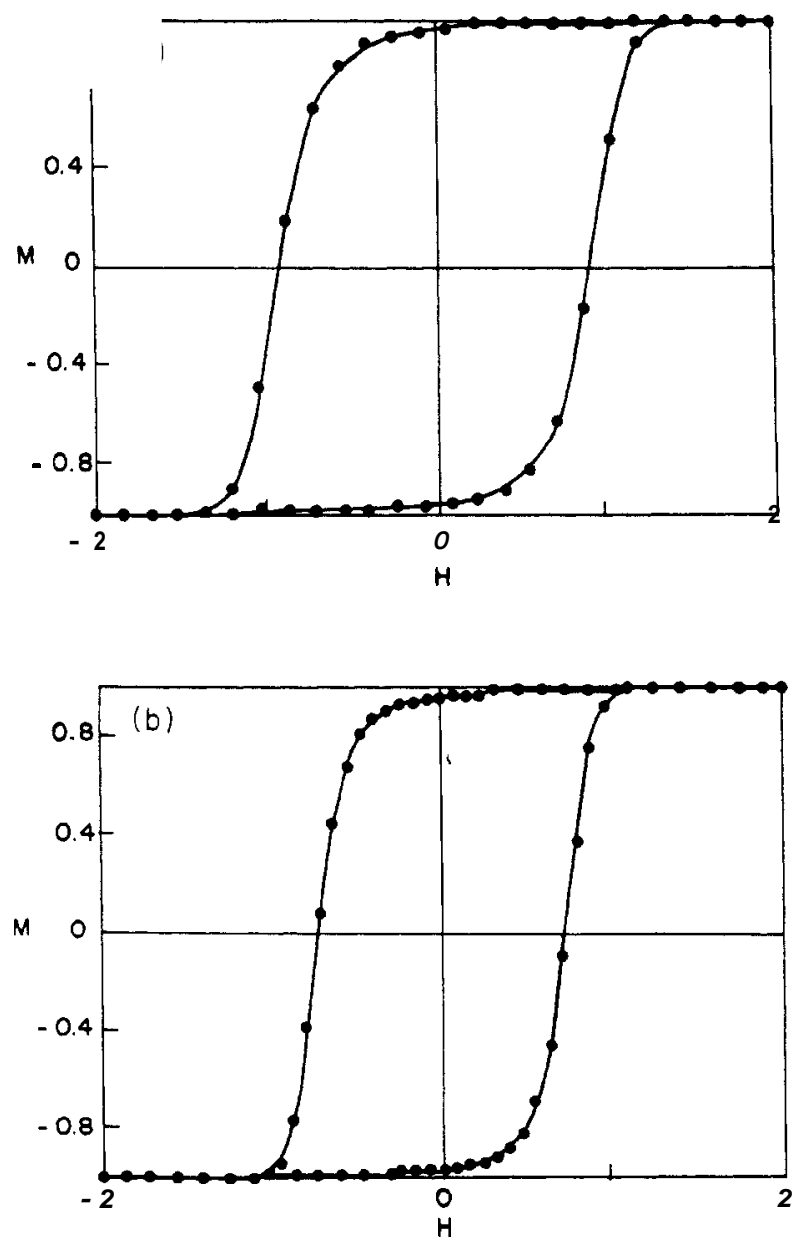

FIG. 30. Increase in the coercive field $H_{c}$ as $\Omega$ increases for type-1 hysteresis loops $\left(H_{0}=\mathbf{2}, \mathbf{T}=\mathbf{1 . 8}\right.$, and $\left.N=20\right)$ : (a) $\tau_{1}=1$, $\tau_{2}=50 ;(\mathrm{b}) \tau_{1}=1, \tau_{2}=100$.

\section{Variation with the amplitude of the field}

We study the amplitude $\left(H_{0}\right)$ variation of the hysteresis loop keeping the temperature $\mathrm{T}$ and the lattice size $\mathrm{N}$ fixed. Moreover, we hold $\tau_{1}$ and $\tau_{2}$ constant. As $H_{0}$ decreases, the loop evolves in a sequence opposite to that mentioned in Sec. III B, i.e., the loops change from types $1 \rightarrow 2 \rightarrow 3 \rightarrow 5$ (type 4 is again difficult to resolve).

We find that for hysteresis loops of type $1, H_{c}$ is of the order of the exchange coupling $J$. This is because the amplitude of the applied field is also of order $J$. If we apply a very small field, then the values of $\tau_{1}$ and $\tau_{2}$ required to obtain a type-1 loop are extremely large. The coercive fields in this case will be small.

From an analysis of the Monte Carlo data we see that the coercive field (and the area) do not depend on the frequency and the amplitude of the magnetic field separately but on some ratio of the two. ${ }^{21}$
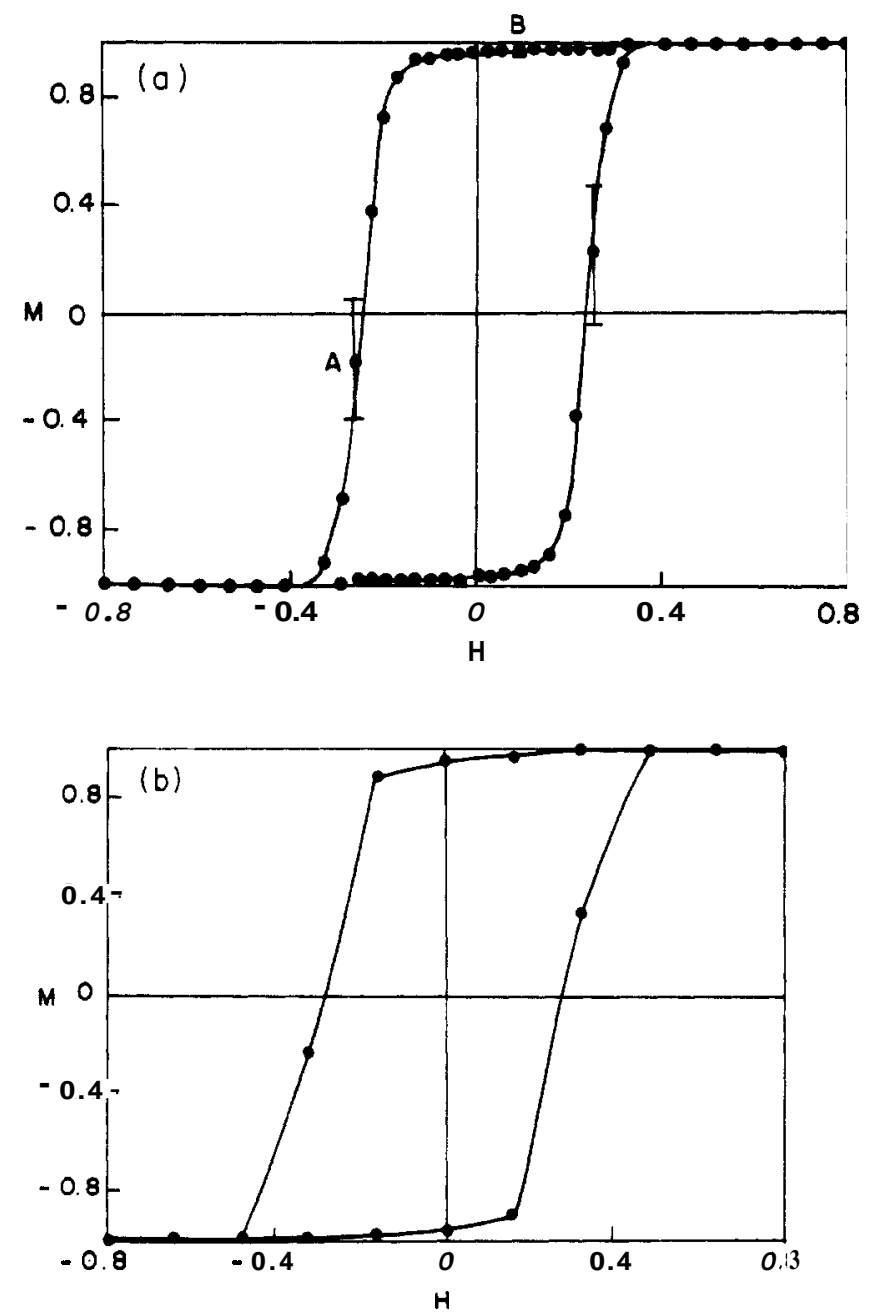

FIG. 31. The coercive field $H_{c}$ is the same $(=0.25)$ for $\left(T=1.8\right.$ and $\left.H_{0}=0.8\right):$ (a) $\tau_{1}=15, \tau_{2}=1500, N=20$ and (b) $\tau_{1}=25, \tau_{2}=500, N=50$. 


\section{Variation with temperature}

An increase in the temperature increases thermal fluctuations of the order parameter which hasten the decay of a metastable state. We thus see that an increase in temperature decreases the coercive field [Figs. 32(a) and 32(b)]. If we fix $H, \tau_{1}, \tau_{2}$, and $N$, a decrease in the temperature changes the shape of the loops from types $1 \rightarrow 2 \rightarrow 3 \rightarrow 4 \rightarrow 5$ (type 4 is difficult to resolve).

If we fix $H_{0}$ and $N$, we find from our Monte Carlo data that, to get the same $H$, (and hence the same area), the applied field must have a lower frequency at a lower temperature [Figs. 33(a) and 33(b)]. ${ }^{21}$

We see that the area of the hysteresis loop shrinks as the temperature increases until, for temperatures larger than the ordering temperature $T$, the area of the loop shrinks to zero and the magnetization follows the magnetic field. This is in contrast with the high-temperature behavior of the hysteresis loops in the $\mathrm{N}$-component $\left(\boldsymbol{\Phi}^{2}\right)^{2}$ model with $N=\infty$.
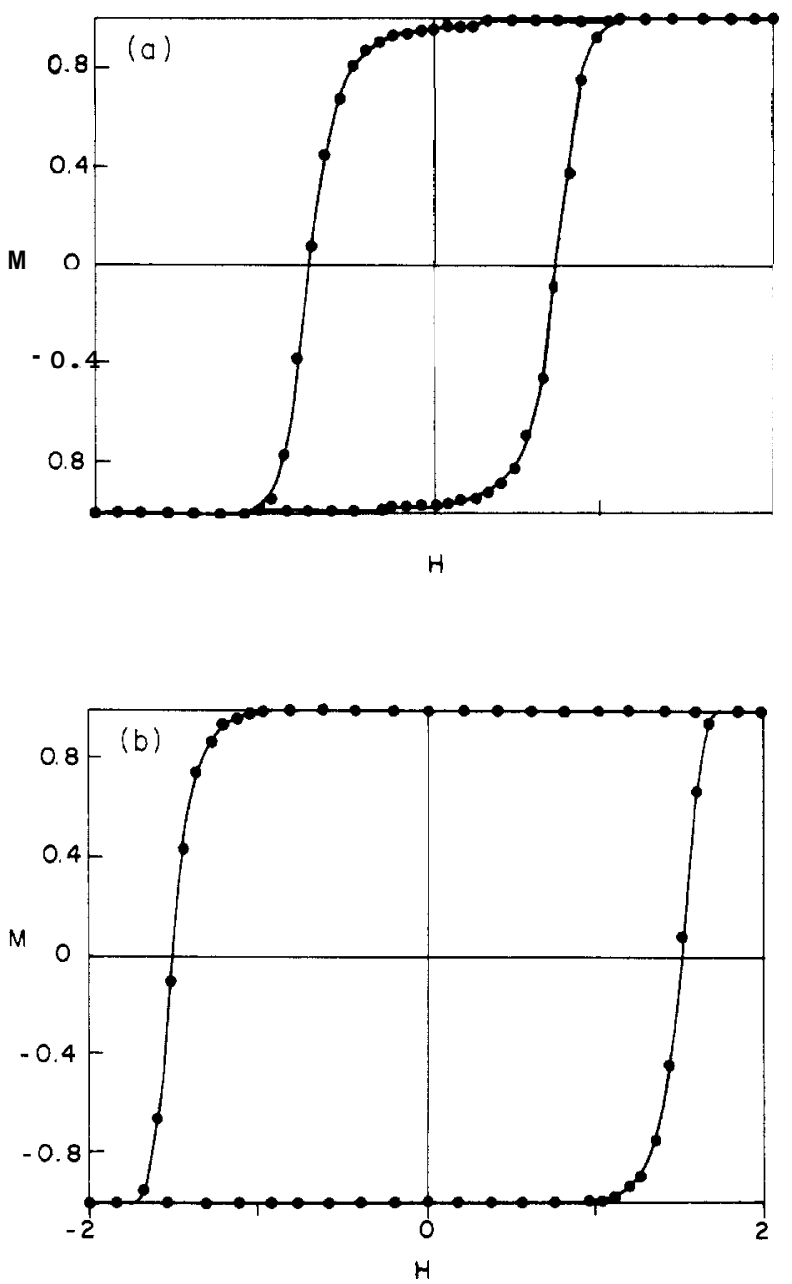

FIG. 32. Decrease in $H_{6}$ as a function of temperature ( $N=20, H_{0}=2, \tau_{1}$ and $\tau_{2}$ are held fixed): (a) $T=1.8$ and (b) $T=1$.

\section{Variation with lattice size}

We perform the Monte Carlo simulations on $10 \times 10$, $20 \times 20$, and $50 \times 50$ square lattices. We can therefore look for the size dependence of the hysteresis loops. We find that if $T, H_{0}$, and $\tau_{1}$ and $\tau_{2}$ are fixed, an increase in the lattice size changes the shape of the loops from types $5-4$ (difficult to resolve) $\rightarrow 3 \rightarrow 2 \rightarrow 1$ [Figs. 34(a) and 34(b)]. Keeping $\tau_{1}, \tau_{2}, H_{0}$, and $T$ a constant, a larger lattice gives a larger remnant magnetization (since there are more spins in a larger lattice) and a smaller coercivity. The dependence of $\boldsymbol{H}$, on the lattice size $\boldsymbol{N}$ is very weak. The qualitative dependence of $H_{c}$ on $\mathrm{N}$ agrees with experiments on $\mathrm{MnBi}$ grains. ${ }^{3}$

\section{Spin configurations}

It is interesting to study the behavior of the spins and the domains in a lattice as a function of the changing magnetic field. We have observed the spin configurations
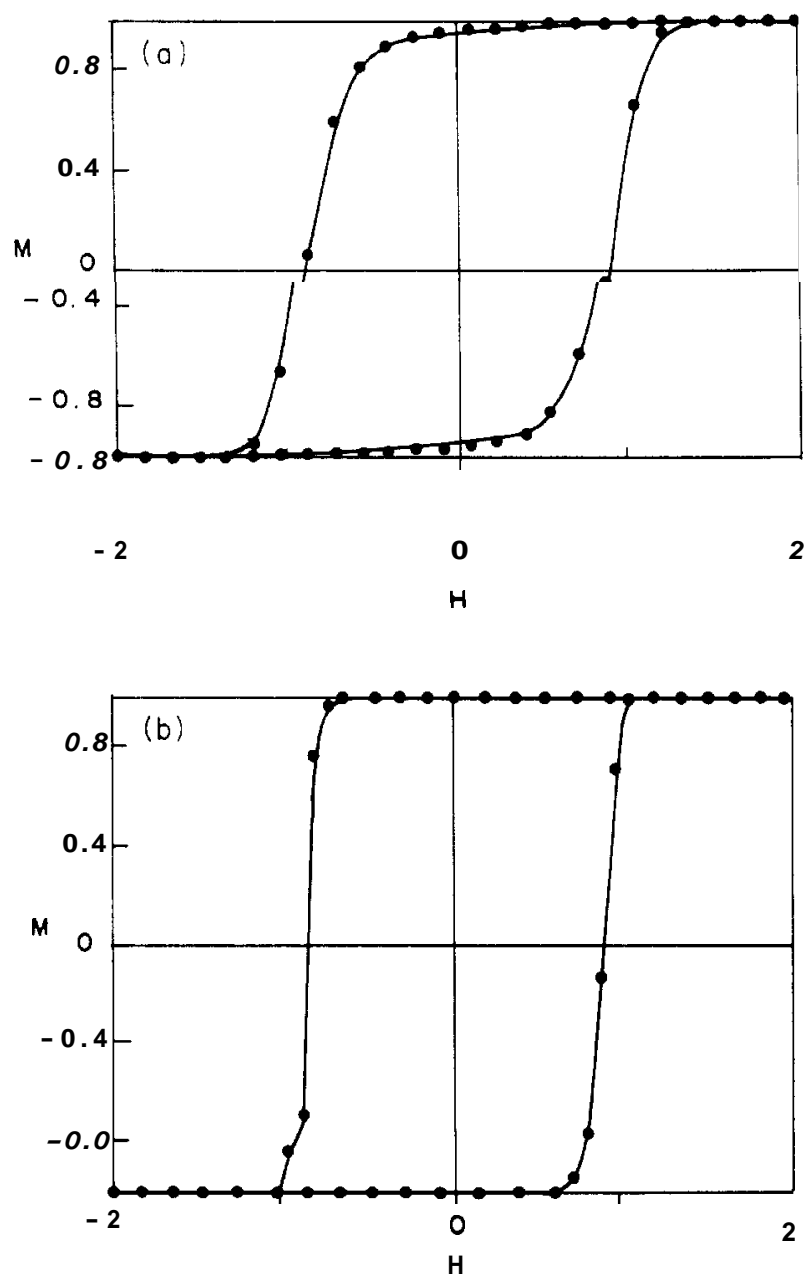

FIG. 33. The coercive field $H_{c}$ is the same $(=0.8)$ for $\left(T=1.8, H_{0}=2\right.$, and $\left.N=50\right)$; (a) $T=1.8, \tau_{1}=1, \tau_{2}=50$ and (b) $T=1, \tau_{1}=15, \tau_{2}=1500$. 
as a function of the magnetic field for the lattices under study. The spin configurations are such that the domains of up (down) spins in a matrix of down (up) spins are not circular and compact, but are ramified, especially in the segment of the $\boldsymbol{M} \cdot \boldsymbol{H}$ curve where the magnetization changes sign. A time sequence of these configurations [Figs. 35(a)-35(e)] show coalescence and coarsening of these domains. Thus, theories of hysteresis that concentrate on the nucleation of a droplet and not on its subsequent coarsening cannot be completely valid.

This ends our analysis of hysteresis in the twodimensional Ising model. The results we obtain are mainly qualitative but they corroborate the results obtained in Sec. 11. A more detailed quantitative study of the twoand three-dimensional models, especially of the hypothesized scaling relations (see Ref. 21) is required. These questions will be tackled in the future.
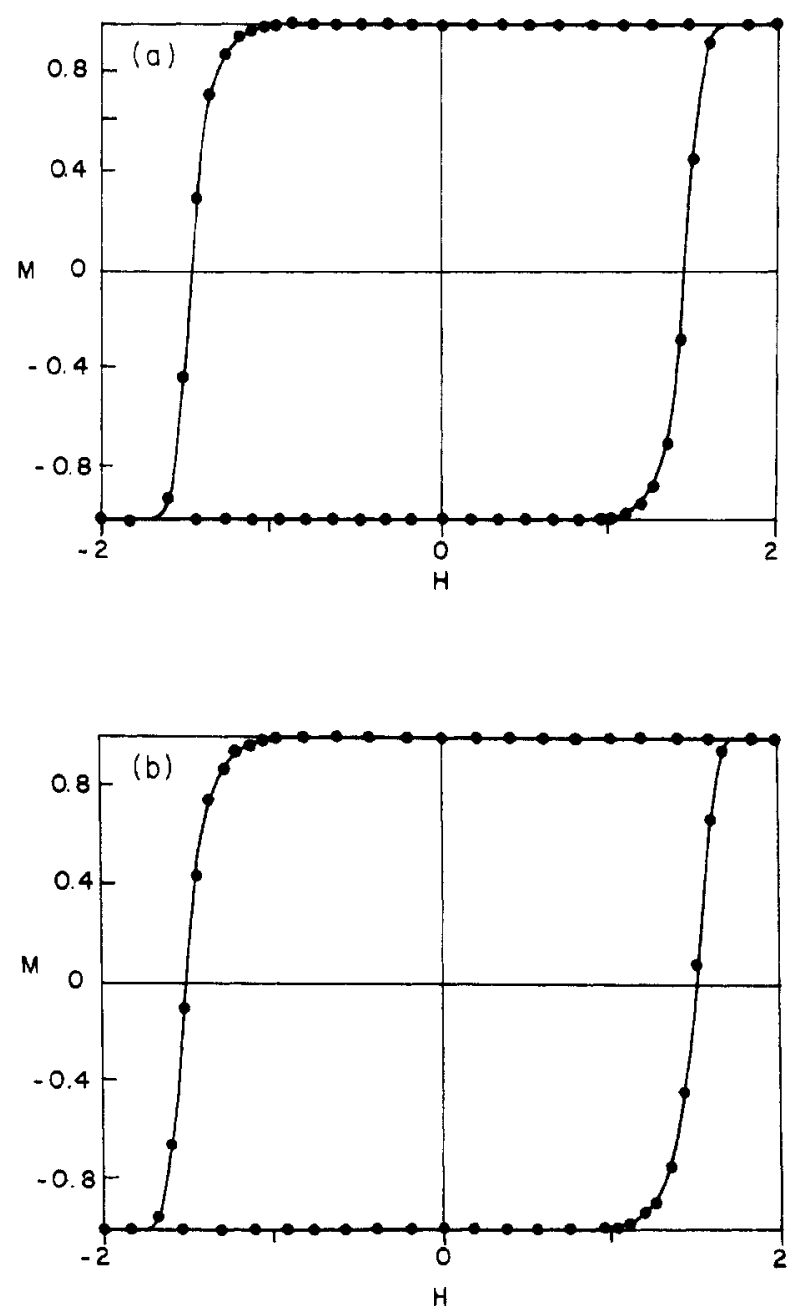

FIG. 34. Decrease in $H_{c}$ as a function of the lattice size $(T$, $H_{0}, \tau_{l}$, and $\tau_{2}$ are fixed): (a) $N=50$ and (b) $N=20$.

\section{COMPARISON WITH OTHER THEORIES OF HYSTERESIS}

In this section we compare the theory just presented with other theories of hysteresis. Other theories that have been constructed so far fall into three categories: (a) purely phenomenological theories such as those of Rayleigh, ${ }^{3,22}$ Preisach, ${ }^{23}$ and Hodgdon and Coleman, ${ }^{24}$ (b) theories that rely on the limit of metastability to precipitate hysteresis, such as the Stoner-Wohlfarth theory ${ }^{25,26}$ and the theory described by Landau and Lifshitz, ${ }^{8}$ and (c) theories that use first-passage time techniques, such as the theory of Shenoy-Agarwal. ${ }^{16}$ Some of the purely phenomenological theories obtain reasonable agreement with experimentally determined hysteresis loops at very low frequencies; however, these theories rely on various ad hoc assumptions and require several phenomenological parameters.

The Rayleigh theory ${ }^{3,22}$ recognizes that hysteresis cannot occur in linear-response theory and therefore allows the permeability to have the simplest nonlinear dependence on the field. The magnetic induction is assumed to depend quadratically on the magnetic field. This relation clearly violates time-reversal invariance and therefore its exact status is uncertain.

Hodgdon and Coleman ${ }^{24}$ have proposed a constitutive relation between the magnetic induction $B$ and the magnetic field $\mathrm{H}$ to describe rate-independent ferromagnetic hysteresis. Rate independent implies that the hysteresis loop and the area are independent of the frequency of the oscillating magnetic field. The theory assumes that there is no phase lag between $\boldsymbol{B}$ and $\mathrm{H}$ and further that there is no frequency dependence of the hysteresis loops. The nonanalyticity of various terms in the constitutive equation makes its derivation from a microscopic Langevin equation seem unlikely.

A popular phenomenological model is the Preisach model ${ }^{23}$ which assumes that a bulk magnetic material is a composite of small, single-domain, magnetic grains interacting with each other. Each particle is characterized by a magnetic moment and the coercive fields (positive and negative switching fields). Knowing the response of these individual grains, the response of the bulk sample is determined with the help of an experimentally measured (difficult to measure) or (most often) assumed distribution function (which is a function of the magnetic moments and the coercive fields). Consequently the Preisach model lacks predictive power.

All these theories are empirical fits to experimental data. In particular, the Hodgdon and Coleman theory and the Preisach model have too many free parameters and functions which have to be specified before a quantitative comparison with experimental observations can be made. The theory that we have constructed is a theory valid over length scales larger than the phonon-magnon mean free path and over time scales larger than the phonon-magnon relaxation time. This theory is to be contrasted with the phenomenological theories which are macroscopic theories and deal with macroscopic variables.

The Stoner-Wohlfarth theory ${ }^{25,26}$ investigates the hys- 
teretic behavior of a single-domain magnetic particle. The Landau-Lifshitz theory, ${ }^{8}$ on the other hand, provides a conceptual framework for analyzing hysteresis in a wider class of systems. The Stoner-Wohlfarth theory and the
Landau theory predict that hysteresis occurs whenever the metastable minimum of the free energy disappears. Both these theories picture the magnetization reversal in hysteresis as an equilibrium, mean-field process. These
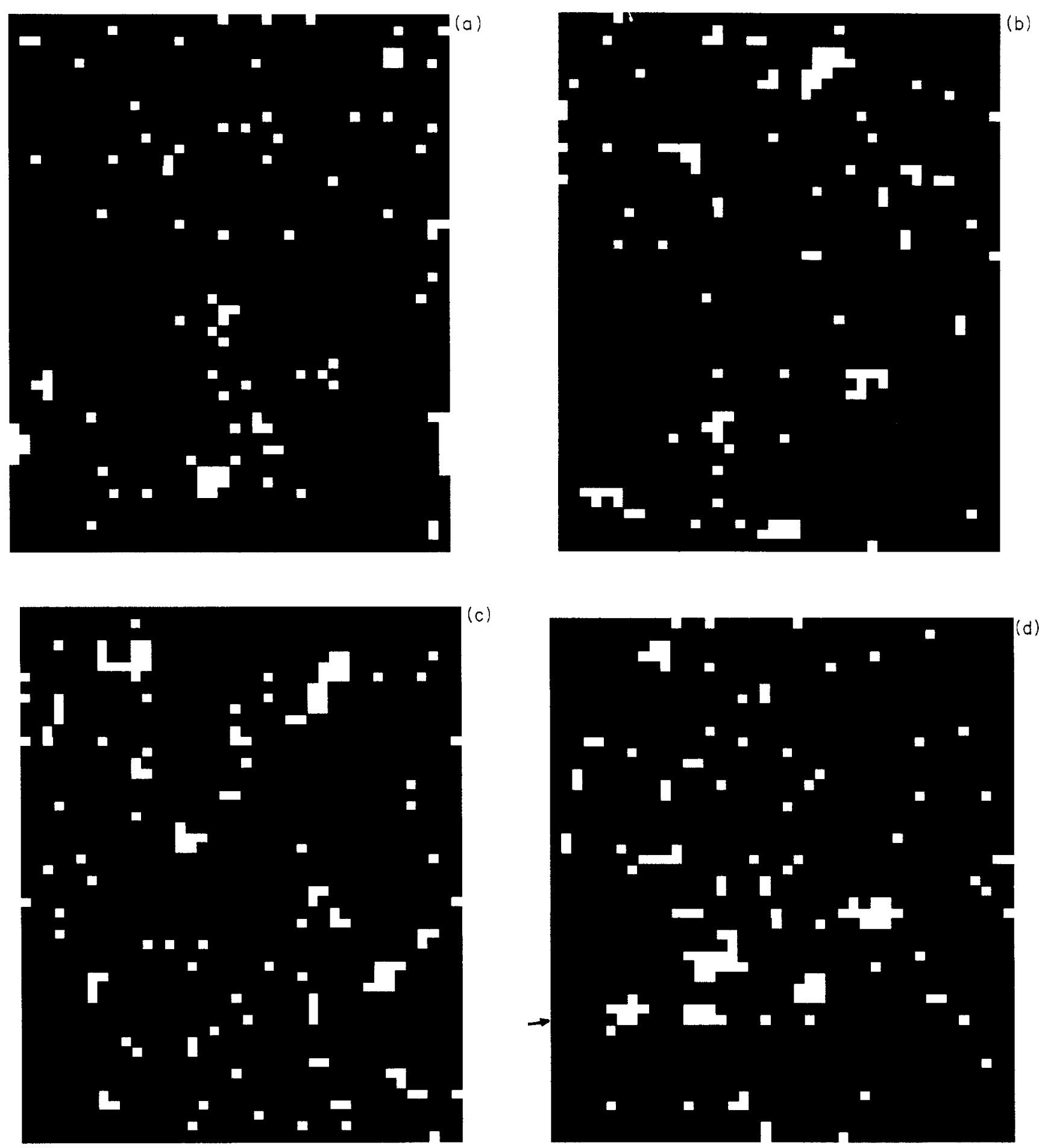

FIG. 35. Time sequence of spin configurations on a $50 \times 50$ lattice as a type- 1 hysteresis loop is traversed from $H=-H_{00}=-2$ to $\boldsymbol{H}=H_{0}=2$. Solid squares denote $S_{i}=-1$. Note that even when $H>0$, the spins are predominantly "down." (a) $H=-0.08$, (b) $H=-0.02$, (c) $H=0.02$, (d) $H=0.04$, (e) $H=0.08$, and (f) $H=0.16$. The cluster, marked by an arrow in (d) and (e), shows coarsening and coalescence. 

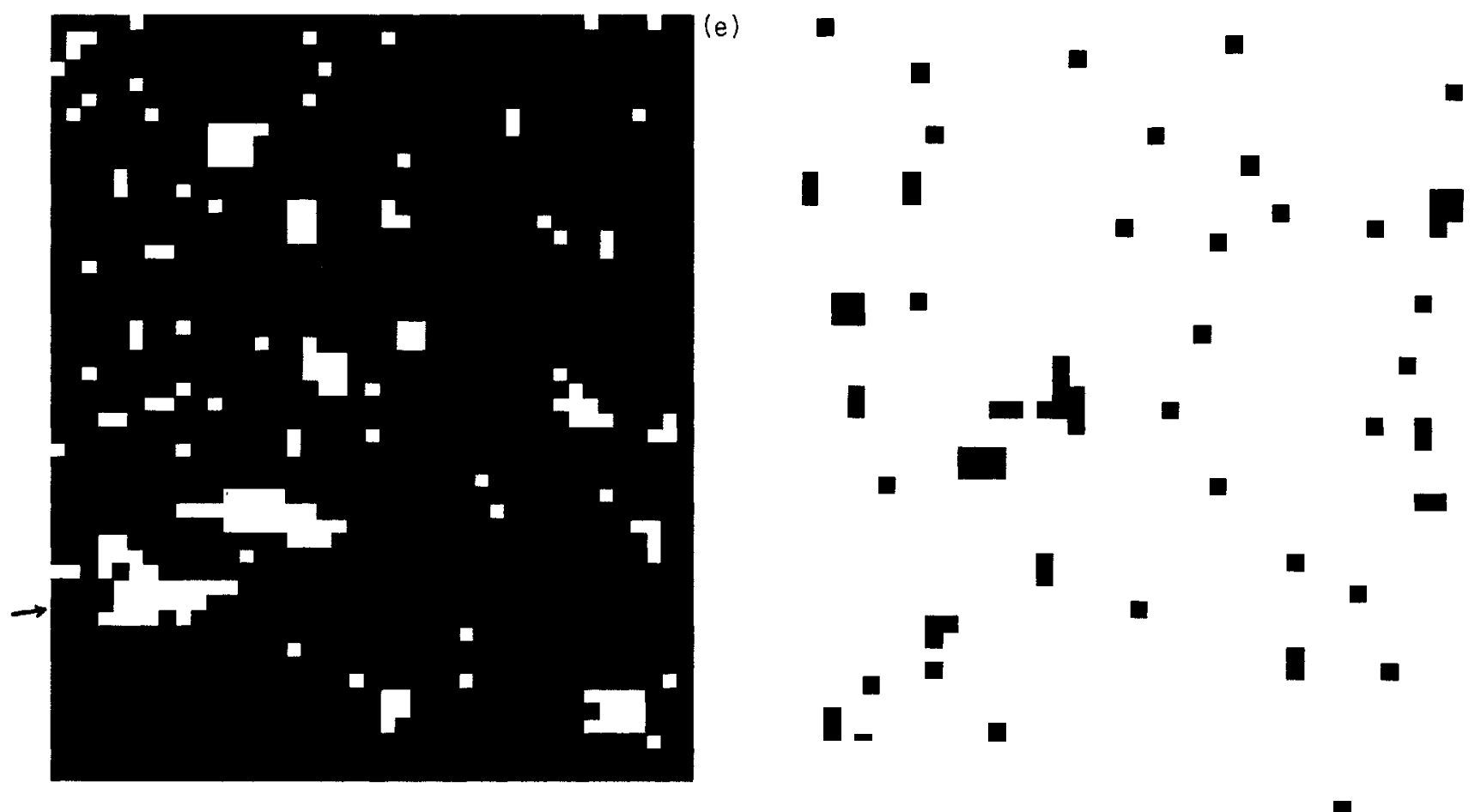

FIG. 35, (Continued).

theories thus predict a large value for $H_{c}$ equal to the spinodal field.

The theory of Shenoy and Agarwal ${ }^{16}$ contains enough dynamical features to be able to address the dependence of the shapes of hysteresis loops on the frequency of the applied field. They do not analyze this frequency dependence in the way we do (Sec. II); instead, they determine the range of frequencies, which they call a hysteresis window, in which conventional loops are obtained, as opposed to simple jumps in the order parameter or no jump at all. These calculations neglect spatial fluctuations of the order parameter which are present in many-body systems like magnets. Moreover, as the field changes, the free-energy curve changes and hence the extrema, the curvatures at the extrema, and the barrier height vary as functions of time; these effects are not incorporated in the calculation of the hysteresis window.

All theories mentioned above (with the exception of the Shenoy-Agarwal theory) treat hysteresis using equilibrium notions. Since, in a typical hysteresis experiment, the magnetic field is varied with a frequency of (say) 50 $\mathbf{H z}$, any equilibrium (static) description of hysteresis is strictly invalid. In this paper we have attempted to construct a nonequilibrium statistical-mechanical theory of hysteresis wherein thermal fluctuations (which are spatially modulated) of the order parameter are incorporated. To the best of our knowledge, this has not been attempted so far, nor has there been an attempt to understand the power-law behavior of the area of the hysteresis loop (e.g., the Steinmetz law) from a microscopic or a statistical mechanical point of view and to elucidate, if possible, any potential universality (in the renormalizationgroup sense) of this powerlaw. In this paper, we have at- tempted to systematize the study of some of the issues mentioned above for the two model spin systems considered in the text.

\section{EXPERIMENTAL RELEVANCE}

The two models we have studied in this paper are very simplified versions of models for real magnets. Thus, these models can clearly not describe all the phenomena that accompany the hysteretic behavior of magnets. Before discussing which phenomena our models might describe, we would like to emphasize that certain qualitative features of our results apply to real magnets: In particular, all magnets must show the regions $1-3$ of Fig. 11; if the formation of domains makes $M_{\text {eq }}=0$ in a magnet, then such a magnet will not show regions 4 and 5 (see below); in all magnets, a plot of the ratio $\mathrm{R}=|\tilde{\boldsymbol{M}}(3 \Omega)| /|\tilde{\boldsymbol{M}}(\Omega)|$ versus $H_{0}$ is qualitatively similar to Fig. 14; and hysteretic losses in a magnet must have a frequency dependence shown in Fig. 17 (this prediction of our theory has obvious and important implications for high-frequency magnetics).

The continuum model [Eq. (6)] that we study does not include the effects of magnetic anisotropy, dipolar forces, magnetoelastic couplings, and defects. All these can affect the hysteretic behavior of a magnet. For example, magnetic anisotropies and dipolar forces lead to the formation of domains in real magnets. On the application of a magnetic field, the walls between these domains move or the domains rotate; clearly, the effects of these phenomena on hysteresis loops cannot be accounted for in our model. ${ }^{27}$ Furthermore, in polydomain magnetic samples, the net zero-field equilibrium magnetization is zero. 
Thus, these samples should not exhibit loops of types 4 and 5 [Figs. 10(d) and 10(e)]. However, as $\Omega$ increases at fixed $H$, these samples should exhibit narrower and narrower loops of type 3 [Fig. 10(c)] since $M_{\text {in }}=M_{\text {eq }}=0$. Finally, our model can clearly not account for eddy-current losses in conducting magnets. Thus, the results that we obtain for our continuum model [Eq. (6)] should be of direct relevance to small, insulating, defect-free, singledomain particles with a very small magnetic anisotropy, and a low magnetoelastic coupling.

Insulating ferromagnets are often adequately described by the Heisenberg model. ${ }^{28}$ Our model is a continuum version of the Heisenberg model and allows the order parameter @ to have $N$ components. In many magnets $N=3$, but, for calculational convenience, we study the limit $N=\infty$ in which the transverse correlation functions dominate the longitudinal ones. (We expect that the results we obtain in this limit are qualitatively similar to those that we should obtain with $N=3$.) The resulting free-energy functional [Eq. (6)] is $O(N)$ symmetric and can be expanded in a polynomial in $\Phi^{2}$. For simplicity we restrict ourselves to the study of a model in which this polynomial is truncated beyond the $\left(\boldsymbol{\Phi}^{2}\right)^{2}$ term [we consider a $\left(\boldsymbol{\Phi}^{2}\right)^{3}$ theory in a subsequent paper]. Equilibrium thermodynamic functions and correlation functions can be calculated via a systematic $1 / N$ expansion in any dimension $\boldsymbol{d} .{ }^{29}$

The components of $\Phi$ evolve in time because of the time-varying magnetic field and thermal fluctuations. We have mimicked the effects of thermal fluctuations by a noise term in a Langevin equation [Eq. (3)]. This noise term represents the effect of a heat bath which, in insulating ferromagnets, is the lattice. Thus, the relaxation of the order parameters to equilibrium is accomplished by the excitation of phonons. We restrict ourselves to temporal variations of $\Phi$ that are much slower than phononrelaxation times. ${ }^{30}$ Thus, the components of $\Phi$ evolve purely relaxationally; ${ }^{31}$ the relaxation is accounted for by a phenomenological parameter, the kinetic coefficient $\Gamma$, which can be taken to be the spin-lattice relaxation time. The spin-lattice relaxation time can be obtained from ferromagnetic resonance linewidth measurements ${ }^{32}$ and is approximately $10^{-8} \mathrm{sec}$.

Equation (3) for the order parameters $\Phi_{\alpha}$ can be made plausible if $\Phi_{\alpha}$ relaxes slowly on the scale of $\Gamma^{-1}$ and if the phonons can actually be represented by a heat bath (i.e., the relaxation time of the phonons is much less than $\Gamma^{-1}$ ). The effects of the heat bath enter Eq. (3) in two ways - via, the kinetic coefficient and via the white noise $\eta_{\alpha}$

In general, $\Gamma$ is a function of the parameters in the theory, namely the temperature $r$, the coupling constant $u$, the strength of the magnetic field $H$, the frequency of the magnetic field $\Omega$, and the wave vector $q$. Since we consider a model in which no order parameters are conserved, $\Gamma(q=0) \neq 0$. In our analysis we assumed $\Gamma$ to be independent of $H_{0}$ and $\Omega$ and therefore, for given $r$ and $u$, the kinetic coefficient is a constant.

In our continuum model [Eq. (6)] $\Phi_{\alpha}$ is taken to be a nonconserved order parameter even when $H_{0}=0$. For a ferromagnet that is described by a Hamiltonian that is isotropic in spin space [like our model, Eq. (6)], the magnitude of the homogeneous magnetization vector is conserved. As soon as $H_{0}$ is nonzero, spin isotropy is broken and therefore $\Phi_{\alpha}$ will be a nonconserved order parameter. It might seem, therefore, that when $H_{0}$ is zero, the kinetic coefficient $\Gamma$ should be taken to be proportional to $q^{2}$. We argue, however, that the order parameter should be nonconserved even when $H_{0}=0$. The kinetic coefficient $\Gamma$ can be expanded in powers of $q^{2}$, i.e., $\Gamma(q)=\Gamma_{0}+q^{2} \Gamma_{2}-\ldots$. When spin-lattice couplings are present $\Gamma_{0}$ is nonzero even for $H_{0}=0$. This implies that the magnetization is not conserved. We therefore take $\Gamma$ to be the inverse spin-lattice relaxation time. This is obtained experimentally from measurements of ferromagnetic resonance linewidths, $\boldsymbol{A} \boldsymbol{H}$. The spin-lattice relaxation times can be extracted from the linewidths (which are typically $\approx 100 G$. This gives a spin-lattice relaxation time of around $10^{-8} \mathrm{sec}$. $\Gamma$ depends on $H, \Omega$, and $r$. For insulating magnets with a low magnetoelastic coupling, has a very weak dependence on $H_{0}, \Omega$, and $r$. In our analysis the temperature is held fixed while the magnetic field is changed, so the only serious assumption is the weak dependence of $\Gamma$ on the magnetic field.

The order parameter $\Phi_{\alpha}$ evolves purely relaxationally via the Langevin equation (3). A more general dynamical equation would involve a kinetic term given by the Poisson bracket $\left\{\Phi_{\alpha}, \Phi_{\beta}\right\}$. Such a term does not have a natural microscopic representation when $N>3$. In the study of critical dynamics this term corresponds to a relevant parameter, ${ }^{33}$ it would therefore be important to study the effect of this term on the hysteretic behavior of the $N=3$, $\left(\boldsymbol{\Phi}^{2}\right)^{2}$ model.

In spite of the limitations noted above, the qualitative results of our study (see above) must be valid for a wide variety of materials. Our results make it clear that there is a lot of interesting physics in the phenomenon of hysteresis that is worthy of experimental and theoretical study. In particular, it would be of great interest to study the scaling behavior (with respect to $H_{0}$ and $\Omega$ ) of the area of the hysteresis loop and the universality classes that may govern this.

We expect our results for Ising ferromagnets to be of relevance to hysteresis in strongly uniaxial ferromagnetic thin films or layered compounds (e.g., $\left.\mathrm{FeCl}_{2}\right)$. The magnetic structure of $\mathrm{FeCl}_{2}$ consists of ferromagnetic laye $\mathrm{r} \sim$ with an intralayer exchange coupling $J$ and a much weaker antiferromagnetic interlayer coupling $J^{\prime}$. Such ferromagnets show sharper, squarish hysteresis loops in contrast to the spindle-shaped hysteresis loops of isotropic magnets. However, we add a word of caution: Monte Carlo dynamics can only roughly mimic the dynamics of real magnets, since we neglect several features present in laboratory magnets. In particular, we neglect dipolar forces (demagnetization fields) which are especially important for finite-size systems. ${ }^{35}$ Moreover, we neglect the coupling of the constituent magnetic moments to the lattice. Spin-wave fluctuations are the dominant mechanism for the decay of the magnetization from a metastable state to a stable one in our continuum model 
[Eq. (6)]. By contrast, an activated process is required for such a decay in the Ising model. ${ }^{36}$ However, it is worth noting that the systematics of the evolution of the shapes of hysteresis loops (with the amplitude and frequency of the field) are similar in our continuum model [Eq. (6)] and the Ising model. As we have stated above with certain provisos, such evolution should also obtain in real magnets.

We have attempted to construct a nonequilibrium statistical-mechanical theory of hysteresis in two model spin systems. We have included the effect of thermally generated spatial fluctuations of the order parameter on hysteretic behavior. In this paper we have analyzed the dependence of shapes and areas of the hysteresis loops on the frequency and amplitude of the applied field and on temperature.

\section{ACKNOWLEDGMENTS}

It is a pleasure to thank Deepak Dhar, and the Theory Physics Group, Indian Institute of Science, for several beneficial discussions. This work was funded by the Council of Scientific and Industrial Research, India, the

University Grants Commission, India, and the Department of Science and Technology, India.

\section{APPENDIX: FORMAL RESULT DERIVED FROM A LANGEVIN EQUATION}

In this appendix we show that under certain conditions, a homogeneous initial magnetization remains homogeneous at all times when the spin system is subject to a time-varying magnetic field. We define a local free-

energy functional to be a free-energy functional which contains only local terms in the order parameter $\Phi_{\alpha}$ and its derivatives. The model free-energy functional Eq. (6) is of this form. A dynamical model in which the order parameter follows a Langevin equation (3) where $\beta F$ is a local free-energy functional will be called a local Langevin model. The local Langevin model $(\mathcal{L}, F,\{\Phi\})$ thus consists of a Langevin equation, $\mathcal{L}$, a local freeenergy functional, $\boldsymbol{F}$, and an $\mathrm{N}$-component order parame- ter $\left\{\Phi_{\alpha}\right\}$. The initial data required to solve the Langevin equation are all the n-point averages of $\Phi_{\alpha}$ (the averages are taken over the Gaussian white noise).

\section{Result}

Consider a local Langevin model $(\mathcal{L}, F,\{\boldsymbol{\Phi}\})$. If the initial conditions prescribed for $\mathcal{L}$ are such that

$$
\left\langle\prod_{\alpha i} \Phi_{\alpha}\left(\mathbf{q}_{i}, 0\right)\right\rangle=\left\langle\prod_{\alpha i} \Phi_{\alpha}\left(\mathbf{q}_{i}\right)\right\rangle_{0} \delta\left(\sum_{i} \mathbf{q}_{i}\right)
$$

where \langle\rangle$_{0}$ is the equilibrium average over the probability distribution $e^{-\beta F}$, then at subsequent times $\left\langle\prod_{\alpha i} \Phi_{\alpha}\left(q_{i}, t\right)\right\rangle$ is such that

$$
\sum_{i} q_{i}=0
$$

\section{Proof}

To be specific let us consider the free-energy functional given by Eq. (6). It will be obvious that the result stated above does not depend on the particular form of $F$ as long as it is local. The Langevin equation with $\boldsymbol{F}$ is given by

$$
\begin{aligned}
& \frac{d \Phi_{\alpha}(\mathbf{q}, t)}{\mathbf{d t}}=-\Gamma\left[q^{2} \Phi_{\alpha}(\mathbf{q}, t)+r \Phi_{\alpha}(\mathbf{q}, t)-H_{\alpha}(t) \delta_{\mathrm{q}, 0}\right. \\
&+ u \Phi_{\alpha}\left(\mathbf{q}-\mathbf{q}^{\prime}\right) \Phi_{\beta}\left(\mathbf{q}^{\prime \prime}, \mathbf{t}\right) \\
&\left.\times \Phi_{\beta}\left(\mathbf{q}^{\prime}-\mathbf{q}^{\prime \prime}, t\right)\right]+\eta_{\alpha}(\mathbf{q}, t)
\end{aligned}
$$

Repeated indices are summed (integrated) over. The equations for the moments lead to an infinite hierarchy of coupled equations. Consider the equation for the $n$-point correlation function $\left\langle\prod_{i} \Phi_{\alpha i}\left(\mathbf{q}_{i}, t\right)\right\rangle$. We note that

$$
\frac{d}{\mathbf{d t}}\left\langle\prod_{i} \Phi_{\alpha_{i}}\left(\mathbf{q}_{i}, t\right)\right\rangle=\sum_{i}\left\langle\frac{\bar{d}}{d t} \Phi_{\alpha_{i}}\left(\mathbf{q}_{i}, t\right) \prod_{j \neq i} \Phi_{\alpha_{j}}\left(\mathbf{q}_{j}, t\right)\right\rangle
$$

Therefore,

$$
\begin{aligned}
\frac{d}{d t}\left\langle\prod_{i} \Phi_{\alpha_{i}}\left(\mathbf{q}_{i}, t\right)\right\rangle=\sum_{i}[ & -\Gamma_{i}\left\{q_{i}^{2}\left\langle\prod_{j} \Phi_{\alpha_{j}}\left(\mathbf{q}_{j}, t\right)\right\rangle+r\left\langle\prod_{j} \Phi_{\alpha_{j}}\left(\mathbf{q}_{j}, t\right)\right\rangle-D \delta_{\alpha_{i^{\prime}} 3}\left\langle\Phi_{\alpha_{i}} \cdots \Phi_{\alpha_{n}}\right\rangle\right. \\
& -H_{\alpha_{i}}(t) \delta_{q_{i}} 0\left\langle\prod_{j \neq i} \Phi_{\alpha_{j}}\left(\mathbf{q}_{j}, t\right)\right\rangle \\
& \left.+u\left\langle\Phi_{\alpha_{i}}\left(\mathbf{q}_{1}\right) \cdots \Phi_{\alpha_{i}}\left(\mathbf{q}_{i}-\mathbf{q}^{\prime}\right) \Phi_{\beta}\left(\mathbf{q}^{\prime}-\mathbf{q}^{\prime \prime}\right) \Phi_{\beta}\left(\mathbf{q}^{\prime \prime}\right) \cdots \Phi_{\alpha_{n}}\left(\mathbf{q}_{n}\right)\right\rangle\right] \\
& \left.+\left\langle\Phi_{\alpha_{1}}\left(\mathbf{q}_{1}\right) \cdots n_{\alpha_{i}}\left(\mathbf{q}_{i}\right) \cdots \Phi_{\alpha_{n}}\left(\mathbf{q}_{n}\right)\right\rangle\right] .
\end{aligned}
$$

The differential equation can be treated as an iterative map which transforms the n-point correlation function at time $\mathrm{t}$ to $t+1$. Thus, if at time $t=0$, each term on the right-hand side of Eq. (A5) is proportional to $\delta\left(\Sigma_{j} q_{j}\right)$, then the n-point correlation function at subsequent times will also be proportional to $\delta\left(\boldsymbol{\Sigma}_{j} \mathbf{q}_{j}\right)$ (since the independent variable of the differential equation is $t$ ). Therefore, all we are required to show is that each term on the 
right-hand side of Eq. (A5) is proportional to $\delta\left(\Sigma_{j} \mathbf{q}_{j}\right)$.

It is obvious that the first two terms are proportional to $\delta\left(\Sigma_{j} \mathbf{q}_{j}\right)$. The fourth term is an $(n-1)$-point correlation function. At $t=0$.

$$
\left\langle\prod_{j \neq i} \Phi_{\alpha_{j}}\left(\mathbf{q}_{j}\right)\right\rangle \sim \delta\left[\Sigma_{j}^{\prime} \mathbf{q}_{j}\right]
$$

where $\Sigma^{\prime}$ indicates that the ith term is dropped from the sum. Thus, at $t=0$,

$$
H_{\alpha_{i}}(t) \delta_{\mathbf{q}_{i} 0}\left\langle\prod_{\substack{j \neq i \\ j \neq i}} \Phi_{\alpha_{j}}\left(\mathbf{q}_{j}\right)\right) \sim \delta\left(\boldsymbol{\Sigma}_{j} \mathbf{q}_{j}\right) .
$$

The third term $u\left\langle\Phi_{\alpha_{i}} \cdots \cdot \Phi_{\alpha} \Phi_{\beta} \Phi_{\beta} \cdots \Phi_{\alpha}\right\rangle$ is an $(n+2)$-point correlation function, which at $t=0$ is proportional to

This is equal to $\delta\left(\Sigma_{j} \mathbf{q}_{j}\right)$. The last term

$$
\left\langle\Phi_{\alpha_{i}}\left(\mathbf{q}_{1}\right) \ldots \eta_{\alpha_{i}}\left(\mathbf{q}_{i}\right) \ldots \Phi_{\alpha_{n}}\left(\mathbf{q}_{n}\right)\right\rangle
$$

is also proportional to $\delta\left(\Sigma_{j} \mathbf{q}_{j}\right)$. This can be seen by appealing to Novikov's theorem, ${ }^{37}$ which states that for a Gaussian random noise $\eta_{\alpha}$

$$
\begin{aligned}
\left\langle\eta_{\alpha} f(\{\boldsymbol{\Phi}\})\right\rangle & =\sum_{\beta} \int\left\langle\eta_{\alpha}\left(\mathbf{q}_{i}\right) \eta_{\beta}\left(\mathbf{q}^{\prime}\right)\right\rangle\left\langle\delta f / \delta \Phi_{\beta}\right\rangle_{\mathbf{q}=\mathbf{q}^{\prime}} d \mathbf{q}^{\prime} \\
& =\sum_{\beta} 2 \Gamma \int \delta_{\alpha_{\beta} \beta} \delta\left(\mathbf{q}_{i}+\mathbf{q}^{\prime}\right)\left\langle\delta f / \delta \Phi_{\beta}\right\rangle_{\mathbf{q}=\mathbf{q}^{\prime}} d \mathbf{q}^{\prime} .
\end{aligned}
$$

We are interested in an $f$ given by

$$
f(\{\Phi\})=\prod_{j \neq l} \Phi_{\alpha_{j}}\left(\mathbf{q}_{j}\right) \text {. }
$$

After some algebra and an integration over $\mathbf{q}^{\prime}$ we get,

$$
\begin{aligned}
& \left\langle\eta_{\alpha_{i}}\left(\mathbf{q}_{i}\right) f(\{\boldsymbol{\Phi}\})\right\rangle \\
& \quad=\sum_{m} \delta_{i, m} \delta\left(\mathbf{q}_{i}+\mathbf{q}_{k}\right) \sum_{k \neq i}\left\langle\prod_{j \neq i, k} \Phi_{\alpha_{j}}\left(\mathbf{q}_{j}\right)\right) \delta_{k, m} .
\end{aligned}
$$

At $t=0$

$$
\left\langle\sum_{j \neq i, k} \Phi_{\alpha_{j}},\left(\mathbf{q}_{j}\right)\right\rangle \sim \delta\left(\sum_{j \neq i, k} \mathbf{q}_{j}\right)
$$

This, together with the $\delta\left(\mathbf{q}_{i}+\mathbf{q}_{k}\right)$ term in Eq. (A8), implies that

$$
\left\langle\eta_{\alpha_{i}}\left(\mathbf{q}_{i}\right) f(\{\boldsymbol{\Phi}\})\right\rangle \sim \delta\left(\sum_{j} \mathbf{q}_{j}\right) .
$$

Thus, all terms on the right-hand side of Eq. (A5) at $t=0$ are proportional to $\delta\left(\Sigma_{j} \mathbf{q}_{j}\right)$. Let us analyze this result in some detail. The moments at time $t=0$ are assumed to be given by their equilibrium values at $H=0$. Since there are no dipolar forces, this implies that the initial magnetization is homogeneous. As the field changes one might expect the magnetization to become inhomogeneous. One would then have to deal with a magnetization which is $q$ dependent. The above result, however, states that given an initial homogeneous spin configuration, inhomogeneities do not develop at later times. It can be seen that the inclusion of nonlocal interactions in the freeenergy functional would produce inhomogeneous spin configurations, even though the initial spin configuration is homogeneous. In particular, the inclusion of dipolar interactions produces inhomogeneities in the magnetization. We thus we see that, within our model, magnetization reversals occur via spatially homogeneous spin configurations only. Strictly speaking, the above derivation assumes that translational invariance is not spontaneously broken and that the correlation functions do not acquire anomalous q-dependent expectation values. ${ }^{38}$
*Present address: Department of Physics, Simon Fraser University, Burnaby, British Columbia, Canada V5A 1 S6.

1 J. D. Gunton, M. San Miguel, and P. S. Sahni, in Phase Transitions and Critical Phenomena, edited by C. Domb and J. L. Lebowitz (Academic, London, 1983), Vol. 8.

${ }^{2}$ G. S. Grest, M. P. Anderson, and D. J. Srolovitz (unpublished).

${ }^{3}$ R. M. Bozorth, Ferromagnetism (Van Nostrand, New York, 1951).

${ }^{4}$ J. Smit and H. P. J. Wijn, Ferrites (Wiley, New York, 1959).

5J. A. Ewing and H. G. Klaassen, Philos. Trans. R. Soc. London, Ser. A 184A, 985 (1893).

${ }^{6} \mathrm{~J}$. A. Ewing, Magnetic Induction in Iron and Other Metals (The Electrician, London, 1900).

${ }^{7}$ C. P. Steinmetz, Trans. Am. Int. Electr. Eng. 9, 3 (1892).

${ }^{8}$ L. Landau and E. M. Lifshitz, Electrodynamics of Continuous Media (Pergamon, New York, 1984), Vol. 8.

${ }^{9}$ G. Mazenko and M. Zannetti, Phys. Rev. B 32,4565 (1985).

${ }^{10} \mathrm{M}$. Rao, Ph.D. thesis, Indian Institute of Science, Bangalore, India, 1988.

${ }^{11}$ A. Z. Patashinski and V.L. Pokrovskii, Fluctuating Theory of Phase Transitions (Pergamon, New York, 1979).

${ }^{12}$ W. H. Press, B. P. Flannery, S. A. Teukolsky, and W. T. Vetterling, Numerical Recipes (Cambridge University Press,
Cambridge, 1986), Chap. 15.

${ }^{13}$ In our model the saturation magnetization of the hysteresis loop increases monotonically with $H_{0}$. Loops of type 1 do not saturate at a fixed value of the magnetization. This is because, in our model, $\Phi_{c}$ is not a fixed-length spin.

${ }^{14}$ Hysteresis loops which have the symmetry of $M \rightarrow-M$ and $\boldsymbol{H} \rightarrow-H$ will be called symmetric hysteresis loops.

${ }^{15}$ Strictly speaking, linear response is invalid but it should be a fair approximation when the magnetization is close to zero.

${ }^{16}$ G. S. Agarwal and S. R. Shenoy, Phys. Rev. A 23,2719 (1981); S. R. Shenoy and G. S. Agarwal, ibid. 29, 1315 (1984).

${ }^{17}$ N. Metropolis, M. N. Rosenbluth, A. Rosenbluth, A. Teller, and E. Teller, J. Chem. Phys. 21, 1087 (1953).

${ }^{18} \mathrm{~K}$. Binder, in Monte Carlo Methods in Statistical Physics, edited by K. Binder (Springer, Berlin, 1979), Chap. 1.

${ }^{19}$ B. M. McCoy and T. T. Wu, Two-Dimensional Ising Model (Harvard University Press, Cambridge, 1973).

${ }^{20}$ The noise that is seen in Fig. 28(a) is because of the Monte Carlo dynamics. The standard deviation at any point on the curve is of the order of $10^{-4}$.

${ }^{21}$ Our results might be consistent with the following scaling relations (as conjectured in Ref. 10): $H_{c} \approx \Omega^{\mu} \Upsilon\left(N^{\gamma} \Omega\right)$, $H_{c} \approx \Omega^{\varepsilon} \Psi\left(T^{\eta} / \Omega\right)$, and a similar one for the area of the loop. 
(In the previous relations, variables not specified are held fixed.) The behavior of hysteresis loops with respect to the frequency and the amplitude of the field suggests that a scaling relation of the form for the area $A \approx \Omega^{\alpha}\left(H_{0}^{\beta} / \Omega\right)(N$ and $T$ constant) might exist. This is in conformity with the scaling relation observed in the $\left(\Phi^{2}\right)^{2}$ model. Our simulation studies are not extensive enough to test these conjectures. The validity of these scaling relations has to be tested by performing long simulations on larger lattices (this is being carried out now).

${ }^{22}$ J. S. W. Rayleigh, Philos. Mag. 23, 225 (1887).

${ }^{23}$ E. Preisach, Z. Phys. 94,277 (1935).

${ }^{24}$ B. D. Coleman and M. L. Hodgdon, Int. J. Eng. Sci. 24, 897 (1986);M. L. Hodgdon, IEEE Trans. Magn. 24,218 (1988);B. D. Coleman and M. L. Hodgdon, Arch. Rational Mech. Anal. 99, 375 (1987).

${ }^{25}$ E. C. Stoner, Phys. Soc. Rep. Prog. Phys. 13, 83 (1950).

${ }^{26}$ A. H. Morrish, The Physical Properties of Magnetism (Wiley, New York, 1965).

${ }^{27}$ We have initiated a study in which the effects of anisotropy are studied. There exists no simple $1 / N$ expansion if we include an anisotropy of the form $D \Sigma_{i}\left(S_{i}^{2}\right)^{2}$; however, simple closure approximations yield hysteresis loops similar to those obtained here: as the anisotropy increases, the loops become squarish [M. Rao et al. (unpublished)].

${ }^{28} \mathrm{C}$. Herring, in Magnetism, edited by G. T. Rado and H. Suhl (Academic, New York, 1966), Vol. II B.

${ }^{29}$ S. K. Ma, in Phase Transitions and Critical Phenomena, edited by C. Domb and M. S. Green (Academic, New York, 1977), Vol. 6.

${ }^{30}$ The phonons can be represented by a heat bath since the phonon-relaxation time is much smaller than the time scale of variation of the order parameter. This implies that the spin temperature is the same as the lattice temperature. The phonon relaxation time goes as $\left(\Theta_{D} / T\right)^{5}$ [see A. Akhiezer, V. Bar'yakhtar, and S. Peletminskii, Spin Waves (NorthHolland, Amsterdam, 1968)], where $\Theta_{D}$ and Tare the Debye and the phonon heat bath temperature, respectively. At low temperatures, phonons relax slowly and therefore do not represent a good heat bath.

${ }^{31}$ Strictly speaking, for a Heisenberg ferromagnet, isotropy in spin space implies that the magnetization is conserved. Whereas this conservation law may be of relevance in some physical situations, we restrict ourselves to cases where the coupling of the spins to the lattice is sufficient to lead to the relaxational dynamics we use. Effects due to domain rotation and growth are not considered in our model.

${ }^{32}$ N. Bloembergen and S. Wang, Phys. Rev. 93, 72 (1954).

${ }^{33}$ S. K. Ma, Modern Theory of Critical Phenomena (Benjamin, Reading, Mass., 1976).

${ }^{34}$ L. de Jongh and A. Miedema, Adv. Phys. 23, 1 (1974).

${ }^{35}$ The demagnetization factor in our simulations is zero. This is because of our use of periodic boundary conditions which makes the finite square spin sample topologically equivalent to a torus. A torus has a zero demagnetization factor.

${ }^{36}$ The relaxation time of the magnetization can be derived from simple nucleation theory arguments and goes as $\exp \left(1 / H_{0}\right)$ in the case of the Ising model. As seen in Sec. II F, the relaxation time for the continuum model [Eq. (6)] goes as $H_{0}^{-1}$.

${ }^{37}$ E. A. Novikov, Zh. Eksp. Teor. Fiz. 47, 1919 (1964) [Sov. Phys. - JETP 20, 1290(1965)].

${ }^{38}$ We thank D. Dhar for pointing this out to us. 

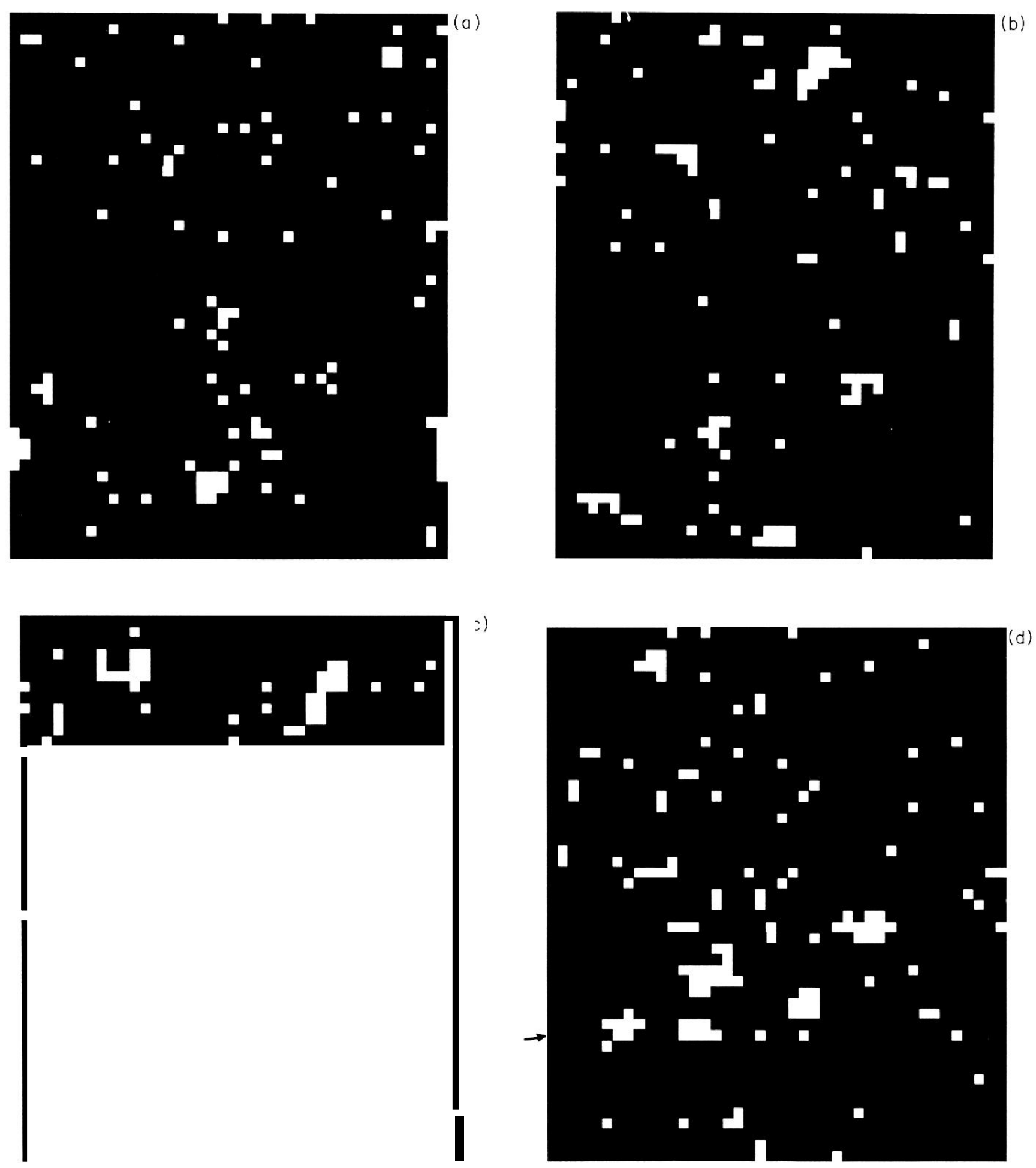

FIG. 35. Time sequence of spin configurationson a $50 \times 50$ lattice as a type-I hysteresis loop is traversed from $H=-H_{0}=-2$ to $H=H_{0}=2$. Solid squares denote $S_{i}=-1$. Note that even when $H>0$, the spins are predominantly "down." (a) $H=-0.08$, (b) $H=-0.02$, (c) $H=0.02$, (d) $H=0.04$, (e) $H=0.08$, and (f) $H=0.16$. The cluster, marked by an arrow in (d) and (e), shows coarsening and coalescence. 

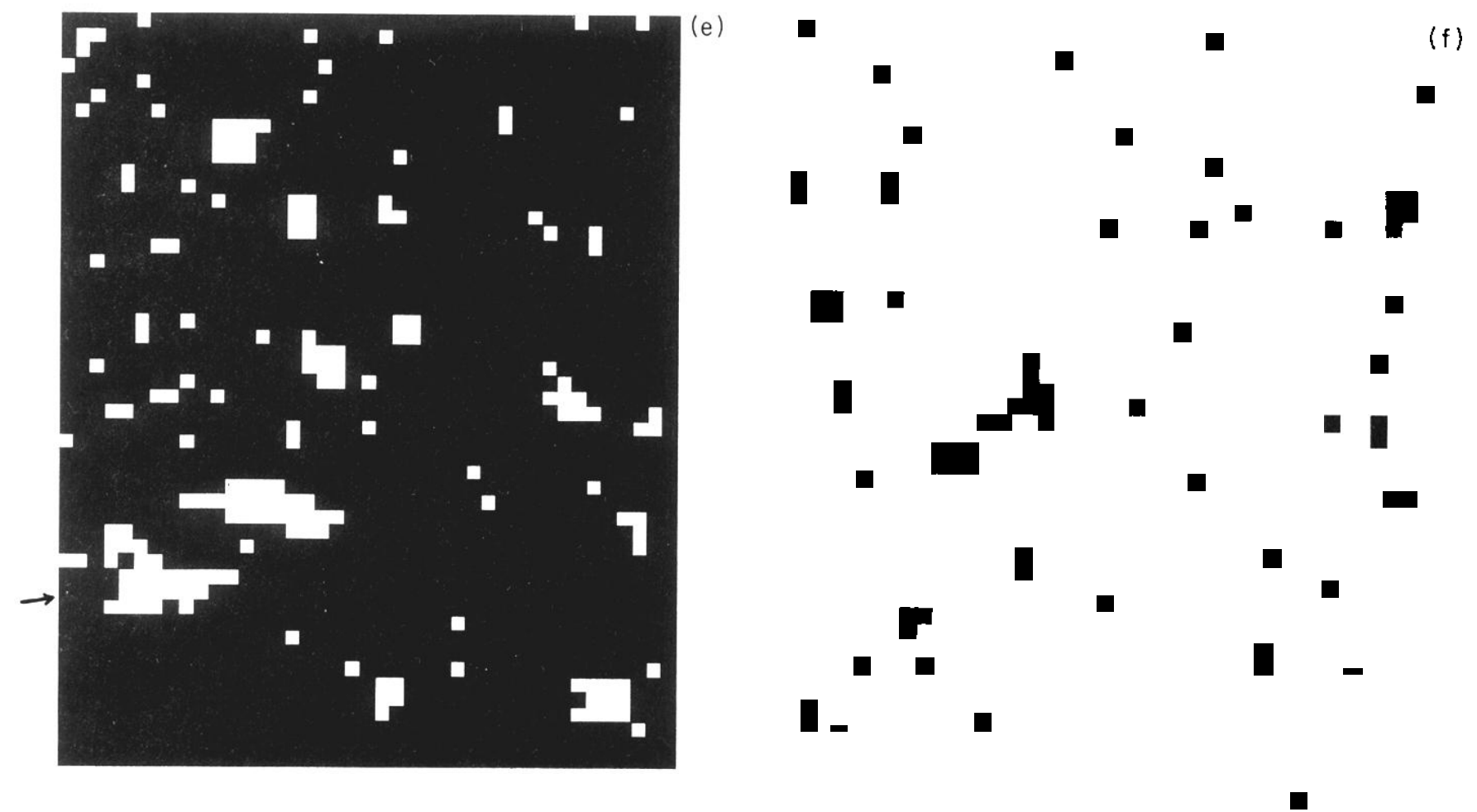

FIG. 35. (Continued) 